Omega-P, Inc.

Final Report on I DoE SBIR grant DE-SC000 4497

MODIFIED MAGNICON FOR HIGH-GRADIENT ACCELERATOR R\&D

Omega-P, Inc.

291 Whitney Avenue, Suite 401

New Haven, CT 06511

Final Report to US Department of Energy

SBIR Phase I Grant DE-SC000 4497

\title{
MODIFIED MAGNICON FOR HIGH-GRADIENT ACCELERATOR R\&D
}

\section{CONTENTS}

I. INTRODUCTION

p. 2

II. TECHNICAL APPROACH

IIa. Modified magnicon: conceptual approach, design overview and performance

IIb. New output cavity

IIc. Modifications to the system of deflection cavities

IId. Modifications to the magnetic system

IIe. Modifications to collector

IIf. Decision making process when designing and optimizing the output cavity

References

\section{SUMMARY}

Analysis, and low-power cold tests are described on a modified design intended for the Ka-band pulsed magnicon now in use for high-gradient accelerator R\&D and rare elementary particle searches at the Yale University Beam Physics Laboratory. The modification is mainly to the output cavity of the magnicon, which presently operates in the $\mathrm{TM}_{310}$ mode. It is proposed to substitute for this a dual-chamber $\mathrm{TE}_{311}$ cavity structure. The first chamber is to extract about $40 \%$ of the beam power (about $25 \mathrm{MW}$ ) at $34.272 \mathrm{GHz}$, while the second chamber is to convey the power to four WR-28 output waveguides. Minor design changes are also proposed for the penultimate $11.424 \mathrm{GHz}$ cavity and the beam collector. The intention of these design changes is to allow the magnicon to operate reliably $24 / 7$, with minor sensitivity to operating parameters.

Work on this project was carried out by senior research consultant Dr. V.P. Yakovlev of FNAL and research scientist Dr. S.V. Shchelkunov of Yale University, with Dr. J.L. Hirshfield serving as Principal Investigator. The report was edited by Dr. Hirshfield.

December 19, 2011 


\section{Omega-P, Inc. \\ Final Report on I DoE SBIR grant DE-SC000 4497 \\ MODIFIED MAGNICON FOR HIGH-GRADIENT ACCELERATOR R\&D}

\section{INTRODUCTION}

Under Topic 65c in the 2010 SBIR Program Solicitation, Phase I grant applications were sought by DoE to develop RF sources for high-gradient accelerator R\&D. In response, OmegaP, Inc. submitted a Phase I proposal and was awarded grant DE-SC000 4497, entitled "Modified Magnicon for High-Gradient Accelerator R\&D.” The principal Phase I goal was to design a new output cavity for the eight-year old Omega-P/Yale Ka-band $(34.3 \mathrm{GHz})$ magnicon amplifier. Incorporating a new output cavity ( $\mathrm{TE}_{311}$ mode in place of $\mathrm{TM}_{310}$ mode) is predicted to improve the tube's performance for high-gradient accelerator $R \& D$ experiments, and to increase the chances of new discoveries in an ongoing laboratory search for weakly-interacting light bosons and axions. The Phase II goals were to engineer and build the new output cavity; install it in a brazed assembly with a new set of (original design) gain cavities and a new beam collector; and then bake, condition, and re-commission the tube. It was predicted that the modified tube will deliver a peak power at $34.3 \mathrm{GHz}$ of about $25 \mathrm{MW}$ in $1-\mu$ s pulses at a $10-\mathrm{Hz}$ rate. However, the Phase II portion of the project did not receive DoE approval.

The $34.3 \mathrm{GHz}$ magnicon, developed by Omega-P beginning in 1996, is the key element at the Yale Ka-Band Accelerator Test Facility.* As presently configured, the tube can supply limited-but diminishing-RF power for a number of current accelerator R\&D and particle physics experiments that will be described below. At this writing, the magnicon is able to deliver less than $4 \mathrm{MW}$ to loads connected to the four outputs of the tube. This is much lower than the 18 MW reported when the tube was first commissioned and even lower than the 40 MW predicted by simulations upon which the tube's design was first based. Explanations for these discrepancies are now understood to originate with the design itself, with the processing regimen to which the tube was subjected, and probably to unavoidable internal damage during its eight years of operation. Such design flaws and long-term degradation in a first-of-its kind prototype high-power mm-wave amplifier are probably not unprecedented; fortunately, a clear prescription that promises to ameliorate the magnicon's ills has been devised, as described in this document.

There are strongly compelling reasons to rebuild the $34.3 \mathrm{GHz}$ magnicon. The Yale University Beam Physics Lab was awarded grant support from the DoE Office of High Energy Physics in 2007 to establish and operate a Ka-Band Test Facility built around the magnicon; an extension of this grant is now scheduled to run at least through 2012. The Facility has already

\footnotetext{
*Experience in operating the 34-GHz magnicon has already provided proof that a $500-\mathrm{kV}, 200$ A, 1-mm diameter electron beam can be reliably formed and stably transported through a $20-\mathrm{cm}$ long multi-cavity structure with 5-mm apertures. Also proven is that a beam deflection (i.e., magnicon) interaction can be excited and amplified to multi-MW levels at $11.4 \mathrm{GHz}$ in a set of gain cavities, and that coherent third-harmonic output at $34.3 \mathrm{GHz}$ can be generated in a highlyover-moded output cavity, with $>50 \mathrm{~dB}$ of gain to deliver multi-MW Ka-band power through WR-28 waveguides into four separate loads.
} 


\section{Omega-P, Inc. \\ Final Report on I DoE SBIR grant DE-SC000 4497 \\ MODIFIED MAGNICON FOR HIGH-GRADIENT ACCELERATOR R\&D}

hosted a variety of experiments aimed at making systematic progress in high-gradient and particle physics research, and demand for use of this unique facility is increasing. Current projects include a quasi-optical RF pulse compressor with a plasma-switched grating as its active element; measurements of surface breakdown on CVD diamond; comparison of RF breakdown statistics between copper and high work-function metals such as platinum; measurements of surface fatigue due to pulsed heating attending high pulsed RF magnetic fields for different metals; studies aimed at increasing RF breakdown thresholds by use of strongly-adherent thin coatings on cavity surfaces; tests of a short-period RF undulator for a $\mathrm{nm}$ wavelength SASE radiation source; and search for low energy (meV's) hidden sector bosons and axions. Finally, it can be expected that experience that would have been gained in designing, building, and operating a 25-MW mm-wave pulsed amplifier should inform the wider US and international high-power microwave tube community, where R\&D programs of the type proposed here are in increasingly scarce supply.

Improved performance for the modified $34.3 \mathrm{GHz}$ magnicon would be based on the robust design to be described below, greatly facilitated by many ancillary systems and components that have been added to the Yale Ka-band Test Facility since its inauguration four years ago. These include a helium re-liquification system for the magnicon's superconducting magnet to eliminate need for expensive >100 liter/week LHe deliveries required previously; a model PNA E8364C Agilent vector network analyzer with calibration kits ranging from $10 \mathrm{MHz}$ to $50 \mathrm{GHz}$; a new set of 13-mm diameter high-power Ka-band transmission components, including new magnicon barrier windows and associated mode converters; a further set of Kaband components and vacuum pumps to constitute a new transmission line that passes through a radiation shielding wall to a test station where hands-on experimentation can take place during magnicon operation; and a successful DoE SBIR Phase I design and approved Phase II construction by Diversified Technologies Inc. of a $500-\mathrm{kV}, 200$ A solid-state Marx-type modulator to replace the aging, unreliable line-type modulator that presently powers the Ka-band magnicon at Yale. Taken together, these items add considerable strength to the capabilities that could be anticipated from the modified magnicon for present and future experiments in highgradient accelerator R\&D and in searches for rare hidden sector light bosons and axions.

This report is organized as follows: Section II -- Technical Approach, contains the essential new technical material that underlies the failed Phase II project. Sub-Section IIa is perhaps the most important, and describes the conceptual approach and gives a design overview and anticipated performance of the new tube. It is shown there that the tube would be a robust device intended to perform $24 / 7$ as needed at a user-type facility. Among other important things, it is also described how the new output cavity would be integrated with a new chain of drive and deflection cavities - these would be almost identical to the original versions - and with a new beam collector. It should be noted that all cavities and the collector in a single brazed assembly were to be subjected to prolonged high-temperature bakeout. Sub-Section IIb provides details of the new $\mathrm{TE}_{311}$-mode based magnicon output cavity that is the most crucial component to achieve 


\section{Omega-P, Inc. \\ Final Report on I DoE SBIR grant DE-SC000 4497 \\ MODIFIED MAGNICON FOR HIGH-GRADIENT ACCELERATOR R\&D}

the desired performance and tube behavior [as described in Sub-Section IIa]. Sub-section IIc describes modifications to the cavity deflection system, and concentrates mostly on the last deflection cavity (\#6), modifications of which are important in order to achieve good efficiency. Sub-Section IId presents modifications to the magnetic system. Sub-Section IIe is dedicated to a new collector. Sub-Section IIf is to provide the full list of details that were worked through to design the new output cavity; this material has been transferred from Sub-Section IIb for a reader interested in all aspects of the design process, without clogging Sub-Section IIb with all the details. Finally, Sub-Section IIg gives details about the status of the present magnicon and associated test facility infrastructure.

\section{TECHNICAL APPROACH}

\section{IIa. Modified Magnicon: Conceptual Approach, Design Overview and Performance}

Conceptual Approach: In order to have a robust tube that can operate 24/7, with output power $\sim 25 \mathrm{MW}$ in Ka-band, the following principles are to be followed: (a) as with other tubes designed for high-power collider-related applications (e.g. klystrons), the modified magnicon is to be a fully brazed tube without ceramics (save for the gun insulator); (b) the design is to allow bake-out up to $600{ }^{\circ} \mathrm{C}$ so that successful conditioning in a reasonable time can be achieved as experience has shown at SLAC, KEK and BINP (and at industrial tube companies); and (c) an output cavity operating in the $\mathrm{TE}_{311}$ mode is to be used to allow a significant decrease in the RF fields on the cavity walls as compared to the presently-employed $\mathrm{TM}_{310}$ mode, and in addition a significant decrease in the guiding magnetic field. The design leads to a wider bandwidth than in the present tube, making its performances far less sensitive to operating parameters and settings which is critical during conditioning.

The aforesaid principles contrast dramatically with those observed for the present design. The Ka-band magnicon amplifier was the first (partially) successful attempt to reach multi-MW levels for any amplifier in the mm frequency domain. However, the choice of technology for the tube was not optimal, since it could be neither fully-brazed nor subjected to high temperature bake-outs. Yet, at the time it was a well justified approach because there was need to have as much diagnostic for this RF tube prototype as possible; this choice resulted in a detachable design that contained ceramics to insulate the beam collector. This strategy was based on the earlier successful design of the $7 \mathrm{GHz}$ magnicon, where design parameters were obtained only after an extremely long and meticulous conditioning procedure. However, as experience with the $11.4 \mathrm{GHz}$ Omega-P/NRL magnicon has also demonstrated, it is obvious now that at higher frequencies this detachable technology does not work well. Conditioning of the presently operating $34 \mathrm{GHz}$ magnicon was a great challenge, worsened by several facts such as: (a) low repetition rate of the available modulator, (b) high fields in the output cavity, (c) sensitivity to high-magnitude guiding magnetic field because of a long-period interaction in a long output cavity, and (d) a narrow pass-band that imposes a high degree of sensitivity to phenomena 


\section{Omega-P, Inc.}

Final Report on I DoE SBIR grant DE-SC000 4497

\section{MODIFIED MAGNICON FOR HIGH-GRADIENT ACCELERATOR R\&D}

(multipactoring, breakdowns, outgasing, reflections from the loads) that usually take place during conditioning. It is now conjectured that in the long process of conditioning the output cavity was fatally damaged, which results in the initial low output power, and in its gradual diminution over the past eight years. The new modified magnicon proposed here-in contrast to the old tube - is thus designed to be free of these undesirable features.

Design Overview: The modified design [see Fig. 1] embodies the same sub-systems as the present magnicon, namely (1) electron gun with pulse transformer (all inside an oil tank); (2) multi-coil superconducting solenoid with its liquid helium reservoir and re-liquification system; (3) RF cavity deflection cavity chain consisting of an X-band input cavity and five X-band deflection gain cavities; (4) a frequency-tripling Ka-band output cavity; (5) beam collector; (6) kW-level TWT driver (at $11.4 \mathrm{GHz}$ ); (7) diagnostics; and (8) supporting subsystems such as chilled water for cooling, radiation shielding and monitoring, and the pulsed 500-kV, 200-A linetype modulator to power the electron gun.

The electron beam originates at the $50 \mathrm{~mm}$ diameter gun cathode, is compressed to about $1 \mathrm{~mm}$ diameter by the virtue of electrostatic gun design and insertion into a guiding magnetic field provided by the solenoid, and travels down the chain of deflection cavities. The deflection system consists of one drive cavity, three "angle-gain" cavities, and two "penultimate" cavities. Inside this chain the e-beam is deflected along the radius by the rotating $\mathrm{TM}_{110}$-mode. The resulting scanning beam rotates at a frequency of $11.424 \mathrm{GHz}$ (the frequency of the drive signal), enters the output cavity and generates radiation at three times the drive frequency - at 34.272 $\mathrm{GHz}$ - in the first chamber of the output cavity exciting the $\mathrm{TE}_{311}$ mode. The second chamber of the output cavity is strongly coupled with the first and is built with four WR-28 output waveguides that are coupled with the cavity via magnetic components of the RF-field to extract the produced power.

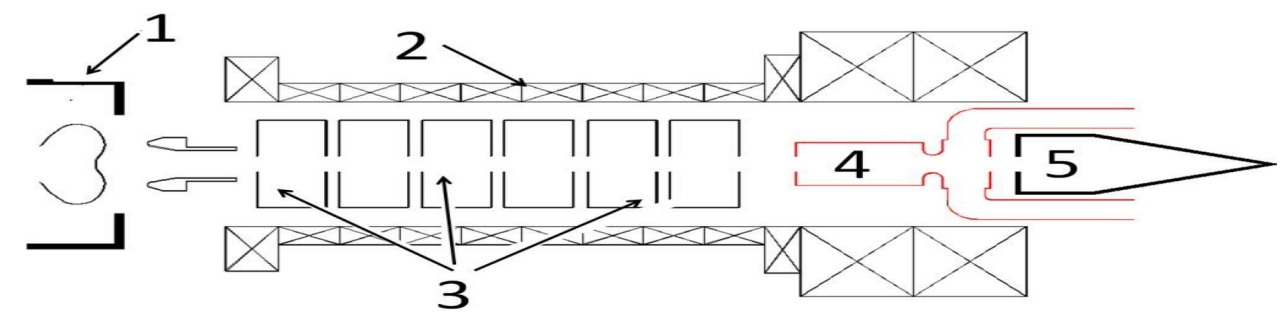

Fig. 1. Schematic of the tube (not to scale). 1 - electron gun; 2- superconducting solenoid; 3 - chain of six deflection cavities; 4 - two chamber output cavity with output waveguides; 5 - beam collector. Other components such as TWT driver, diagnostics system, etc. are not shown. Note that actual installation is vertical with the gun at the bottom.

It is instructive to review tube elements and their modifications (if any) following the electron beam path as it travels from the gun to collector. 


\section{Omega-P, Inc. \\ Final Report on I DoE SBIR grant DE-SC000 4497 \\ MODIFIED MAGNICON FOR HIGH-GRADIENT ACCELERATOR R\&D}

(1) Electron gun: It is planned to use the present gun, except that an available back-up cathode stem assembly will replace the existing one. The gun dynamics are well known, and its behavior is understood and the performance has been described in several publications [1,2]. Even though the gun was designed for $500 \mathrm{kV}$, it operates well in the range $460-475 \mathrm{kV}$ when the repetition rate is low; with high repetition rates (and especially with long pulses), the gun operates well at voltages at or below $450 \mathrm{kV}$. Consequently, the operating voltage for the modified tube is chosen to be $450 \mathrm{kV}$, and the modified magnicon is optimized for this voltage. This choice reduces the beam power from a maximum of $100 \mathrm{MW}$ to $84 \mathrm{MW}$.

(2) Superconducting multi-coil solenoid: The present solenoid can be used as is, to generate the static magnetic field profile suitable for the new tube. The magnetic field in the region of the deflection cavities varies from $\sim 11.870 \mathrm{kG}$ to $\sim 10.280 \mathrm{kG}$ and is provided by the coils fed with a current of $\sim 86 \mathrm{~A}$, which is well below the design limit of $100 \mathrm{~A}$. The magnetic field in the region of the output cavity varies from $\sim 10.280$ to $\sim 10.500 \mathrm{kG}$, and is provided by one bucking coil with a current $\sim 12 \mathrm{~A}$ and a coil triplet typically at a current $\sim 33 \mathrm{~A}$, which again is well below the allowed maximum of $100 \mathrm{~A}$. Because of the chosen operating mode (TE311) in the output cavity, as well as low loaded $Q$-factor and a relatively short length of the cavity, the generated power will be shown to be remarkably insensitive to changes in the current of the coil around the output cavity, a feature in a striking contrast to the present magnicon-tube with its extreme sensitivity to the correct tuning of coil currents. Some modifications to the magnetic system (coils, polepieces, iron insertions) were considered, but found unnecessary. Details underlying these conclusions are presented in Sub-Section IId.

(3) Deflection cavity chain: The deflection system of the modified tube has -as before - six cavities. The first five are as in the old design except for modified detuning for each. The last cavity (\#6) - which is also known commonly as the penultimate cavity - is modified dramatically. The issues that impact on e-beam dynamics, and of having a new spectrum of HOMs have been addressed and found to have satisfactory solutions. The new design is presented in Sub-Section IIc.

(4) Two chamber output cavity is the most crucial new component, and was the subject of major efforts during the Phase I design exercise. The design choice should provide (a) acceptable magnitude of the electrical fields inside the cavity, and thus low breakdown probability; (b) low sensitivity to the guiding static solenoidal field, as well as acceptable sensitivity to (c) changes in the loaded $Q$-factor; (d) changes in the sizes of electron beam and (e) in the gun voltage. This is provided by having a two-chamber design whose loaded $Q$-factor $(\sim 200)$ is low, with its first chamber having a length $\sim 2 \mathrm{~cm}$ and operating in the TE311 mode. Solutions have been found to have (1) beam dynamics that delivers an efficient transfer of electron-beam power to RF; (2) an adequate extraction of the produced RF power from the cavity (via waveguides leading from the second chamber), and (3) detuning of high and low order modes so as not to interfere with the power production at or near $34.272 \mathrm{GHz}$. Further details are given in sub-section IIb. 


\section{Omega-P, Inc.}

Final Report on I DoE SBIR grant DE-SC000 4497

\section{MODIFIED MAGNICON FOR HIGH-GRADIENT ACCELERATOR R\&D}

(5) Collector: The tube is to have its collector modified. The new collector will have no ceramic elements - contrary to the present version - and thus, can be subjected to high temperature bakeout. This also eliminates pockets within which spurious collector oscillations might originate. Further details are provided in sub-section IIe.

(6) Systems already in place that will not need to be changed include the low-level stabilized RF source and kW-level TWT driver amplifier (at $11.4 \mathrm{GHz}$ ), (7) diagnostics, and (8) supporting subsystems such as the electron gun, cooling, etc.

Fig. 2 shows a segment of the full tube schematic with the deflection system, output cavity, and the part of the collector adjacent to the rf-cavity chain depicted. The design parameters of the new tube are presented in Table I.

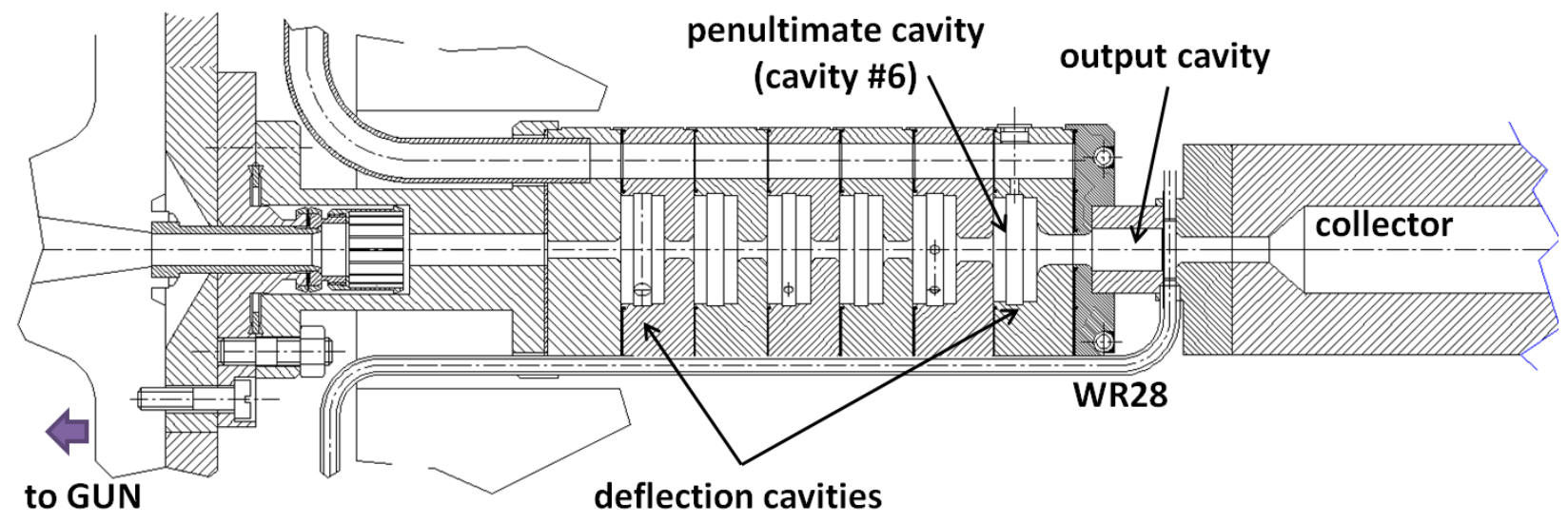

Fig. 2. Modified magnicon: a portion of the full schematic with the deflection system, output cavity and part of the collector adjacent to the rf-cavity chain. There are four waveguides (WR28) connected to the output cavity to extract the power. The installation will be - as is the present one - vertical, with the gun mounted on the top of the oil-filled tank that houses the pulse transformer and gun diagnostics.

Performance: The result of design optimizations, which were done mostly for the new output cavity, for a new last deflection cavity, and (to some extent) for the profile of the guiding magnetic field; resulted in a tube which is robust, and is expected to reliably produce power over a wide range of operating parameters. This would be in marked contrast to the existing Ka-band magnicon which has, since its commissioning, been extremely sensitive to operating parameters. Fig. 3 shows plots for output power and peak surface electric field as functions of loaded $Q$ factor for the new output cavity, for three values of drive power. These results indicate that the level of power going to external loads is highly insensitive to the loaded $Q$-factor. They further indicate that a tolerable surface field $<700 \mathrm{kV} / \mathrm{cm}$ can be obtained if the loaded $Q$-factor does not exceed 250. It should be noted that the tube is designed to work with a $Q$-factor of 200 . 
Omega-P, Inc.

Final Report on I DoE SBIR grant DE-SC000 4497

MODIFIED MAGNICON FOR HIGH-GRADIENT ACCELERATOR R\&D

Table I. Design parameters of the modified magnicon.

\begin{tabular}{|c|c|}
\hline operating frequency (MHz)* & 34,272 \\
\hline output power (MW)* & $20-27$ \\
\hline pulse duration, min-max range, $\mu$ sec & $0.25-1.3$ \\
\hline repetition rate, $\mathrm{Hz}$ & 10 \\
\hline electronic efficiency (\%) & $24-33$ \\
\hline gain (dB) & $52.3-51.3$ \\
\hline drive frequency (MHz)** & $11,427.6$ \\
\hline drive power (Watts) ${ }^{* *}$ & $115-200$ \\
\hline beam power (MW) & 84.15 \\
\hline beam energy (keV), [gun voltage (kV)] & 450 \\
\hline beam current (Amps) & 187 \\
\hline beam diameter (mm) after compression (mm) & 0.9 \\
\hline guiding magnetic field (kG) in deflection system & $11.870-10.280$ \\
\hline guiding magnetic field (kG) in output cavity & $10.280-10.500$ \\
\hline
\end{tabular}

*Extracted from the e-beam via interaction with the $\mathrm{H}_{311} / \mathrm{TM}_{311}$ mode in the output cavity, and directed toward 4 loads via 4 output arms (WR28 waveguides).

**Drive signal is delivered to the $1^{\text {st }}$ deflection cavity; this cavity operates in the $\mathrm{E}_{110} / \mathrm{TM}_{110}$ mode (as is every other deflection cavity). Note, the $1^{\text {st }}$ cavity itself is tuned to $11449.1 \mathrm{MHz}$ [see Sub-Section IIc].
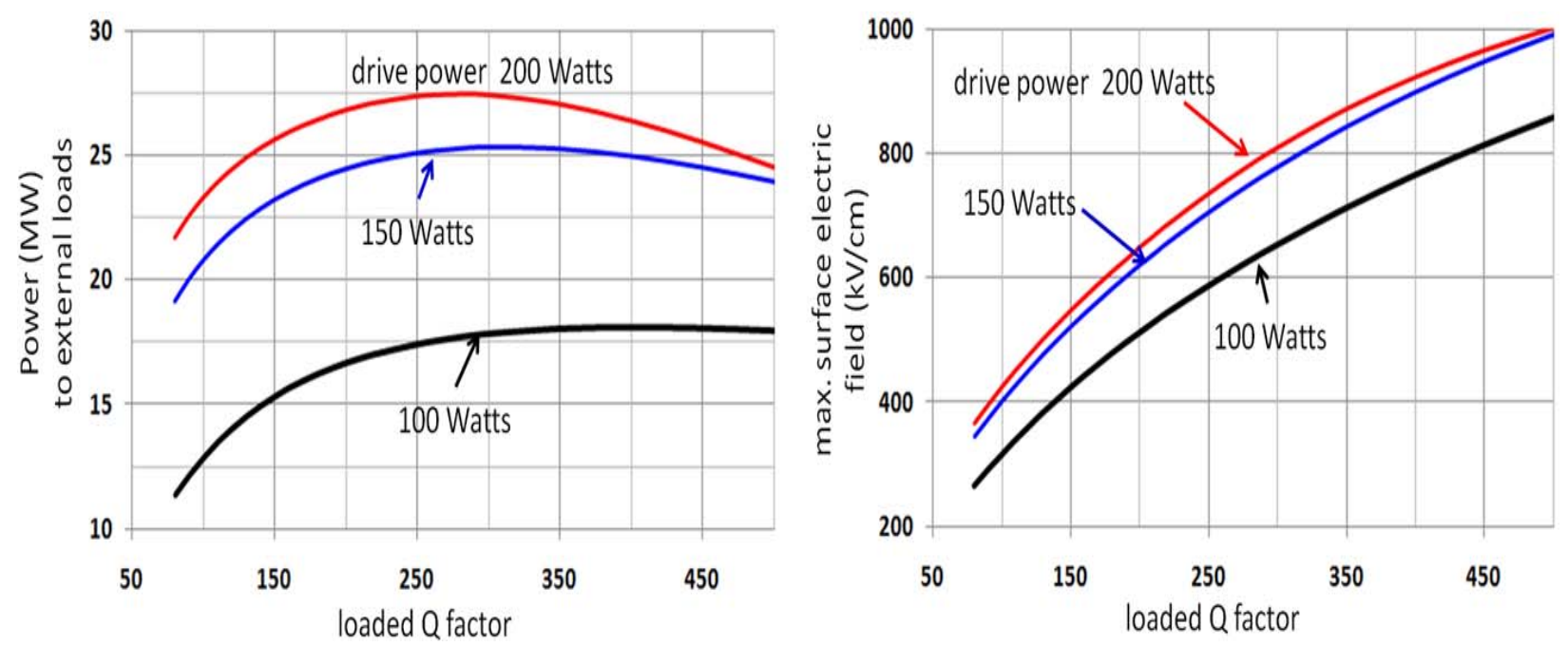

Fig. 3. Power going to external loads is seen to be insensitive to the loaded $Q$-factor of the output cavity (left), and the maximum surface field in the output cavity is still tolerable for a loaded -factor $<250$ (right). For the actual output cavity the loaded $Q$ is to be 200 . 


\section{Omega-P, Inc.}

Final Report on I DoE SBIR grant DE-SC000 4497

\section{MODIFIED MAGNICON FOR HIGH-GRADIENT ACCELERATOR R\&D}

Fig. 4a presents the dependence of the generated power as a function of the frequency of the drive signal. The drive bandwidth is fairly large: $\sim 10-12 \mathrm{MHz}$. Fig. $4 \mathrm{~b}$ shows the so-called drive curve, which is the dependence of generated power $v s$ drive power. It demonstrates that the device is capable to stably generate output power whether the drive signal is small or large. For a reader unfamiliar with the typical behavior of the magnicon in general, it can be useful to note that the produced power grows when the drive power is increased because the larger drive powers give larger deflection angles at the exit of the deflection system [exit of the cavity \#6; see e.g. Fig. 2]; this growth continues to a certain level of the drive power. Then the deflection system saturates (here it happens at $\sim 200 \mathrm{~W}$ ), and the deflection angle at its exit does not get larger even if the drive power is increased; thus the output power stops growing. This is valid up to a certain threshold level of power, after which the deflection system falls in a nonlinear regime and the performances typically deteriorate severely.

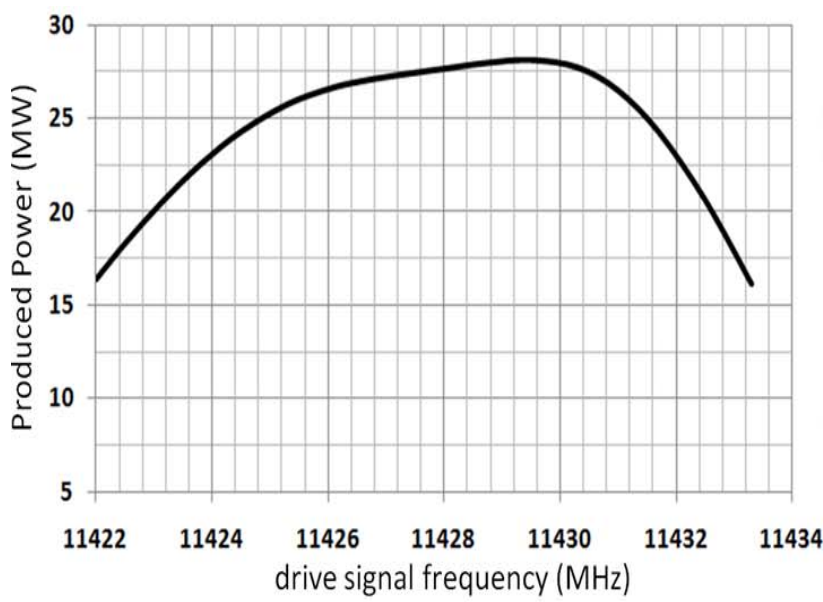

[a]

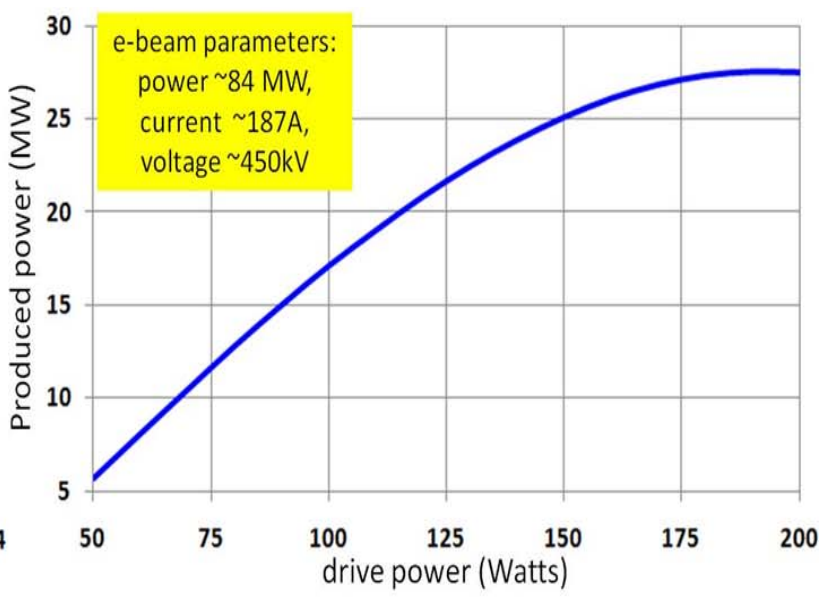

[b]

Fig. 4. (a) Power produced by magnicon $v$ s frequency of the drive signal at a $200 \mathrm{~W}$ drive level; (b) power output $v s$ drive power (the so-called drive curve).

Fig. 5 shows that the magnicon should generate power even if ;he beam is relatively large in diameter; the nominal value is $0.9 \mathrm{~mm}$, which gives an output power of $\sim 27.5 \mathrm{MW}$; yet, with the beam being almost twice larger ( $\sim 2 \mathrm{~mm}$ in diameter) the power is still $\sim 20 \mathrm{MW}$. The righthand side of the same figure demonstrates that changes in the gun voltage $U$ are also tolerated even if, when $U$ changes, the magnetic field is not adjusted to maintain synchronism; here it is taken into account that the beam current is proportional to $U^{3 / 2}$. The nominal value is selected to be $450 \mathrm{kV}$; the power is still above $20 \mathrm{MW}$, when the voltage changes in the range $-10 \mathrm{kV}$ to +17 $\mathrm{kV}$.

The modified magnicon is also highly tolerant to an uncertainty in the positioning of the output cavity [see pictures in sub-section IIb]. Also the new tube is tolerant to quite large changes in the guiding magnetic field [see sub-section IId and Fig. 6 in this sub-section]. 

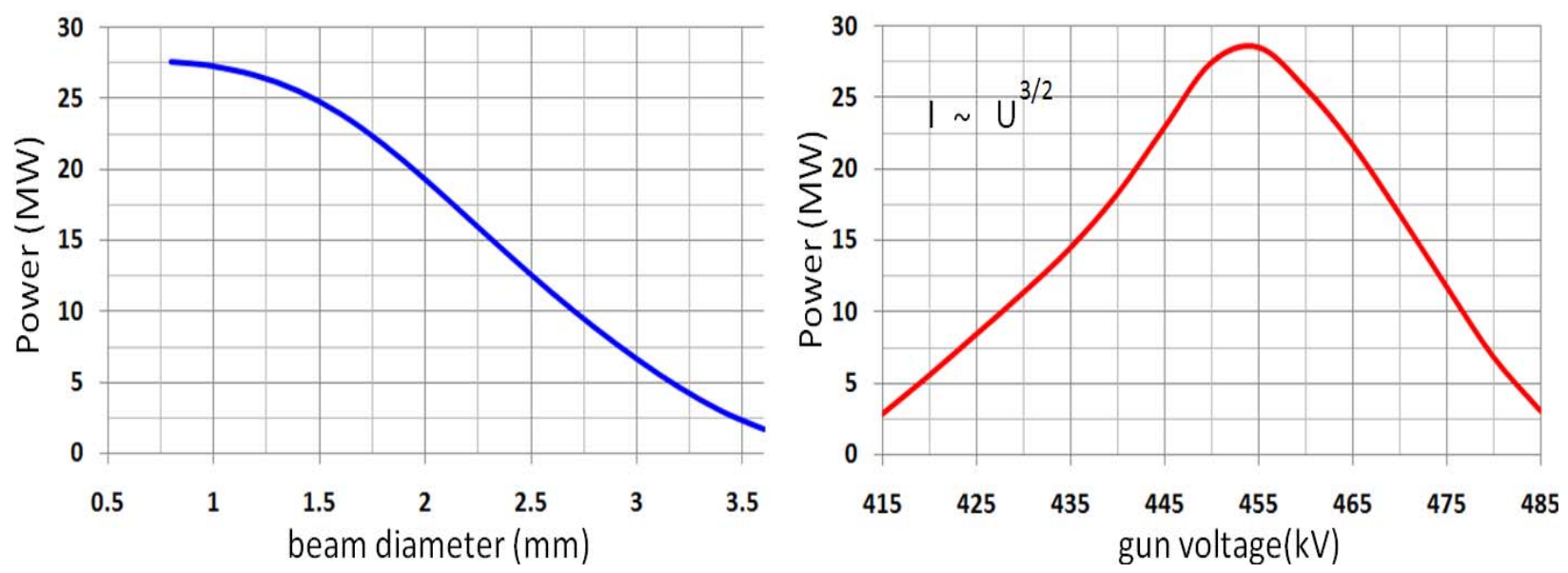

Fig. 5. Dependence of the power (MW) extracted from the electron beam $v s$ the beam diameter $(\mathrm{mm})$ at left, or $v s$ the gun voltage $(\mathrm{kV})$ at right; while the guiding magnetic field is not adjusted

to maintain synchronism. Note that even under such circumstances, remarkably fairly large changes in the gun voltage are tolerated, which demonstrates robustness of the new tube design.

These features are essential to have, especially during conditioning.

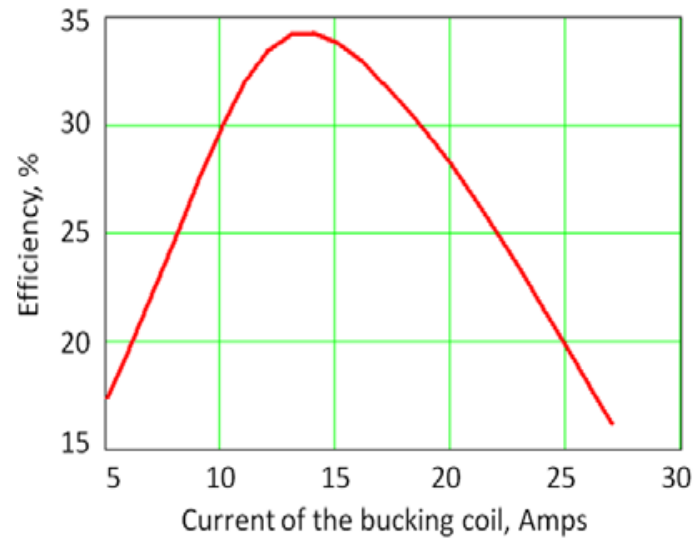

a)

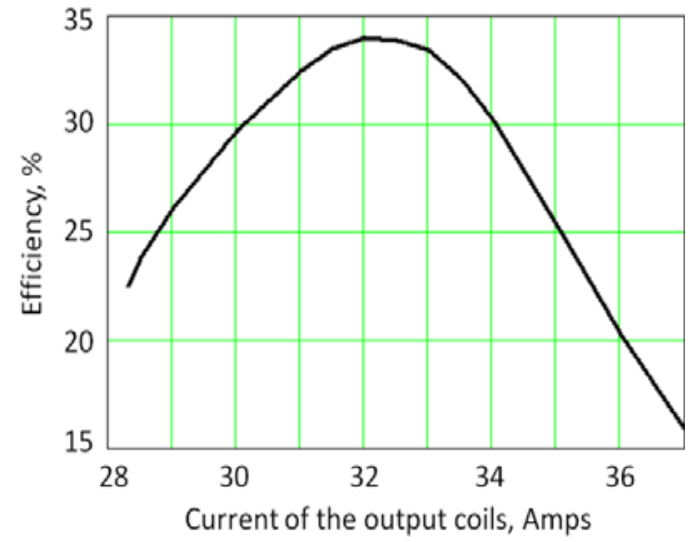

b)

Fig. 6. (a) Efficiency $v s$ the current of the bucking coil, and (b) efficiency $v s$ the current of the output coils wired together in series (totally there are 3 coils). These coils serve to create the guiding magnetic field in the output cavity volume. One observes that large changes in current and thus, magnetic field - can be tolerated.

The overall conclusion one can draw from the summary of results of this study shown here is that the modified magnicon should allow reliable 24/7 operation without extreme sensitivity to operating settings, thus approaching the ideal source of high-power mm-wave RF power as needed by the Test Facility. Details that underlie the results are given in the following sub-sections. 


\section{IIb. New Output Cavity}

The new output cavity is to operate in the $\mathrm{H}_{311} / \mathrm{TM}_{311}$ mode; it has two chambers - the first where the effective interaction with the e-beam occurs, and the second one coupled with the output waveguides (WR28) to extract the generated power. It is possible in this configuration to safely achieve the desired loaded $Q$-factor of 200; but not, however, lower because the presence of the waveguide ports would then bring undesired distortions to the fields, which (even compensated as will be shown further) can be tolerated if $Q$ is not lower than 200. The input drift pipe radius is matched to the radius of the exit drift pipe of the last deflection cavity (cavity \#6) and is $4.25 \mathrm{~mm}$.

The logical process which led to the cavity design as described below involved (in order): (1) choosing the $Q$-factor; (2) selecting a coupling scheme to obtain the desired $Q$-factor; (3) selecting the cavity's longitudinal shape to maximize efficiency; (4) choosing the length of the cavity, and then (5) further optimization to remove unwanted field distortions brought by the coupling ports; (6) providing a clean spectrum of modes near 34,272 GHz not to have any modes which may interfere when the cavity is tuned and/or operated; (7) de-tuning high and low order modes (all the above while preserving the $Q$-factor and the operating frequency). Of course, certain steps were based on the specific prior knowledge. Below the result of the aforementioned procedure is given. In the interest of economy of exposition, the details of the process itself are not presented here, but moved to Sub-Section IIf so as not to overload this sub-section with high level of details.

\section{New output cavity design, parameters, and performance}

The proposed cavity design is shown in Fig. 7(a,b), its parameters are given in Table II, and its dimensions are given in Table III.

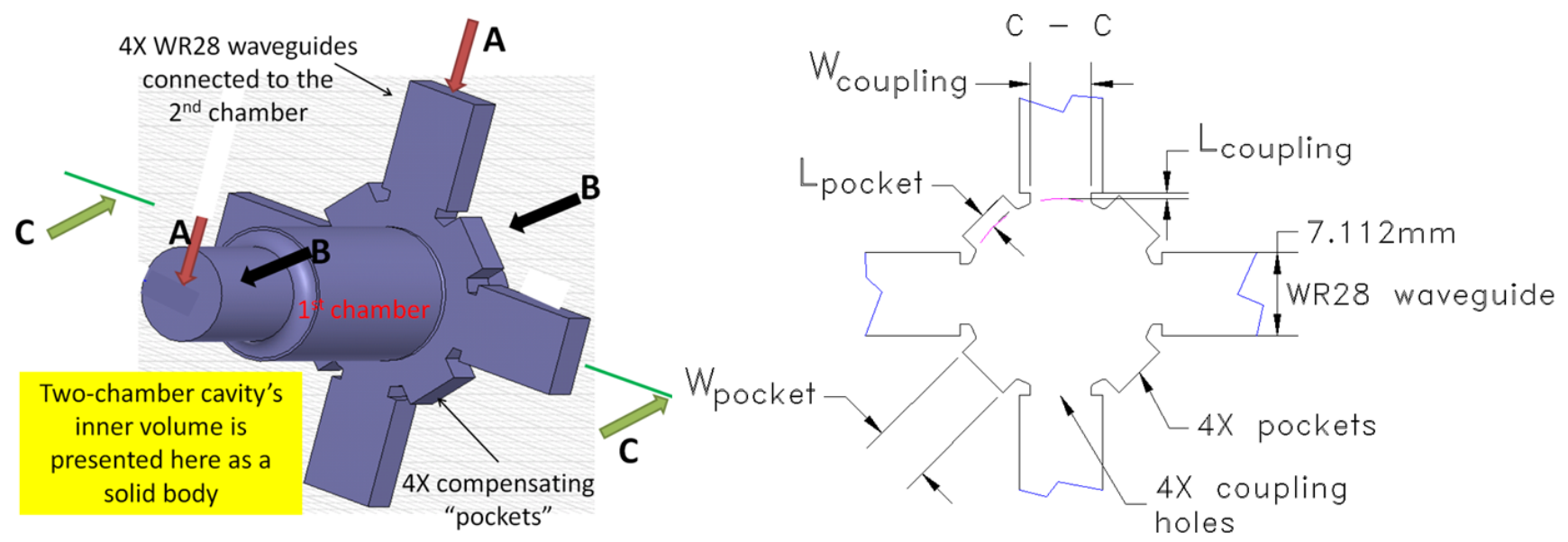

Fig. 7(a). Ouline of the new output cavity (left), and cross section through plane C-C. 
Omega-P, Inc.

Final Report on I DoE SBIR grant DE-SC000 4497

MODIFIED MAGNICON FOR HIGH-GRADIENT ACCELERATOR R\&D

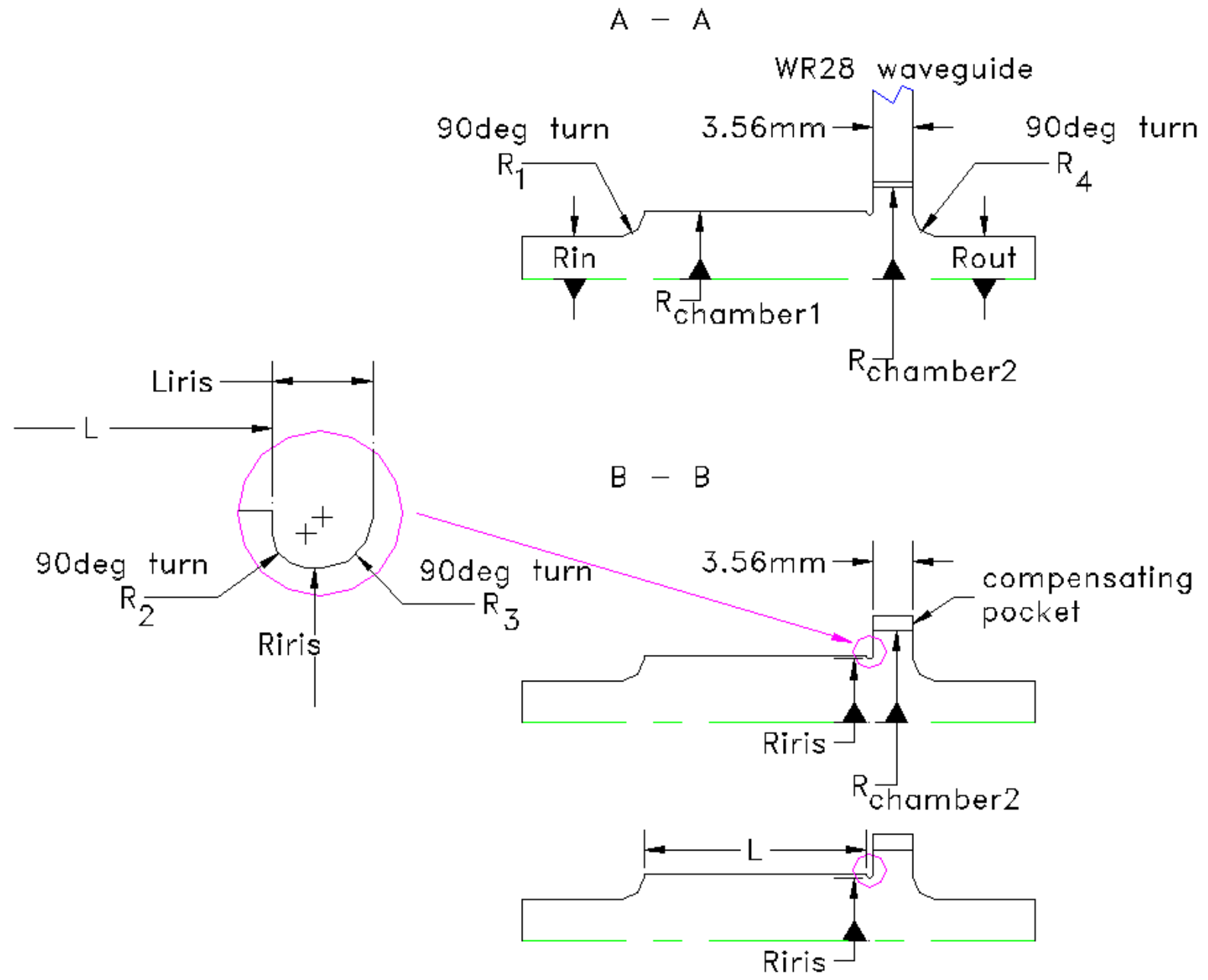

Fig. 7(b): Design of output cavity [see Tables 2.1 and 2.2].

Table II. Projected parameters of output cavity

\begin{tabular}{|ll|l|}
\hline operating mode $\ldots \ldots \ldots \ldots$. & $\mathrm{H}_{311} / \mathrm{TE}_{311}$ & efficiency $(\%) \ldots \ldots \ldots \ldots \ldots \ldots \ldots .24-33$ \\
\hline frequency $(\mathrm{MHz}) \ldots \ldots \ldots \ldots$ & 34,272 & power $(\mathrm{MW}) \ldots \ldots \ldots \ldots \ldots \ldots \ldots .20-27.5$ \\
\hline loaded $Q$-factor $\ldots \ldots \ldots \ldots$. & 200 & max. electric field $(\mathrm{kV} / \mathrm{cm}) \ldots \ldots . \quad 550-650$ \\
\hline
\end{tabular}

* Typical intrinsic $Q$-factor attributed to the finite conductivity of the copper walls is $\sim 8,100$. 
Omega-P, Inc.

Final Report on I DoE SBIR grant DE-SC000 4497

MODIFIED MAGNICON FOR HIGH-GRADIENT ACCELERATOR R\&D

Table III. Sizes of output cavity features (in mm) [see Fig. 7].

\begin{tabular}{|cl|ll|}
\hline $\mathrm{L}$ & 21.12 & $\mathrm{R}_{1}$ & 1.5 \\
\hline R chamber1 & 6.027 & $\mathrm{R}_{2}$ & 0.2 \\
\hline R chamber2 & 8.130 & $\mathrm{R}_{3}$ & 0.3 \\
\hline L iris & 0.6 & $\mathrm{R}_{4}$ & 1.5 \\
\hline $\mathrm{R}$ iris & 5.7 & L coupling & 0.5 \\
\hline $\mathrm{R}$ in & 4.25 & W coupling & 5.2 \\
\hline R out & 3.75 & L pocket & 1.5 \\
\hline & & W pocket & 5.2 \\
\hline
\end{tabular}

The required diameter-wise tolerances on all the features must be $5 \mu \mathrm{m}$ or better; other features must have their sizes machined with an accuracy $10 \mu \mathrm{m}$ or better.

There is a neck formed by the iris separating the first and second chambers; even though this feature is relatively shallow, it is essential to form the field profiles in the $1^{\text {st }}$ chamber that resembles those of a simple pillbox cavity, which is essential because these have been found to be the best to deliver an efficient interaction. The compensating pockets are even of a greater importance to have fields nearly optimal for efficient interaction with the e-beam. The dimensions of compensating pockets can be chosen so that the fields in the $1^{\text {st }}$ chamber closely resemble that of a pure $\mathrm{TE}_{311} / \mathrm{H}_{311}$-mode [see Fig. 8, the field images in cut-planes 2 and 3] even though in the $2^{\text {nd }}$ chamber the fields are distorted [see the field image in cut-plane 4]. These distortions are the result that four waveguides must be used (for compatibility with the present facility); the rotating $\mathrm{TE}_{311} / \mathrm{H}_{311}$ operating mode is formed by two orthogonal linear polarizations, and each connects to a pair of waveguides disposed $180^{\circ}$ apart, which thus superimposes a dipole-like azimuthal perturbations on the sextuple-type mode. With $Q \sim 200$, the compensating pockets can be used to reduce distortions greatly; yet, dipole-character residual field-components remain, as can be seen by inspecting the field patterns in the drift pipes [see e.g. the field image in cut plane 1]. Effects of the presence of this dipole residue are described below. Having the loaded $Q$-factor below 200 results in too great a dipole-character in the residual field-components. 

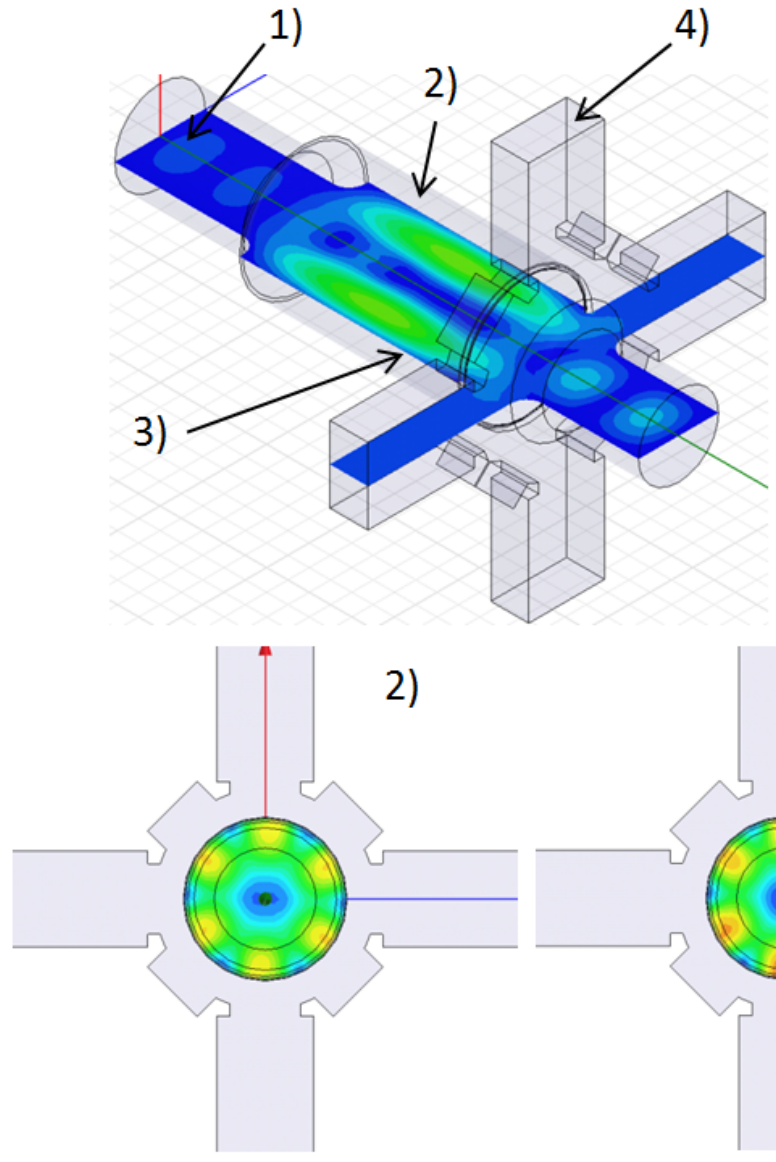

3)

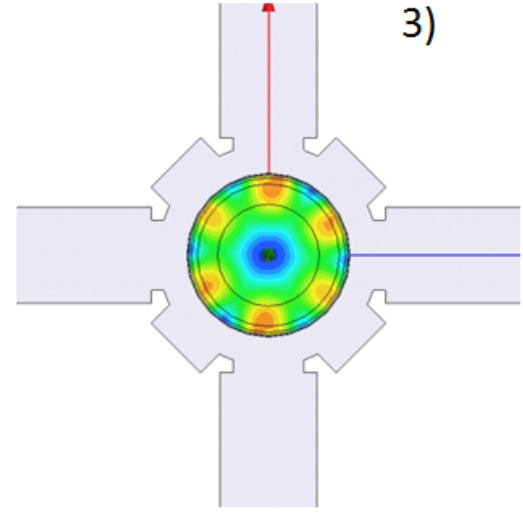

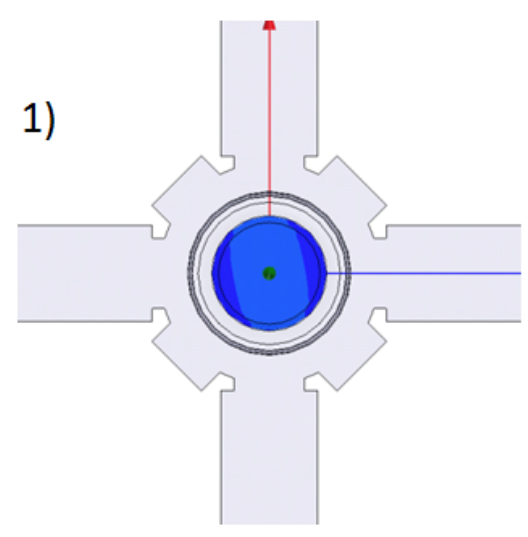

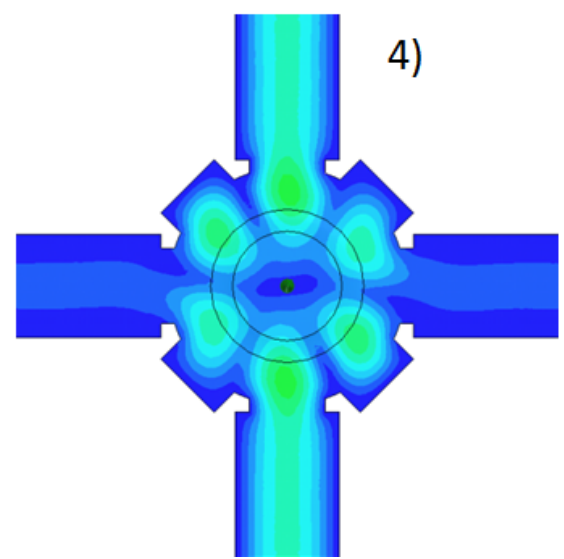

Fig. 8. The complex magnitude of total electric field is shown. In the $1^{\text {st }}$ chamber where the interaction between the e-beam and RF-field is to occur, the fields (see e.g. cut-planes 2 and 3) closely resemble that of a simple pillbox cavity even thought there are dipole-character fieldcomponents present, as can be seen by inspecting the field patterns in e.g. the entrance drift pipe (cut-plane 1). (Simulations are done with HFSS v.12).

The profiles $E \varphi v s z$ are shown at different radii in Fig. 9. It is seen that in the sextupolecharacter fields, dipole-like components are present; they propagate down/up both drift pipes, but small. This dipole residue influences somewhat the fields inside the cavity, in particular at $r=2$ $\mathrm{mm}$ where the e-beam resides mostly when interacting with RF. However, it appears that $E \varphi$ electric-field profiles - being the ones important to have an interaction between the electron beam and RF - always closely resemble those of a simple pillbox cavity [see Fig. 10] whose sizes are listed in Table IV. 
Omega-P, Inc.

Final Report on I DoE SBIR grant DE-SC000 4497

MODIFIED MAGNICON FOR HIGH-GRADIENT ACCELERATOR R\&D

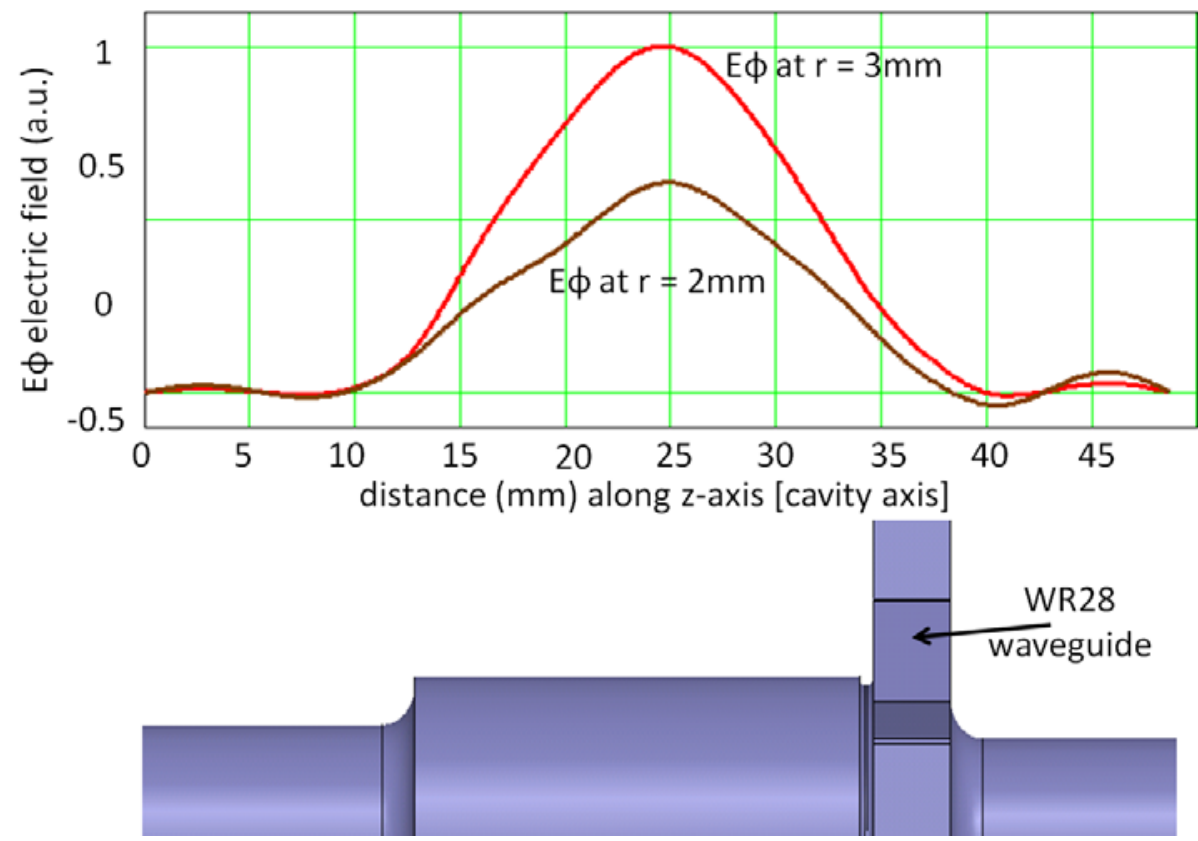

Fig. 9

Fig. 9. The profiles $E \varphi v s z$ at different radii in the output cavity with parameters as per Tables 2.1 and 2.2. The e-beam typically dwells around $r=2 \mathrm{~mm}$ (brown curve), but enters the output cavity at $r \sim 3 \mathrm{~mm}$. The dipole-like field components present, and propagate down/up both drift pipes. This dipole residue influences somewhat the fields inside the cavity, but are tolerable.

(See explanations in the text.)

Table IV. Sizes (mm) of the simple pillbox equivalent [to be used with Fig. 10]

\begin{tabular}{|cc|cc|}
\hline $\mathrm{L}^{*}$ & 23 & $\mathrm{R} 1^{*}$ & 2 \\
\hline $\mathrm{R}^{*}$ & 6.0 & $\mathrm{R} 2^{*}$ & 2 \\
\hline $\mathrm{R}$ in* & 3.75 & Rout $^{*}$ & 3.75 \\
\hline
\end{tabular}



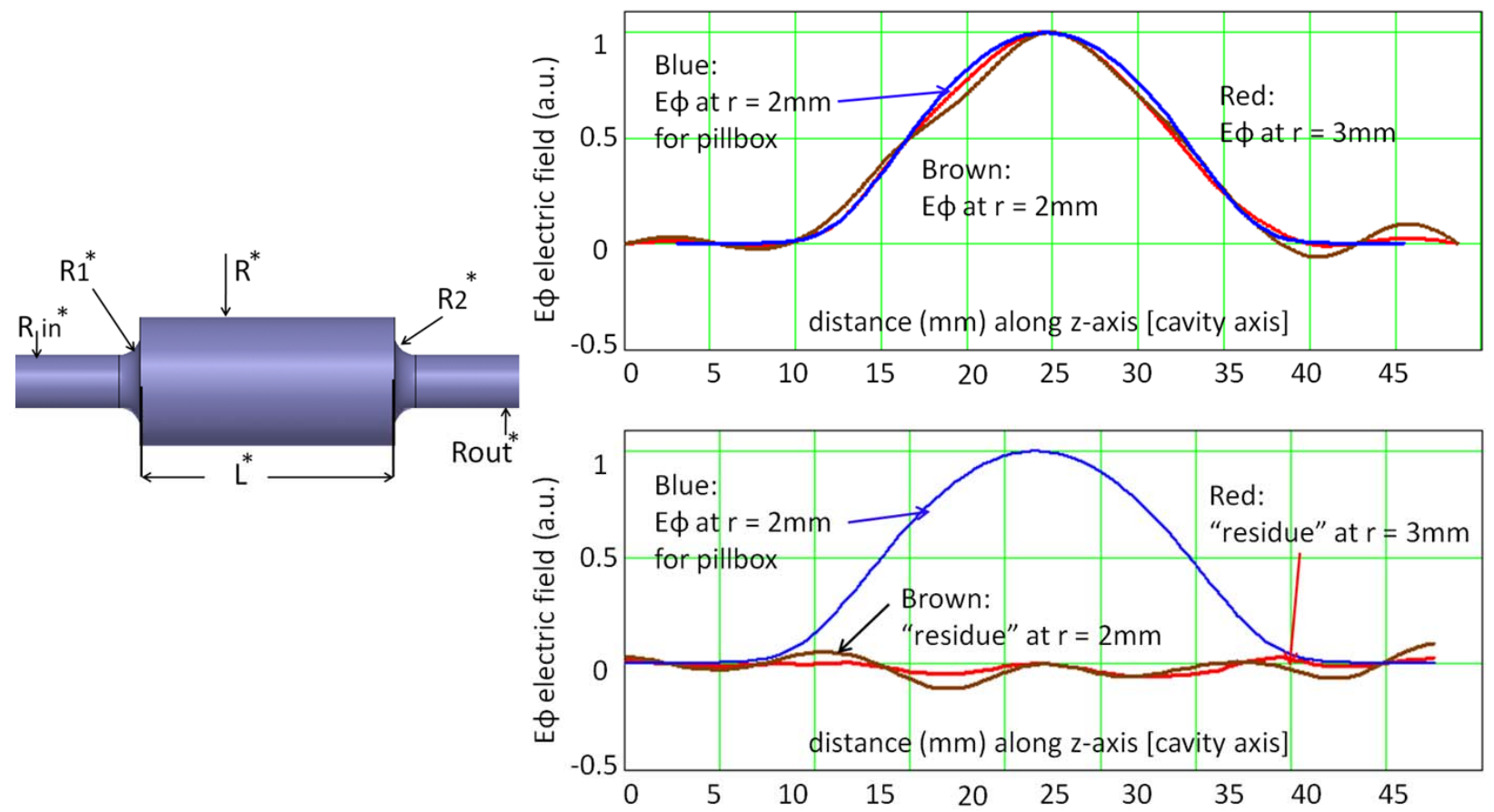

Fig. 10. The profiles $E \varphi v s z$ shown before in Fig.2.3 appear almost the same as the $E \varphi$ profile of a simple pillbox cavity with length $=23 \mathrm{~mm}$ (shown at left); blue curve gives the profile for the pillbox cavity [simulations by CLANS2, 2003 release]; the red and brown curves on the top, right-hand side plot are the profiles for the two-chamber cavity at $r=2$ and $3 \mathrm{~mm}$ normalized to unity. The bottom right-hand-side plot presents the residual fields taken as a difference: (bottom red curve $)=($ top red curve $)-($ blue curve $)$, and (bottom brown curve $)=($ top brown curve $)-$

(blue curve).

Using the pillbox equivalent, the performances can be predicted [see Fig. 11]. Note that having an efficiency of $24 \%$ leads to a maximum surface electric field of $550 \mathrm{kV} / \mathrm{cm}$; pushing to $33 \%$ leads to $650 \mathrm{kV} / \mathrm{cm}$.

To demonstrate the robustness of the modified tube equipped with the new output cavity, it is illustrative to show how the generated power changes when one changes the position of the output cavity relative to the rest of the device. This is presented by Fig. 12 .

Lastly, we note that a weak dependence of the $\mathrm{H}_{311} / \mathrm{TE}_{311}$ frequency on the length of the $1^{\text {st }}$ chamber (only about $-40 \mathrm{MHz} / \mathrm{mm}$ ) presents a good opportunity to tune the cavity just by adjusting the $1^{\text {st }}$ chamber's length - a very straightforward operation to accomplish before portions of the cavity are brazed. 
Omega-P, Inc.

Final Report on I DoE SBIR grant DE-SC000 4497

MODIFIED MAGNICON FOR HIGH-GRADIENT ACCELERATOR R\&D
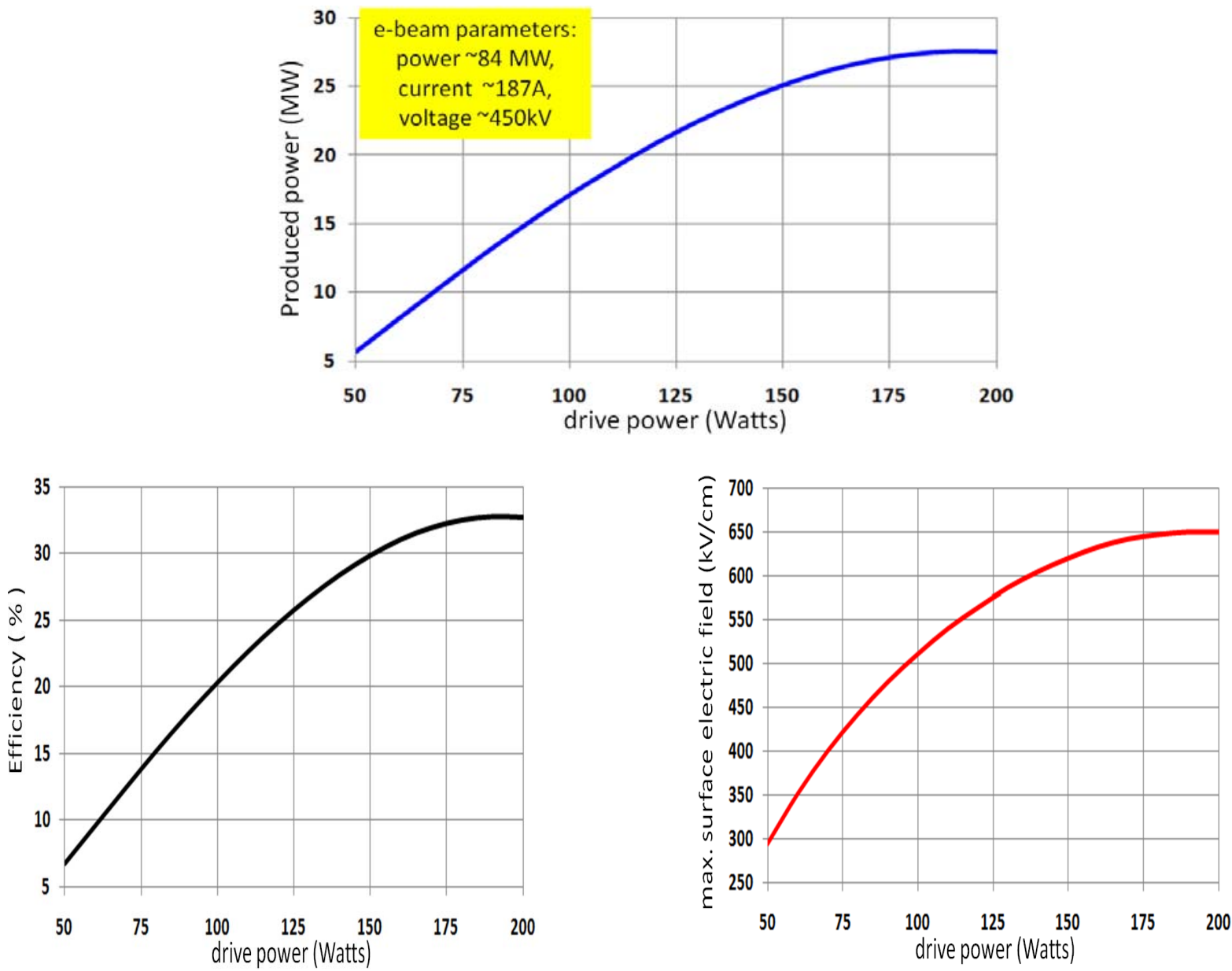

Fig. 11. Performances of the two-chamber output cavity computed by using its equivalent model compatible with the MGC v.4.15 program. Thanks to the low $Q$-factor of the output cavity $(\sim 200)$, most of the produced power goes to external loads.

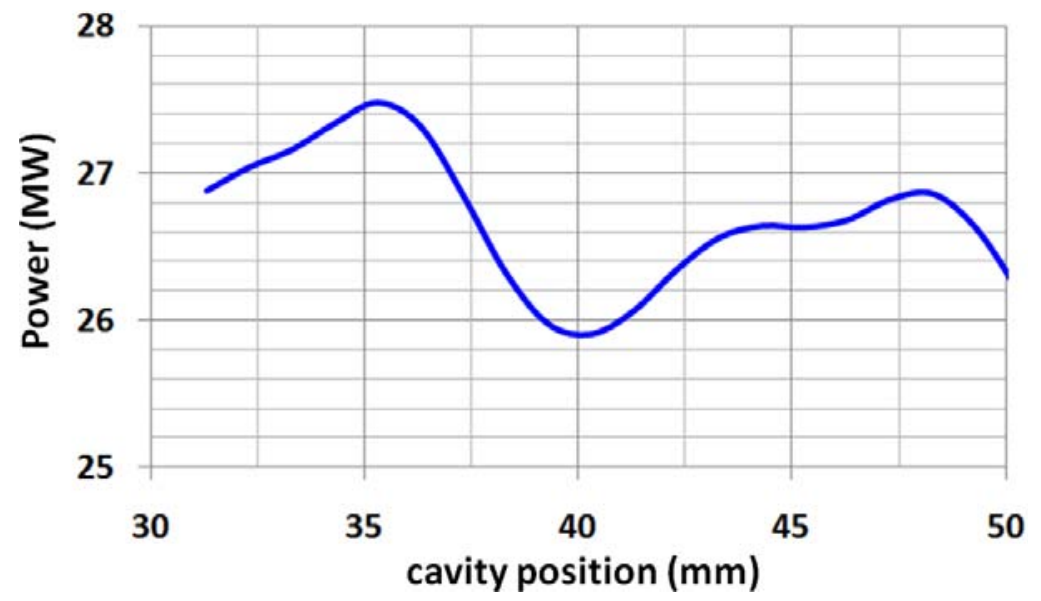

Fig. 12. Power generated in the output cavity $v s$ the position of output cavity (as appears in the "Position Z min" input line in MGC v4.15, converted here to $\mathrm{mm}$ ). The set-position where cavity nominally rests is $35.3 \mathrm{~mm}$. High power is generated even if the position changes by many $\mathrm{mm}$. 
Omega-P, Inc.

Final Report on I DoE SBIR grant DE-SC000 4497

MODIFIED MAGNICON FOR HIGH-GRADIENT ACCELERATOR R\&D

\section{Spectrum of the cavity in the vicinity of the operational frequency}

The mode spectrum of the output cavity was computed using HFSS v.12. In this subsection, all the modes specified below are trapped inside the output cavity volume. The other modes (those which are not trapped or high/low order modes are described in subsequent sections). $\mathrm{E}_{013} / \mathrm{TM}_{013}$ was found to be in the close proximity to the working mode (after studying the spectrum in the range from $33.5 \mathrm{GHz}$ to $\sim 35 \mathrm{GHz}$ ) and may present a problem when the cavity is tuned. It is not coupled with waveguides, however, and thus should not be seen on the transmission/reflection/S11/S21 curves when one uses the waveguides to check the spectrum. If a mock-up of the cavity is used and excited by loops to see the operational $\mathrm{TE}_{311} / \mathrm{H}_{311}$ mode, one may chose the locations of the loop-holes by consulting with e.g. Fig. 13a so that to position the loops at places where they will not couple to this $\mathrm{E}_{013} / \mathrm{TM}_{013}$ mode. However, it may be perhaps still desirable to have the cavity sizes so that this mode is de-tuned from the working frequency. Fig. 13b shows another mode whose fields are strongly perturbed by coupling with waveguides; thus, without specifying its mode type (TE or TM), it is more convenient to merely mark it by $1 \times 3$ to conveniently indicate that it has one azimuthal variation and three variations along the $z$ axis inside the output cavity volume. This is a dipole-type mode; if too close to the operating frequency it may couple with the working mode, which has small dipole-like field components, and cause field perturbations leading to a lower efficiency.

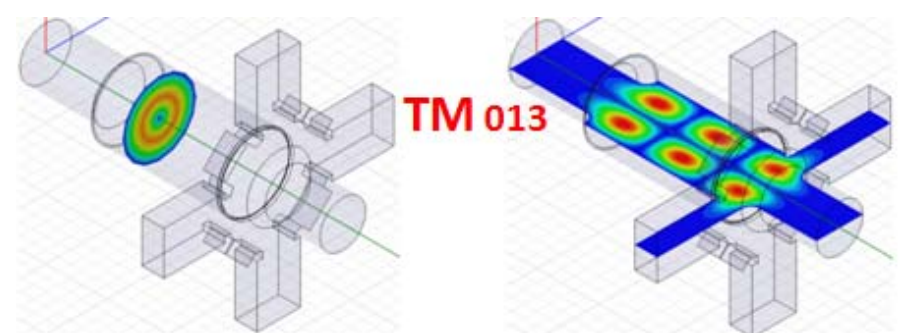

Fig. 13a. $\mathrm{TM}_{013}$ mode (this is a monopole mode); typically it has $Q \sim 15,000$ if the cavity is made out of copper.

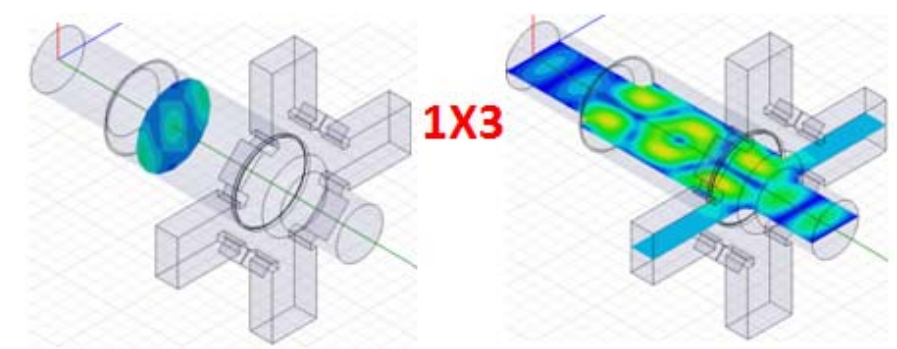

Fig. 13b. " $1 \times 3$ " mode (two orthogonal polarizations present; one is shown); this mode is also somewhat coupled with the drift pipes; $Q$-factors $\sim 300-400$. 


\section{Omega-P, Inc.}

Final Report on I DoE SBIR grant DE-SC000 4497

\section{MODIFIED MAGNICON FOR HIGH-GRADIENT ACCELERATOR R\&D}

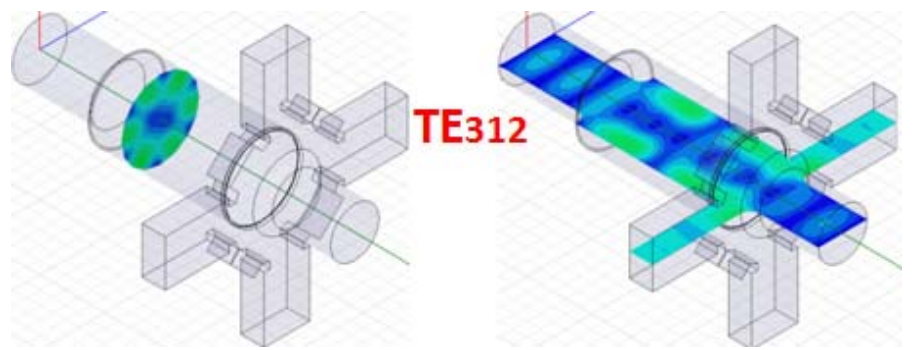

Fig. 13c. TE $\mathrm{TE}_{312}$ mode (two orthogonal polarizations present; one is shown); this is the sextupletype mode that might get excited if is close to $34,272 \mathrm{GHz}$. However, with the cavity dimensions as selected it sits $\sim 1.5 \mathrm{GHz}$ above the operating $34,272 \mathrm{GHz}$. (The $Q$-factor is $\sim 70$.) (Note, in all plots in Fig.2.7, the complex magnitude of total electric field is shown as given by HFSS v.12 simulations.)

$\mathrm{H}_{312} / \mathrm{TE}_{312}$ might get excited if it happens to be close to the operating frequency of $34,272 \mathrm{GHz}$ because the azimuthal number of this mode is three; thus, it must receive close attention. This mode is depicted in Fig. 13c.

Frequency Maps: The frequency maps $v s$ cavity length $L$ (the length of the $1^{\text {st }}$ chamber) and $\mathrm{R}$ chamber1 (the radius of the $1^{\text {st }}$ chamber) are given in Fig. 14 . Note that the mode " $1 \times 3$ " appears with uncertainty of $-0 /+145 \mathrm{MHz}$; this uncertainty was found in HFSS simulations when the boundary conditions at the pipe's ends change from "perfect E-wall" to "perfect H-wall" which corresponds to a line impedance transformation delivered by a $\lambda / 4$ long line. Deviations vs length are $-42,-253,-400$ and $-183 \mathrm{MHz} / \mathrm{mm}$ for the modes $\mathrm{TE}_{311}, \mathrm{TM}_{013}$, “ $1 \times 3$ " and $\mathrm{TE}_{312}$ respectively; deviations vs radius are $-5256,-3040,-3660$ and $-4364 \mathrm{MHz} / \mathrm{mm}$ for the modes $\mathrm{TE}_{311}, \mathrm{TM}_{013}$, "1X3" and $\mathrm{TE}_{312}$ respectively. It can be seen that the modes in question are sufficiently de-tuned; for instance, even $\mathrm{TM}_{013}$ is detuned by $190 \mathrm{MHz}$.

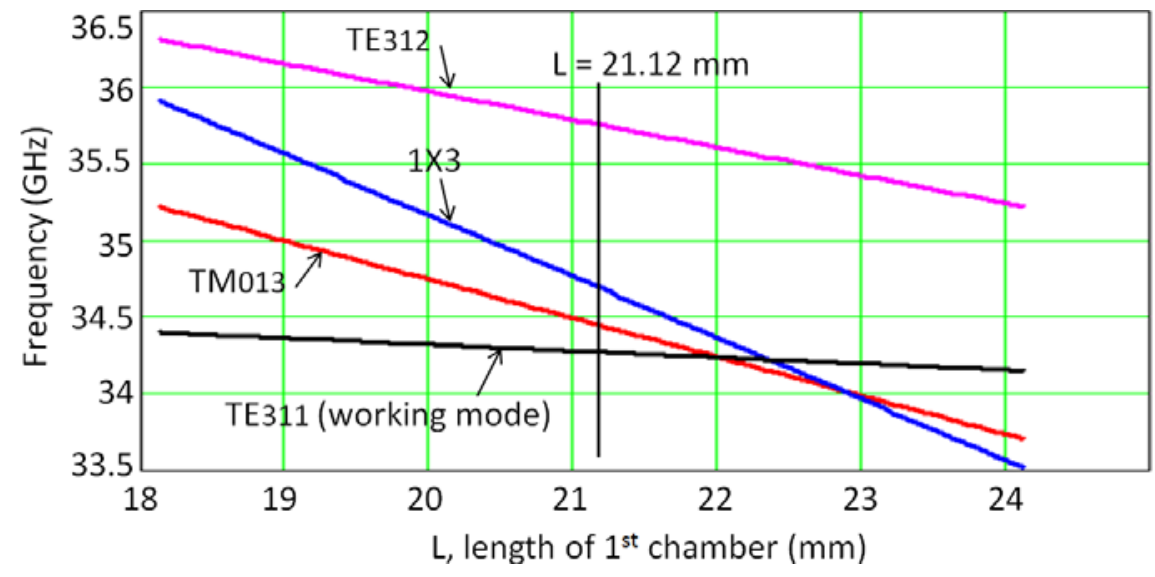


Omega-P, Inc.

Final Report on I DoE SBIR grant DE-SC000 4497

MODIFIED MAGNICON FOR HIGH-GRADIENT ACCELERATOR R\&D

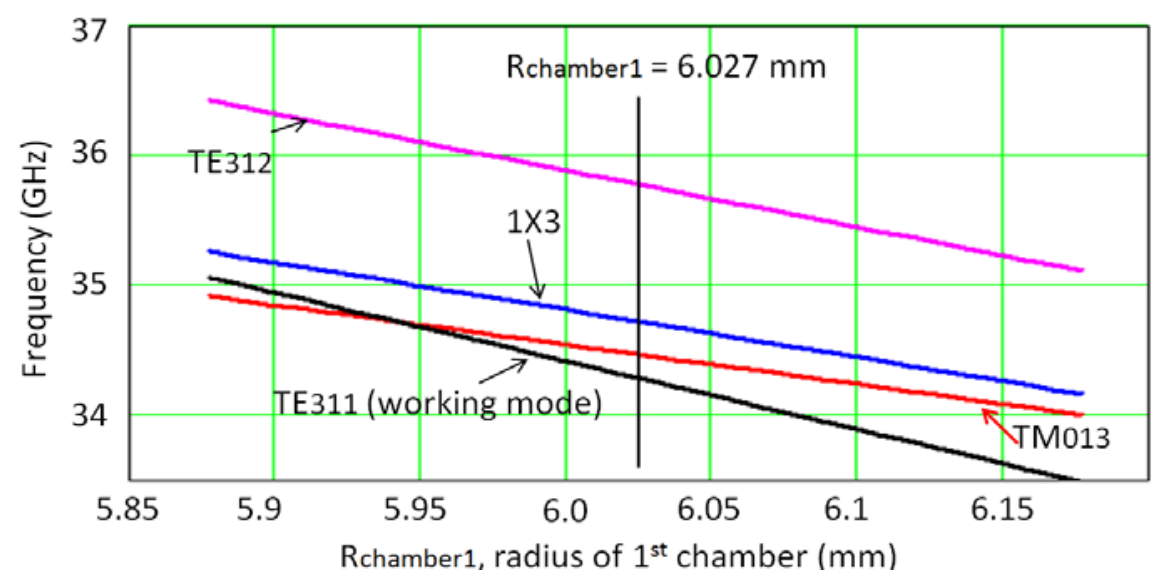

Fig. 14. The spectrum: frequencies of the modes which are depicted in Fig. 13. The working $\mathrm{H}_{311} / \mathrm{TE}_{311}$ mode is shown by the black vertical line. It appears that all modes are sufficiently de-tuned. For instance, $\mathrm{TM}_{013}$ is detuned by $190 \mathrm{MHz}$.

\section{Spectrum of High Order and Low Order modes}

Each high or low order mode (HOM or LOM) which is trapped in the output cavity and has its frequency in the vicinity of the harmonic frequency $f_{\mathrm{m}}=m \times 11,424 \mathrm{MHz}$, where $m$ is the mode azimuthal index, might get excited if the de-tuning between the mode frequency and the harmonic frequency is not large enough. Another essential requirement, however, is that the field strength of such a mode in the region where the electron beam moves must be sufficient for interaction to occur. Because of that, in general, the modes with $m$ above 8 cannot be excited. Should a high/low order mode be excited, the efficiency of power production at $34,272 \mathrm{MHz}$ may drop significantly even if the actual amount of power going from the electron beam to the high/low order mode itself is relatively small. The recipe to fight against these modes is well known. It requires a proven software tool (e.g. HFSS) to perform accurate simulations with subsequent manufacturing of cavity mock-ups, with each mock-up either having its inner features being exact replicas of the actual cavity, or being scaled so that the proportions between the features are preserved. The mock-ups are to be used to compare the theory model predictions with the actual mode spectrum or the mode spectrum adjusted to take into account the scaling coefficient and if discrepancies are found to understand and eliminate them. The result of both simulations and measurements together provide sufficient information to indentify and de-tune the unwanted modes. 


\section{Omega-P, Inc.}

Phase II proposal submitted following Phase I DoE SBIR grant DE-SC000 4497

in response to DoE SBIR/STTR 2010 Solicitation

Topic 65c: RADIO FREQUENCY POWER SOURCES AND COMPONENTS

$R F$ Sources for High-Gradient Accelerator Research

MODIFIED MAGNICON FOR HIGH-GRADIENT ACCELERATOR R\&D

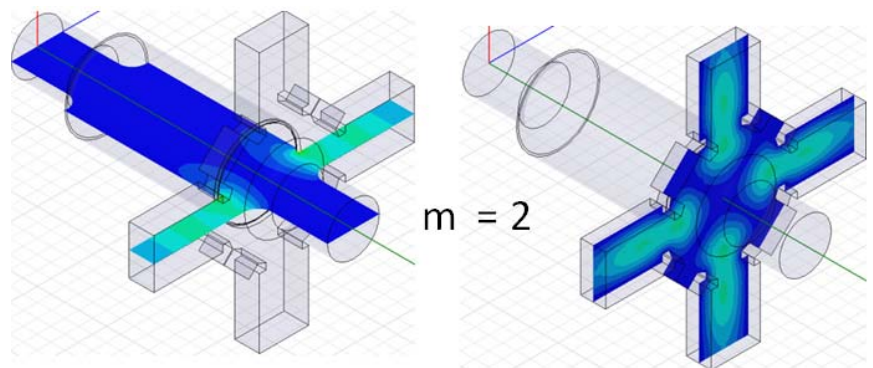

Fig. 15. LOM with $m=2$ which can interact with the e-beam if not de-tuned (see the text for details); however presently, it is de-tuned by at least $600 \mathrm{MHz}$ (simulations by HFSS v.12; complex magnitude of total electric field is plotted).

In this sub-section, we start addressing the first step of the aforementioned investigation, namely to simulate the spectrum, identify the parasitic modes near respective harmonic frequencies, and apply efforts to de-tune the parasitic modes sufficiently. The simulations showed that the nearest dipole mode to $11,242 \mathrm{MHz}$ is detuned by $\sim 4 \mathrm{GHz}$. The nearest quadruple mode to $22,848 \mathrm{MHz}$ is detuned by $\sim 600 \mathrm{MHz}$; the fields of this mode are depicted in Fig. 15. The nearest parasitic mode (with $m=3$ ) to $34,272 \mathrm{MHz}$ has been already presented in sub-section above; it is $\mathrm{H}_{312} / \mathrm{TE}_{312}$ mode and is de-tuned by $1.5 \mathrm{GHz}$. None of these should be excited. A mode with $m=4$, which exists only in a single linear polarization, was found in the vicinity of $45,696 \mathrm{MHz}$ [see Fig. 16]. It is somewhat weakly coupled with the drift tubes, thus having its de-tuning ranging from -190 to $-162 \mathrm{MHz}$; the $Q$-factor is $\sim 1000$. Aside from this mode, no others were found near $45,696 \mathrm{MHz}$ in the range $-396 /+271 \mathrm{MHz}$.

However, the aforementioned mode being linearly polarized may not maintain a synchronous interaction with the e-beam. The actual influence on the power production at 34,272 $\mathrm{MHz}$ is non-essential if the de-tuning exceeds $150 \mathrm{MHz}$ (negative, see Fig. 17). Additional work will require an accurately built mock-up of the cavity (with scale 1:1 for inner features) to identify this mode and prove that its de-tuning exceeds the required limit.

Thus, for the proposed version of the cavity, no HOMs were found in the vicinity of the respective harmonic frequencies within the ranges as shown in Table V; the ranges are selected so that to insure no excitation when outside the range. 


\section{Omega-P, Inc.}

Phase II proposal submitted following Phase I DoE SBIR grant DE-SC000 4497 in response to DoE SBIR/STTR 2010 Solicitation

Topic 65c: RADIO FREQUENCY POWER SOURCES AND COMPONENTS

$R F$ Sources for High-Gradient Accelerator Research

\section{MODIFIED MAGNICON FOR HIGH-GRADIENT ACCELERATOR R\&D}
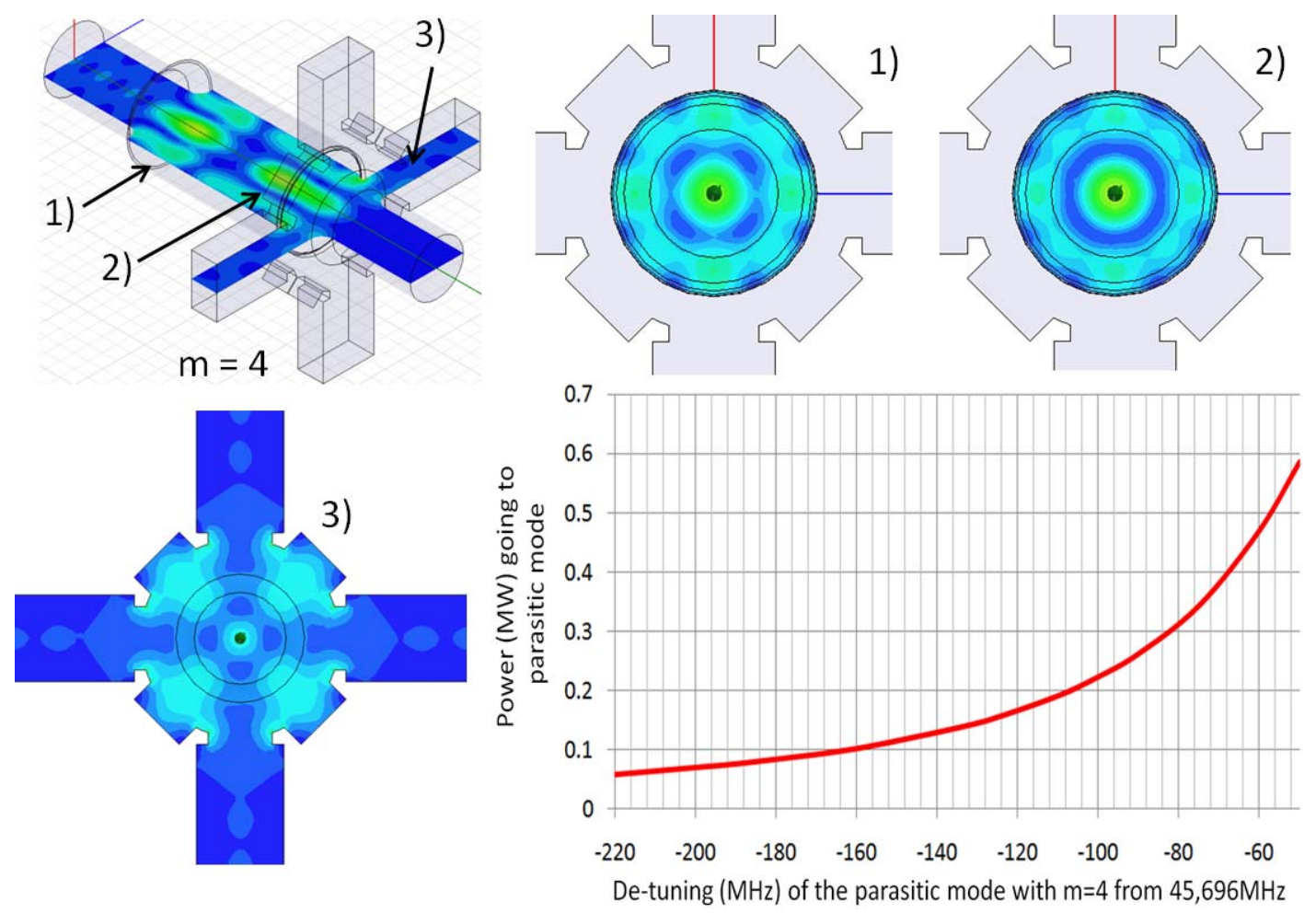

Fig. 16. HOM with $m=4$ which can interact with the e-beam if not de-tuned (see the text for details); presently, it is de-tuned by $\sim 190 \mathrm{MHz}$ (field patterns are computed by HFSS v.12; complex magnitude of total electric field is plotted; dynamics simulations are by MGC v.4.15)

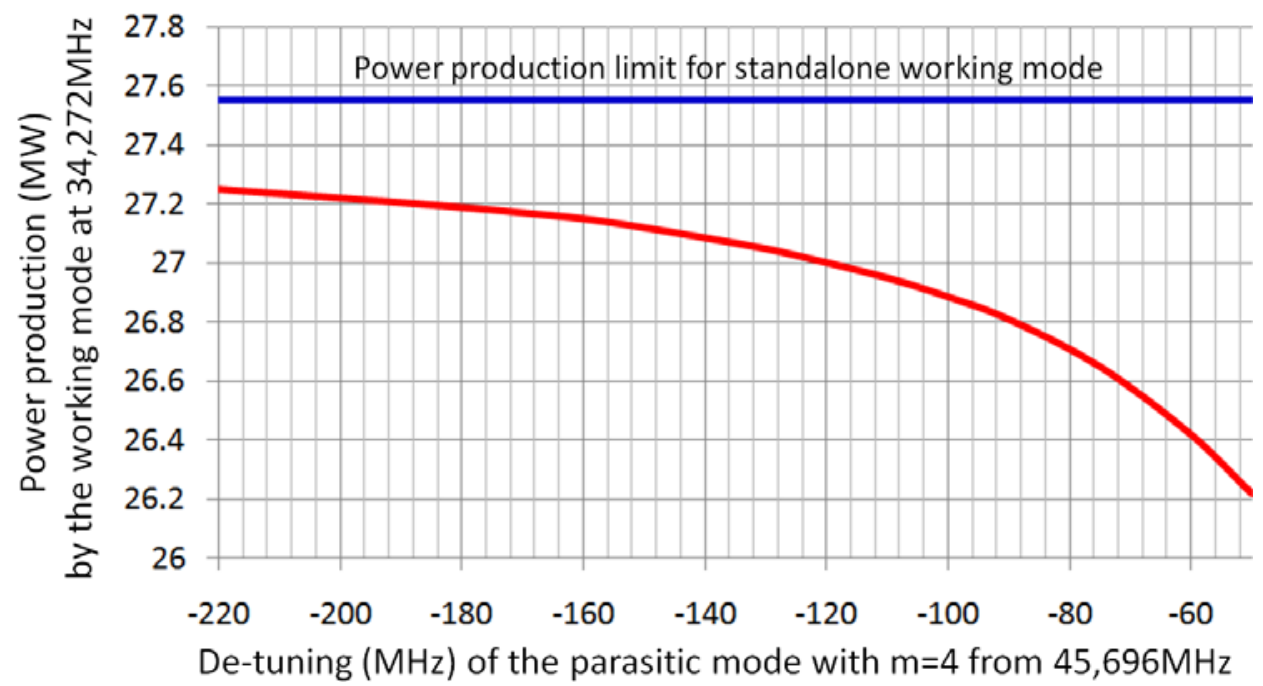

Fig. 17. Influence on power production at $34,272 \mathrm{MHz}$ is non-essential if the de-tuning of the parasitic mode exceeds $150 \mathrm{MHz}$ (negative). 
Omega-P, Inc.

Phase II proposal submitted following Phase I DoE SBIR grant DE-SC000 4497

in response to DoE SBIR/STTR 2010 Solicitation

Topic 65c: RADIO FREQUENCY POWER SOURCES AND COMPONENTS

RF Sources for High-Gradient Accelerator Research

MODIFIED MAGNICON FOR HIGH-GRADIENT ACCELERATOR R\&D

Table V. (no HOMs with specified $m$-number were found within the ranges as shown)

\begin{tabular}{|c|c|c|c|c|}
\hline azimuthal index, $m$ & 5 & 6 & 7 & 8 \\
\hline$f_{\mathrm{m}}$, harmonic frequency $(\mathrm{MHz})$ & 57,120 & 68,544 & 79,968 & 91,392 \\
\hline search range $(\mathrm{MHz})$ & $-300 /$ & $-300 /$ & $-308 /$ & $-332 /$ \\
& +270 & +300 & +325 & +397 \\
\hline
\end{tabular}

Thus, the overall conclusion for this sub-section is that the HOMs/LOMs are de-tuned sufficiently enough not to present associated problems.

\section{Other modes}

There is a variety of possible dipole-like distributed modes. At frequencies near 34,272 $\mathrm{MHz}$ they are not confined to a given cavity, but rather their fields may penetrate into all the RFcavities, the collector, and perhaps even the gun. These modes cannot by themselves interact with the electron beam at 34,272 $\mathrm{MHz}$ because their $m$-number (number of azimuthal variations) is 1 , and they are out of synchronism. However, since they present everywhere, in particular in the collector (whose volume is large, and which is machined with lose tolerances), their frequencies may be not easily predictable because of the inaccuracy in machining. One of these modes may happen in a very close proximity to $34,272 \mathrm{MHz}$, and couple with the working mode $\mathrm{H}_{311} / \mathrm{TE}_{311}$ (e.g. via the waveguides) because in the output cavity the working mode itself has a certain dipole-like distortions to its fields (happening, as described earlier, because of the presence of waveguides). To see the possible harmful effect of this coupling, we first present a hypothetical case when the dipole modes are far from the working frequency [see Fig. 18]. One observes that the field of $\mathrm{H}_{311} / \mathrm{TE}_{311}$ is not distorted. Simulations also show that the frequency of $\mathrm{H}_{311} / \mathrm{TE}_{311}$ and its $Q$-factor are as expected to be for a pure mode (in a given cavity geometry). Should however, one of the (hypothetical) dipole mode happen to have a very little de-tuning from 34,272 MHz, it couples with the working mode and two hybrids are formed (as usual), with one resembling the working mode but having different frequency, $Q$-factor and field profiles. Thus this hybrid [see Fig. 19] cannot interact anymore with the electron beam.

Two solutions to this problem were considered, and are briefly described here: (a) using choke cavities on either side of the output cavity to prohibit dipole modes from penetrating to the output cavity at $34,272 \mathrm{MHz}$; or (b) removing the unwanted dipole modes by manufacturing the collector with an empirically-determined length. Fig. 20 presents an attempt to employ two chokes; each has its length $(L)$ being originally $\lambda / 4$, and the radial elevation $(\mathrm{d} R)$ being originally between $\lambda / 4$ and $\lambda / 2$; these are varied to see if dipole-mode suppression can be achieved. (In a numerical thought experiment, the drift pipe upstream of the cavity \#6 is assumed to be shortened and its length, $\Delta$, is changed to tune/ de-tune the frequencies of the dipole modes.) 


\section{Omega-P, Inc.}

Phase II proposal submitted following Phase I DoE SBIR grant DE-SC000 4497

in response to DoE SBIR/STTR 2010 Solicitation

Topic 65c: RADIO FREQUENCY POWER SOURCES AND COMPONENTS

RF Sources for High-Gradient Accelerator Research

\section{MODIFIED MAGNICON FOR HIGH-GRADIENT ACCELERATOR R\&D}

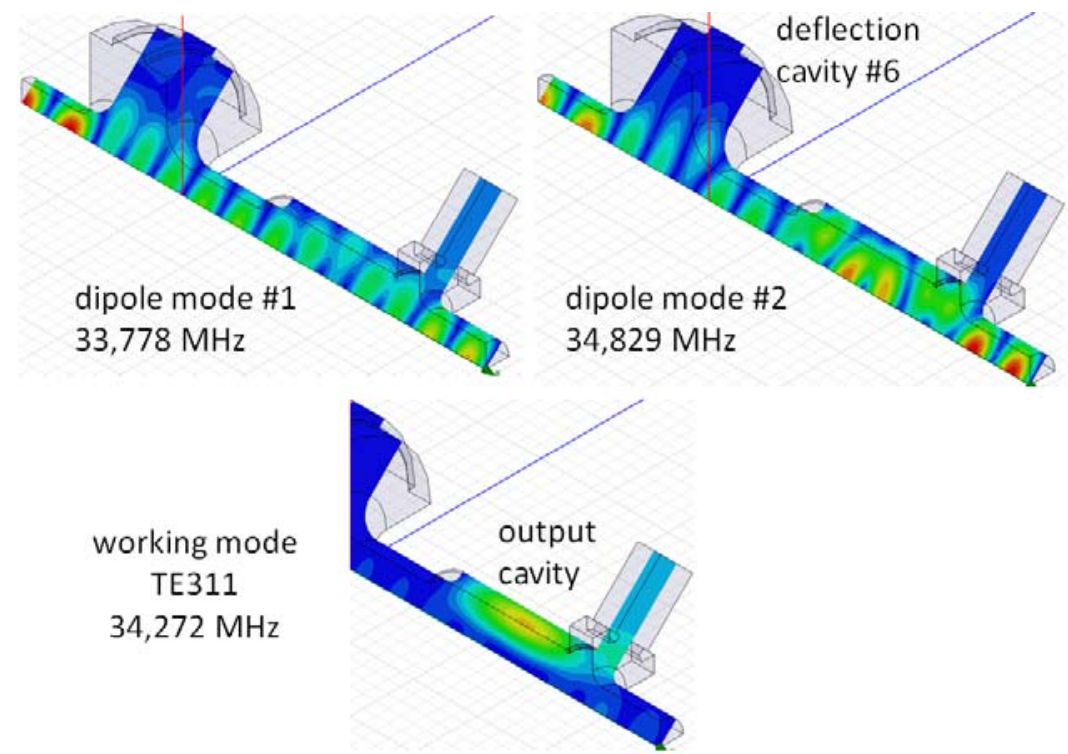

Fig. 18. Field maps (HFSS, complex magnitude of the total electric field) of two dipole modes, one being below and the other being above $34,272 \mathrm{MHz}$, where the working mode exists.
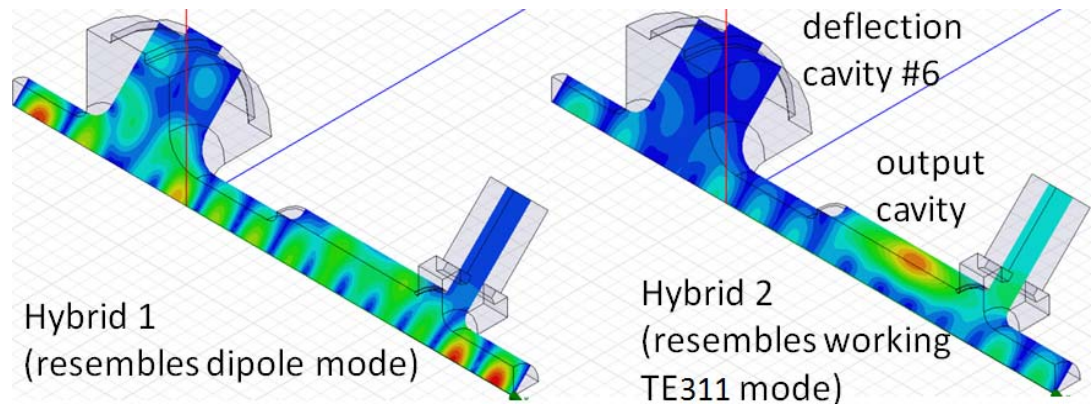

Fig. 19. Field maps (HFSS, complex magnitude of the total electric field) of two hybrid modes formed when a dipole modes couples with the working mode.

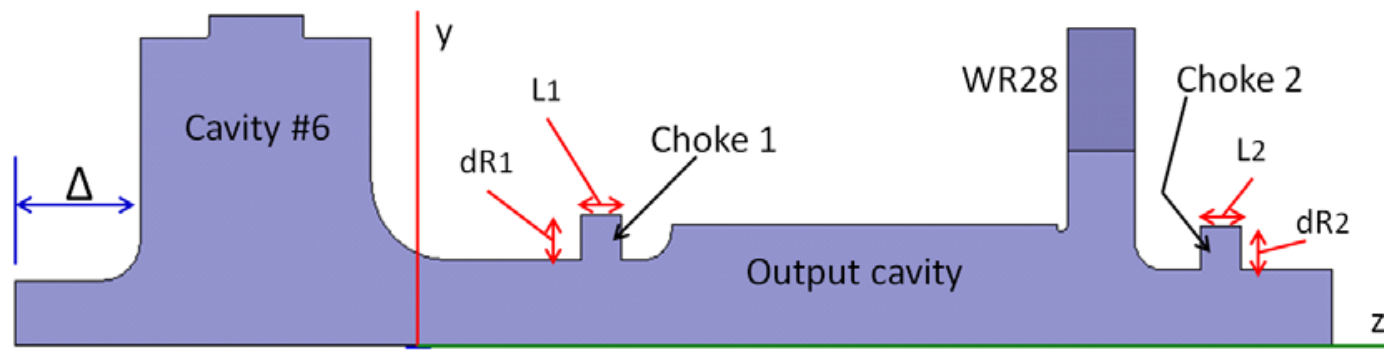

Fig. 20. Chokes to attempt to prevent dipole modes from penetrating to the output cavity when their frequencies are close to $34,272 \mathrm{MHz}$, and thus, preventing them from coupling with the working $\mathrm{H}_{311} / \mathrm{TE}_{311}$ mode localized in the output cavity. 
Omega-P, Inc.

Phase II proposal submitted following Phase I DoE SBIR grant DE-SC000 4497

in response to DoE SBIR/STTR 2010 Solicitation

Topic 65c: RADIO FREQUENCY POWER SOURCES AND COMPONENTS

$R F$ Sources for High-Gradient Accelerator Research

MODIFIED MAGNICON FOR HIGH-GRADIENT ACCELERATOR R\&D
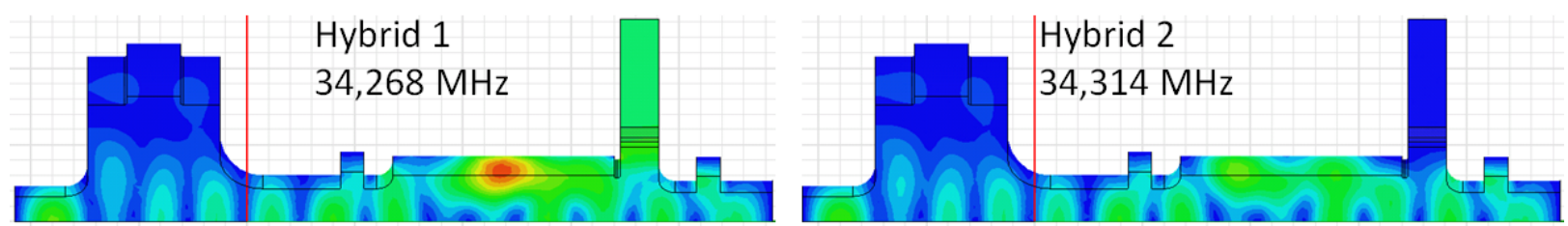

Fig. 21. Example of two resulting hybrid modes resulting from the coupling between the dipole mode and the working mode; the chokes cannot prevent the coupling because of their narrow bandwidth as compared to the bandwidth of the working mode $(\sim 170 \mathrm{MHz})$.

The working mode has its bandwidth of $\sim 170 \mathrm{MHz}$, and the dipole modes change their bandwidth substantially because they may or may not be coupled well with the waveguides connected to the output cavity. The chokes, however, being narrow bandwidth devices do not appear to succeed in preventing any of the dipole modes from coupling with the working mode, if this dipole mode should happen to be tuned sufficiently close to $34,272 \mathrm{MHz}$. This is demonstrated by Fig.21, where two resulting hybrid modes are depicted.

Further study of the approach of employing chokes is advisable. For instance, instead of one choke on each side of the output cavity, two can be used, detuned so that to give a wider bandwidth. However, it also seems that having so many choke-cavities will result in unnecessary complex brazing joints between the deflection cavity \#6 and the output cavity, and between the output cavity and the collector as well. To summarize, employing chokes has so far not been shown to be an appealing solution here. But a second strategy to deal with distributed dipole modes (if any) has also been devised, as follows.

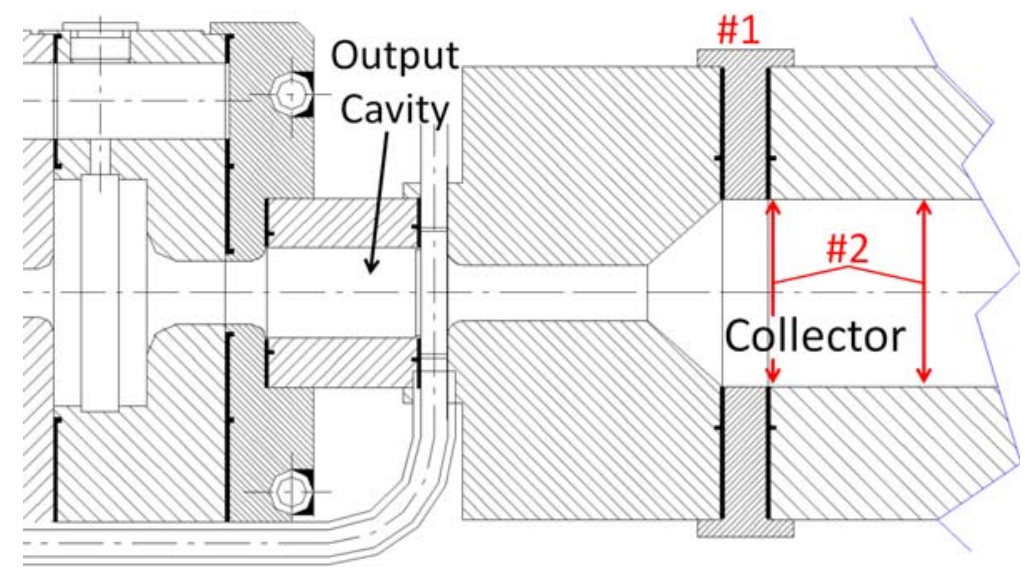

Fig. 22. Possible ways to modify the collector design to enable de-tuning of the dipole modes if needed (see description in the text). 
Omega-P, Inc.

Phase II proposal submitted following Phase I DoE SBIR grant DE-SC000 4497

in response to DoE SBIR/STTR 2010 Solicitation

Topic 65c: RADIO FREQUENCY POWER SOURCES AND COMPONENTS

$R F$ Sources for High-Gradient Accelerator Research

MODIFIED MAGNICON FOR HIGH-GRADIENT ACCELERATOR R\&D

It can be noted that with the dipole modes being distributed ones, having one in close proximity of the operating frequency $(34,272 \mathrm{MHz})$ is a highly unlikely event. However, should this occur, another far more practical and robust solution can be employed, such as to remove the presence of a dipole mode (if any) from the vicinity of working frequency at the stage of manufacturing before brazing, as follows:

(1) Tune the working $\mathrm{H}_{311} / \mathrm{TE}_{311}$ mode in a stand-alone output cavity to $34,272 \mathrm{MHz}$ and $Q$ of 200 .

(2) Assemble the entire RF-cavity chain and the collector, and measure the frequency and $Q$-factor of the working mode.

(3) If the measured values differ from that of the stand-alone cavity, a dipole mode is near $34,272 \mathrm{MHz}$, and must be de-tuned. Here it is useful to note that the $Q$-factor is a good and very sensitive measure if the working mode couples with any parasitic mode.

(4) Remove the collector and adjust its dimensions (e.g. the length of the ring-like insertion in pos \#1 in Fig. 22, or skim the diameter in the beginning of the collector body (pos \#2).

(5) Re-attach the collector and repeat the steps \#1 through 4 until the measured frequency and $Q$-factor for the working mode are those of the standalone cavity.

(6) Perform brazing (of the collector) only after steps \#1- 5 are accomplished.

It is this latter presented solution that constitutes our current working plan to suppress unwanted dipole modes (if any).

\section{Measurement of loaded $Q$-factor and spectrum of modes using a cavity mockup}

An aluminum mockup of the output cavity was built and used to measure the loaded $Q$ factor, as well as to study the mode spectrum. Details are shown in Fig. 23. 


\section{Omega-P, Inc.}

Phase II proposal submitted following Phase I DoE SBIR grant DE-SC000 4497 in response to DoE SBIR/STTR 2010 Solicitation Topic 65c: RADIO FREQUENCY POWER SOURCES AND COMPONENTS $R F$ Sources for High-Gradient Accelerator Research

\section{MODIFIED MAGNICON FOR HIGH-GRADIENT ACCELERATOR R\&D}
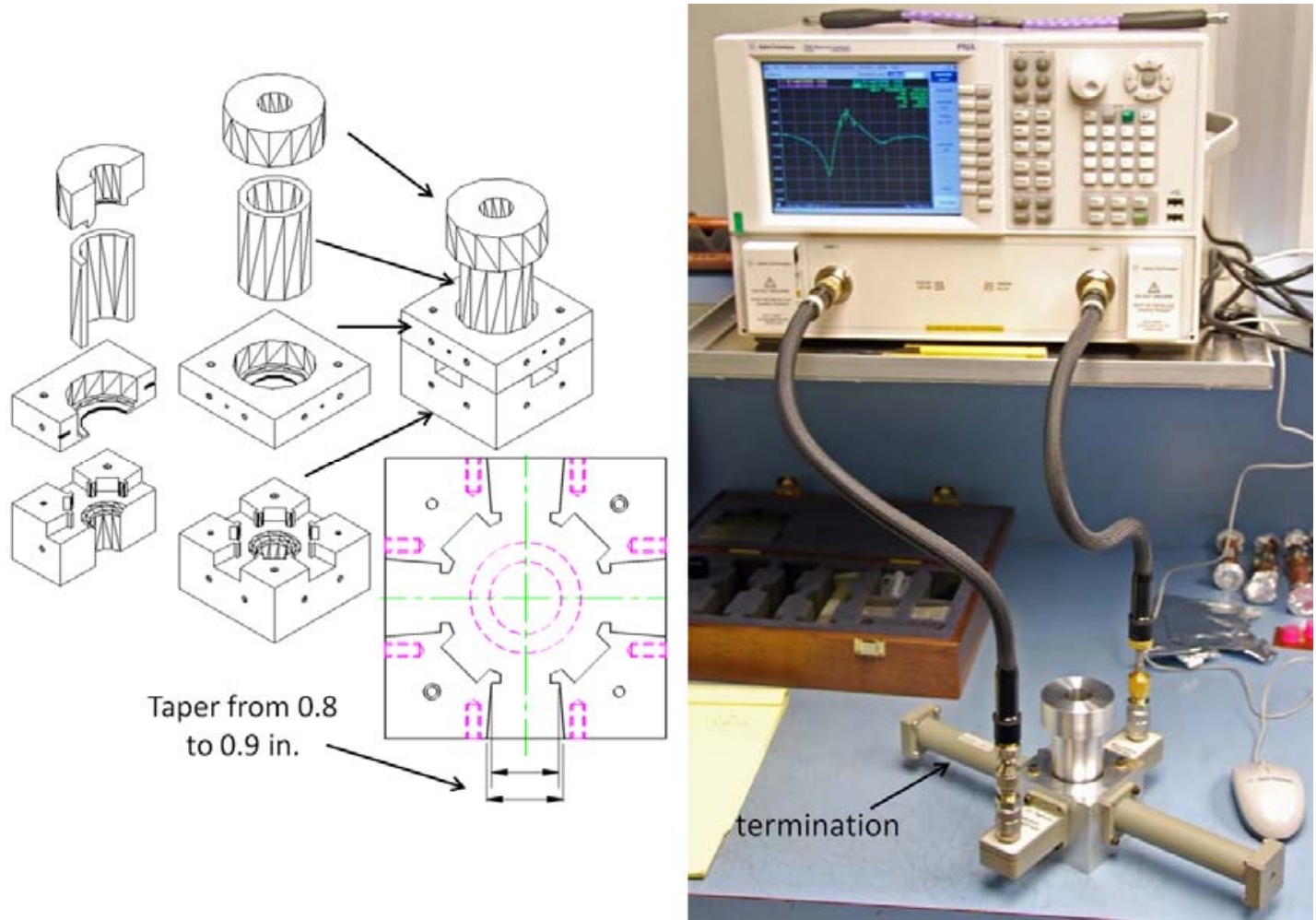

Fig. 23. 3-D drawings of the cavity mockup (left) and a photo showing the mockup connected to an Agilent vector network analyzer, model \# PNA E8364C, recently acquired by the Yale Ka-

Band Test Facility. The mockup is scaled to X-band with a scaling coefficient of 2.857 (see explanations in text).

The mockup is scaled from Ka-band to X-band. The scaling coefficient is 2.857, which is the ratio of the height of WR90 [0.40 in.] to the height of WR28 [0.14 in.]. This is because the length of the $2^{\text {nd }}$ chamber is that of the height of WR2 8 waveguide. To have the mockup scaled correctly, the length of the scaled $2^{\text {nd }}$ chamber must be that of WR90 waveguide. The width of WR28 cannot be scaled with the same coefficient to the width of WR90; thus, there is a slight taper which follows each waveguide port aperture to match to the width of WR90.

Given the fact that this mockup was built while the work to refine the cavity design was still in progress, the mockup sizes do not quite reproduce the sizes presented in Table III (but multiplied by 2.857$)$. The sizes $(\mathrm{mm})$ that are different are: $\mathrm{L}=61.47, \mathrm{R}_{\text {chamber } 1}=17.31, \mathrm{R}_{\text {chamber2 }}$ $=23.63, \mathrm{R}_{\text {iris }}=16.49$ and $\mathrm{R}_{2}=\mathrm{R}_{3}=0.859$. Relative simplicity of the mockup and availability of machining tools dictated the choice of machining accuracy to be $100 \mu \mathrm{m}$. To have a valid 
Omega-P, Inc.

Phase II proposal submitted following Phase I DoE SBIR grant DE-SC000 4497

in response to DoE SBIR/STTR 2010 Solicitation

Topic 65c: RADIO FREQUENCY POWER SOURCES AND COMPONENTS

$R F$ Sources for High-Gradient Accelerator Research

MODIFIED MAGNICON FOR HIGH-GRADIENT ACCELERATOR R\&D

comparison between the predictions and measurements, new numerical simulations were done for these adjusted sizes.

A digitally uploaded snapshot of a network analyzer trace showing the working $\mathrm{TE}_{311} / \mathrm{H}_{311}$ mode, and also the nearby $\mathrm{TE}_{312} / \mathrm{H}_{312}$ mode is presented in Fig. 24. A satisfactory agreement between the measured and predicted mode frequencies and $Q$-factors has been found. The measured loaded $Q$-factor for the working mode is 226 , which compares well with the computed value of 220; the measured $Q$-factor for the $\mathrm{TE}_{312}$ mode is $65-70$, and also compares well with the computed value of 71 . The measured frequency for the working mode is 11,871 $\mathrm{MHz}$, which differs from the computed value of $11,923 \mathrm{MHz}$ by $-72 \mathrm{MHz}$. The measured frequency for the $\mathrm{TE}_{312}$ mode is $12,384 \mathrm{MHz}$ which differs from the computedvalue of 12,426 $\mathrm{MHz}$ by $-35 \mathrm{MHz}$. The frequency seperation betweenthe $\mathrm{TE}_{311}$ and $\mathrm{TE}_{312}$ modes is measured to be $513 \mathrm{MHz}$, while the computed value is $503 \mathrm{MHz}$. All the cited values are within the accuracy arising from the specified machining accuracy.

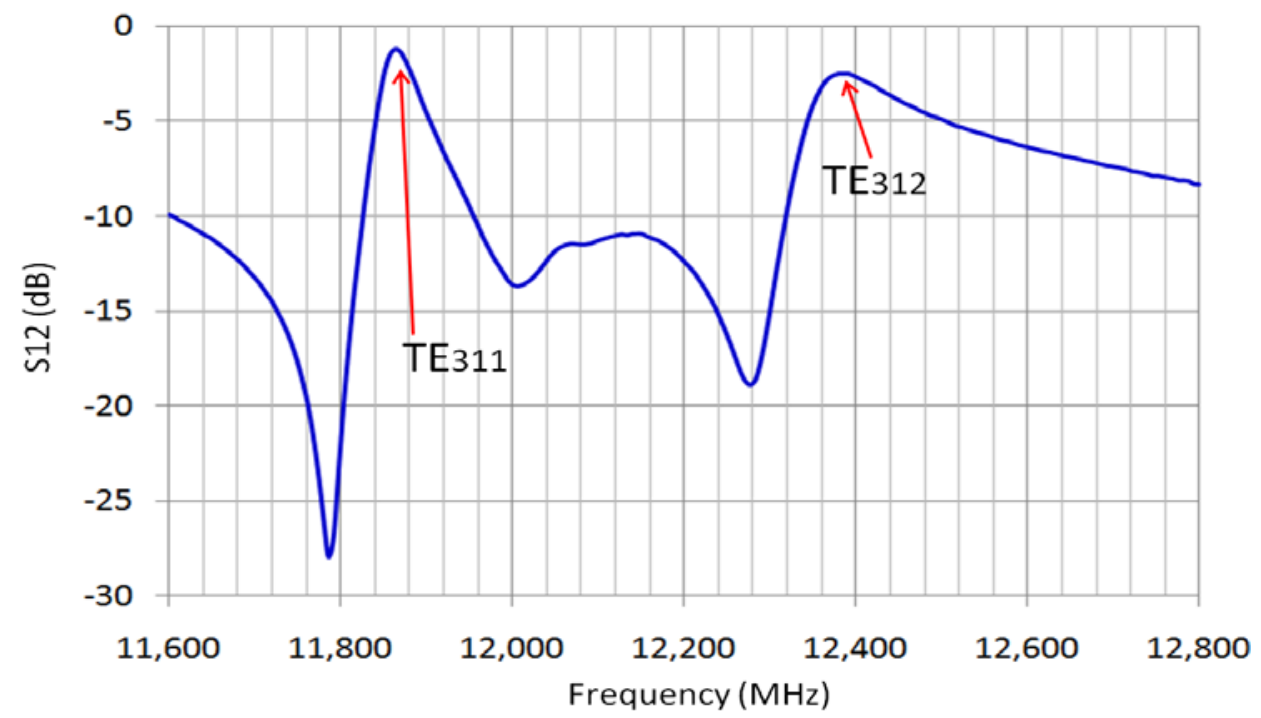

Fig. 24. Digitally uploaded snapshot of a network analyzer trace showing two of the modes.

\section{IIc. Modifications to the system of deflection cavities}

The deflection cavity system will have its first five cavities to be of the same design as the present tube. However, these cavities must be detuned differently. Cavity \#6 (penultimate cavity) will be of a new design, and also detuned differently than the present penultimate cavity. The detuning is done from $11.424 \mathrm{GHz}$, and the numbers are presented in Table 3.1. 
Omega-P, Inc.

Phase II proposal submitted following Phase I DoE SBIR grant DE-SC000 4497

in response to DoE SBIR/STTR 2010 Solicitation

Topic 65c: RADIO FREQUENCY POWER SOURCES AND COMPONENTS

$R F$ Sources for High-Gradient Accelerator Research

MODIFIED MAGNICON FOR HIGH-GRADIENT ACCELERATOR R\&D

Table VI. Detuning of deflection cavities (MHz) from $11.424 \mathrm{GHz}$

\begin{tabular}{|c|c|c|c|c|c|}
\hline Cavity \#1 & $\# 2$ & $\# 3$ & $\# 4$ & $\# 5$ & $\# 6$ \\
\hline $41.5 \mathrm{MHz}$ & 53.4 & 39.6 & 52.3 & 43.7 & 25.1 \\
\hline
\end{tabular}

With this detuning, the deflection system is well optimized to work with the $450 \mathrm{kV}$ electron beam, at a current of $187 \mathrm{~A}$. However, since the exit pipe radius is presently $3.75 \mathrm{~mm}$, the drive power would not be able to exceed $\sim 125$ Watts; for otherwise the deflection angle would be too large and the outermost electrons would hit the pipe walls [see Figs. 25 and 26]. This in turn would limit the efficiency and output power [Fig. 27]. Clearly, the last drift pipe radius (which is the exit drift pipe of cavity \#6) must be increased so as not to impose any limits. Making the radius $4.25 \mathrm{~mm}$ appears to be a correct choice [see e.g. Fig. 25 again].
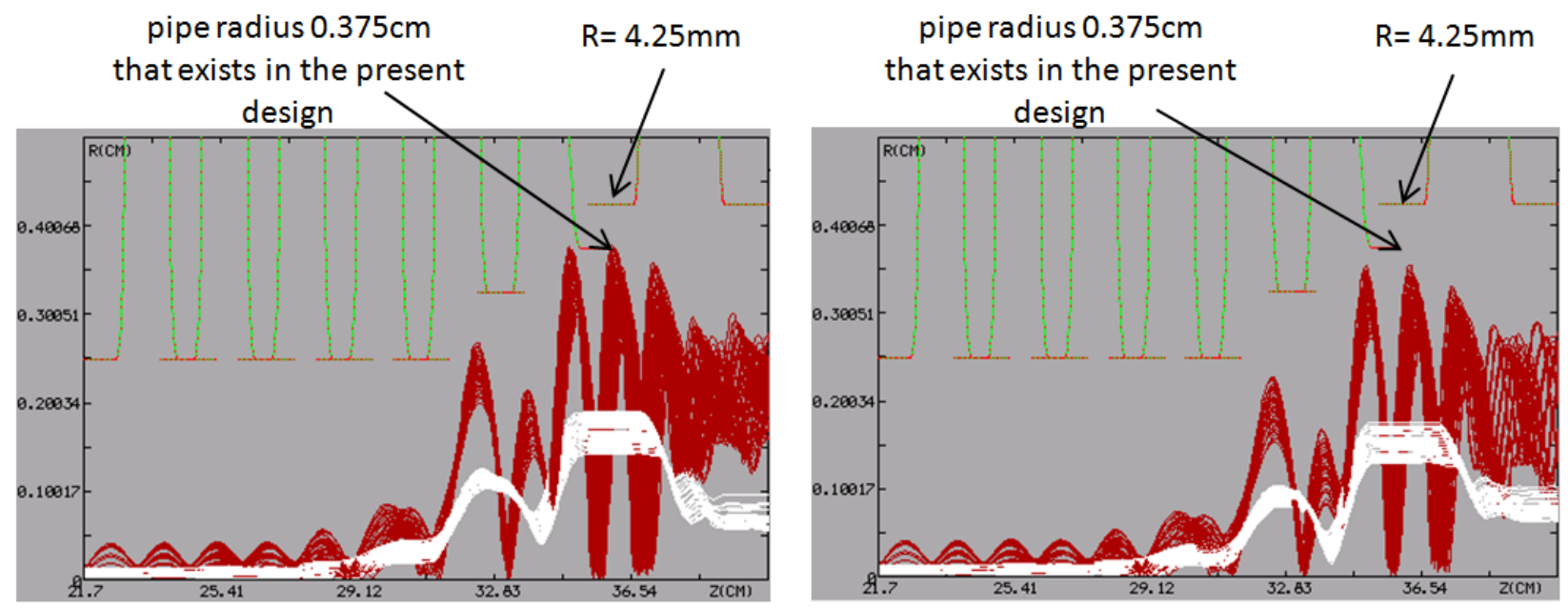

Fig. 25. (left) In this graphical output from MGC v.4.15, the red curves are trajectories of the different parts of the e-beam. With the deflection system re-optimized the outermost electrons can hit the drift pipe at the exit from the last deflection cavity. This example is for $200 \mathrm{~W}$ of input drive power [at 11.427.6 GHz]. (right) With the deflection system re-optimized, but operating at a low input power level of $\sim 125$ Watts, the outermost electrons do not hit the drift pipe. The penalty would be a diminution in efficiency and output power (from $\sim 27$ to $21 \mathrm{MW}$ ). 


\section{Omega-P, Inc.}

Phase II proposal submitted following Phase I DoE SBIR grant DE-SC000 4497

in response to DoE SBIR/STTR 2010 Solicitation

Topic 65c: RADIO FREQUENCY POWER SOURCES AND COMPONENTS

$R F$ Sources for High-Gradient Accelerator Research

\section{MODIFIED MAGNICON FOR HIGH-GRADIENT ACCELERATOR R\&D}
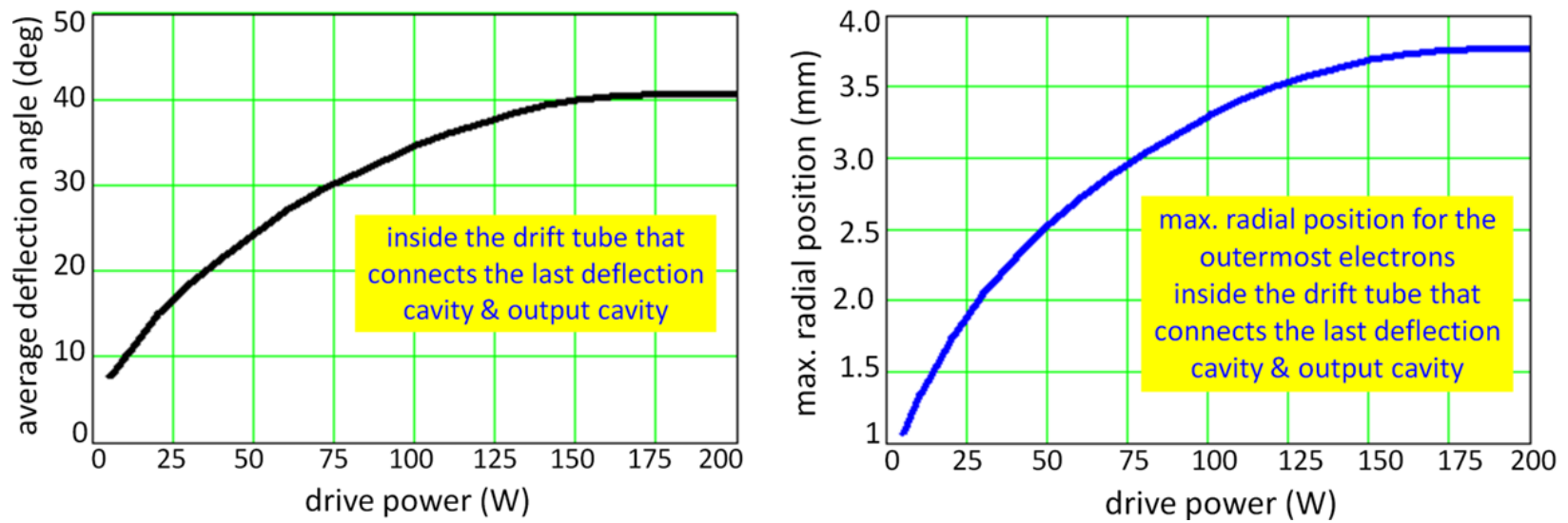

Fig. 26. The plot on the right-hand side shows that, at a drive power in excess of $125 \mathrm{~W}$, the outermost electrons reach a radius of $3.75 \mathrm{~mm}$, because they are strongly deflected.

Consequently they hit the wall of the last drift pipe, whose radius presently is $3.75 \mathrm{~mm}$ (see Fig. 25). Clearly, the last drift pipe should be increased in radius.

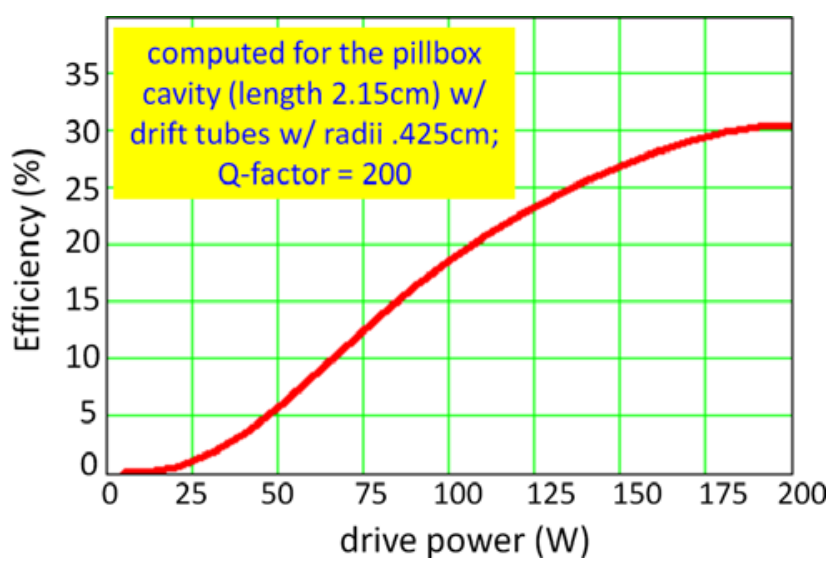

Fig. 27. This example serves to remind that the interaction efficiency (and thus power) is much less if one operates at $125 \mathrm{~W}$ of drive power - the present limitation because the last cavity would have its exit pipe radius $3.75 \mathrm{~mm}$. Increasing this radius to $4.25 \mathrm{~mm}$ would allow operating at $\sim 200 \mathrm{~W}$ of drive power.

\section{New penultimate cavity (cavity \#6)}

Here design of the $6^{\text {th }}$ deflection cavity with an exit drift tube radius of $4.25 \mathrm{~mm}$ is described. Three issues have been addressed: (a) one must demonstrate that the cavity with a new drift pipe may deliver the same or nearly the same deflection angle at its exit; (b) one must demonstrate that the spectrum of modes in the vicinity of the operating frequency is clean enough to isolate the working mode reliably, and thus to tune it accurately; and lastly (c) it must be demonstrated that the dimensions of the cavity can be such that High Order Modes [HOMs] 
Omega- $P$, Inc.

Phase II proposal submitted following Phase I DoE SBIR grant DE-SC000 4497

in response to DoE SBIR/STTR 2010 Solicitation

Topic 65c: RADIO FREQUENCY POWER SOURCES AND COMPONENTS

$R F$ Sources for High-Gradient Accelerator Research

MODIFIED MAGNICON FOR HIGH-GRADIENT ACCELERATOR R\&D

are not excited. The configuration of the $6^{\text {th }}$ cavity is shown in Fig. 28, and the parameters/sizes are given in Table VII.
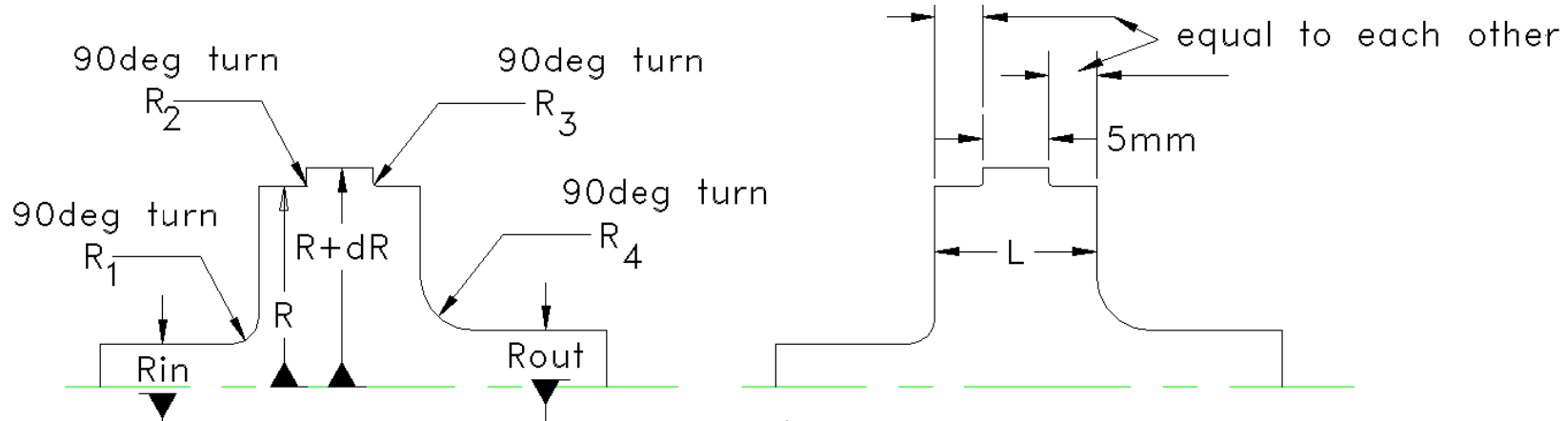

Fig. 28. The configuration of the $6^{\text {th }}$ cavity [see Table VII for the sizes]

Table VII. Redesigned $6^{\text {th }}$ cavity

\begin{tabular}{|c|c|}
\hline working mode & $\mathrm{E}_{110} / \mathrm{TM}_{110}$ \\
\hline operational frequency & $11,449.1 \mathrm{MHz}$ \\
\hline$Q$-factor (expected) & $\sim 9150$ \\
\hline $\mathrm{R}, \mathrm{mm}$ & 15.200 \\
\hline $\mathrm{dR}, \mathrm{mm}$ & 1.1656 [expected to be after tuning] \\
\hline $\mathrm{L}, \mathrm{mm}$ & 12.275 \\
\hline $\mathrm{R}_{1}, \mathrm{~mm}$ & 2 \\
\hline $\mathrm{R}_{2}, \mathrm{~mm}$ & 0.25 \\
\hline $\mathrm{R}_{3}, \mathrm{~mm}$ & 0.25 \\
\hline $\mathrm{R}_{4}, \mathrm{~mm}$ & 4 \\
\hline $\mathrm{R}, \mathrm{mm}$ & 3.25 \\
\hline $\mathrm{Rout}, \mathrm{mm}$ & 4.25 \\
\hline tolerances on $\mathrm{R}, \mathrm{R}_{2}$ and $\mathrm{R}_{3}$ & $-10 /+10$ microns \\
\hline tolerances on $\mathrm{R}_{1}$ and $\mathrm{R}_{4}$ & $-20 /+20$ microns \\
\hline tolerances on the length, $\mathrm{L}$ & $-10 /+20$ microns \\
\hline tolerances when enlarging the groove & $-/+3$ microns \\
\hline size, dR & {$[$ to have tuning accuracy $-/+1 \mathrm{MHz}]$} \\
\hline
\end{tabular}

Note that the $6^{\text {th }}$ cavity must be tuned to $11,449 \mathrm{MHz}$ by adjusting the groove size, $\mathrm{dR}$. The dRvalues shown in the tables are the expected to be as given by results of HFSS simulations. When $\mathrm{dR}$ is increased, the machining accuracy must be $3 \mu \mathrm{m}$ to guaranty a tuning accuracy of $\sim 1 \mathrm{MHz}$. 


\section{Omega-P, Inc.}

Phase II proposal submitted following Phase I DoE SBIR grant DE-SC000 4497

in response to DoE SBIR/STTR 2010 Solicitation

Topic 65c: RADIO FREQUENCY POWER SOURCES AND COMPONENTS

$R F$ Sources for High-Gradient Accelerator Research

MODIFIED MAGNICON FOR HIGH-GRADIENT ACCELERATOR R\&D

\section{Dynamics of the e-beam in the new $6^{\text {th }}$ cavity}

To demonstrate that the drift tube radius at the exit of the $6^{\text {th }}$ deflection cavity can be increased up to $4.25 \mathrm{~mm}$, it is instructive to compare the performances of the deflection system with the already available design for the $6^{\text {th }}$ cavity (that which has an exit drift pipe radius of $3.75 \mathrm{~mm}$ ), and the re-designed version with the exit drift tube whose radius is $4.25 \mathrm{~mm}$. The measure of performances of the deflection system is as usual the orbit angle at its exit, since the efficiency of a magnicon type device is proportional to the square of the sine of the angle which the e-beam has when it emerges from the deflection system.

The deflection angle after the $6^{\text {th }}$ cavity for the old cavity design and the new design presented in Table 3.2 is plotted $v s$ the drive power in Fig. 29. It is seen that the performances are nearly the same for both versions, thus proving that one may use the cavity with the exit drift pipe radius of $4.25 \mathrm{~mm}$. One notes, however, that this adjusted cavity will allow operating above $125 \mathrm{~W}$ of drive power, as the e-beam will pass through the drift tube without hitting the walls.

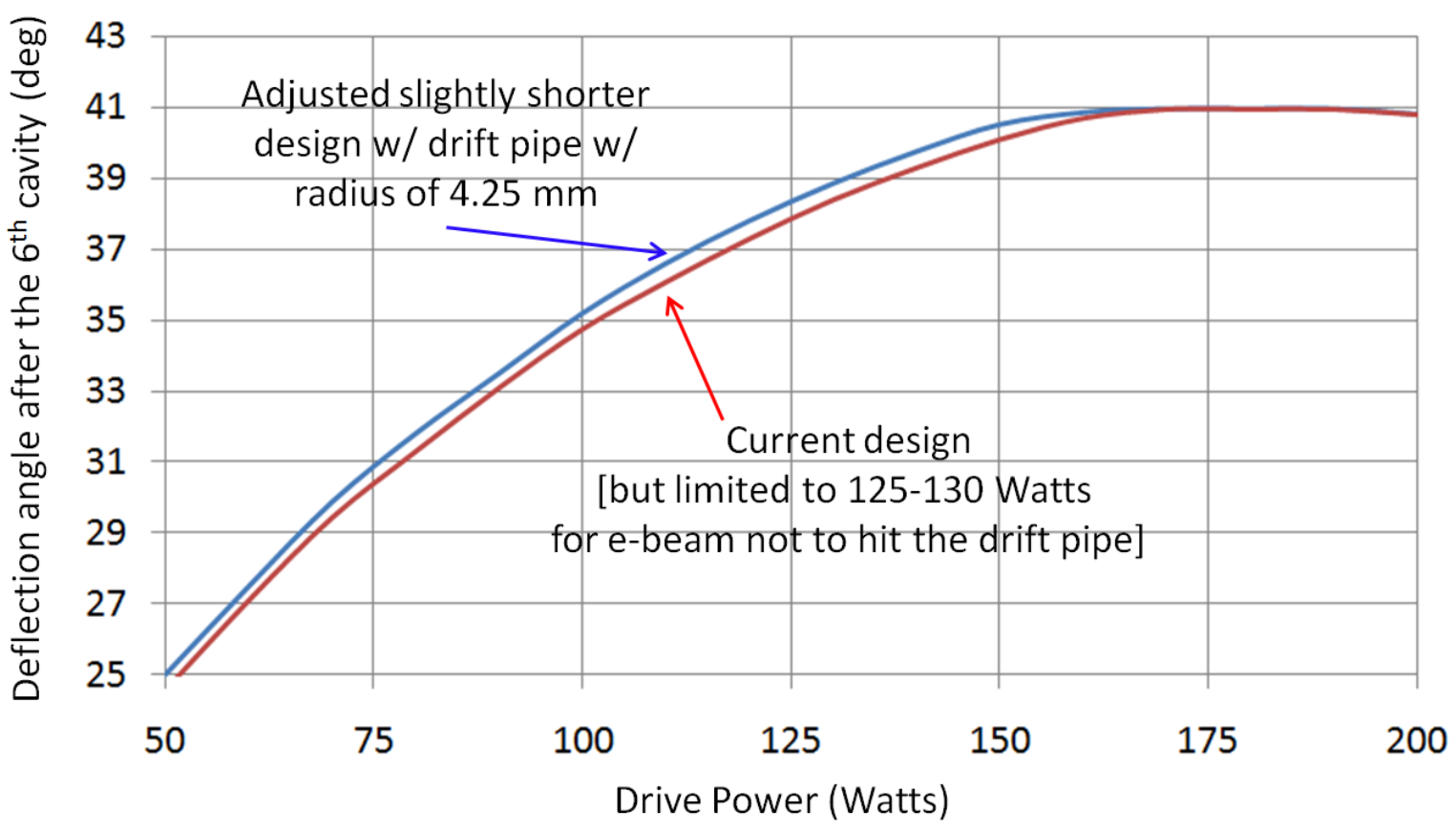

Fig. 29. The deflection angle (degree) as a measure of performance of the deflection system plotted vs the applied drive power (W) for the old cavity design and the new design as given in

Table 3.2. One observes that the performances are nearly the same for both versions, notwithstanding the limit of $125 \mathrm{~W}$ for the smaller drift pipe. 
Omega-P, Inc.

Phase II proposal submitted following Phase I DoE SBIR grant DE-SC000 4497

in response to DoE SBIR/STTR 2010 Solicitation

Topic 65c: RADIO FREQUENCY POWER SOURCES AND COMPONENTS

$R F$ Sources for High-Gradient Accelerator Research

MODIFIED MAGNICON FOR HIGH-GRADIENT ACCELERATOR R\&D

Spectrum of the $6^{\text {th }}$ cavity in the vicinity of the operational frequency

The spectrum of the $6^{\text {th }}$ cavity in the vicinity of the operating frequency $11.4 \mathrm{GHz}$ is remarkably clean, as seen in Figs 30. Examining the information presented in the field plots, one concludes that there are no modes which dwell in the proximity of the operational frequency to affect the e-beam dynamics or to interfere with frequency $/ Q$-factor measurements when the cavity operating mode $\mathrm{E}_{110} / \mathrm{TM}_{110}$ is tuned to the required $11,449.1 \mathrm{MHz}$.

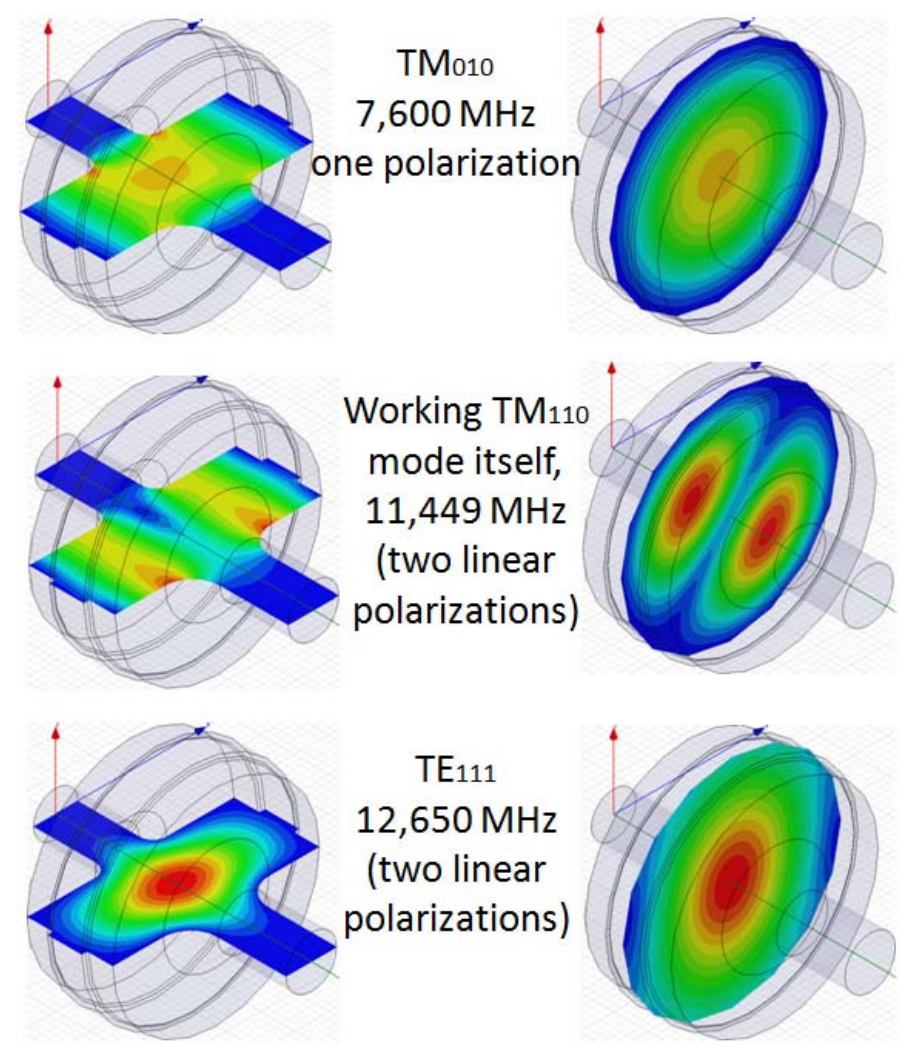

Fig. 30. (Top) The closest mode $\left(\mathrm{E}_{010} / \mathrm{TM}_{010}\right)$ from below in the $6^{\text {th }}$ deflection cavity (one polarization presents) dwells at $\sim 7,600 \mathrm{MHz}$. This mode should not interfere when the cavity is tuned. (Center) The working mode $\left(\mathrm{E}_{110} / \mathrm{TM}_{110}\right)$ which is in the $6^{\text {th }}$ deflection cavity (two linear polarization present) must be tuned to $11,449 \mathrm{MHz}$ as suggested by MGC simulations for an efficient amplification of the deflection angle. (Bottom) The closest mode $\left(\mathrm{H}_{111} / \mathrm{TE}_{111}\right)$ from above in the $6^{\text {th }}$ deflection cavity (two linear polarization present) dwells at a frequency of $\sim 12,650 \mathrm{MHz}$. Consequently, this mode should neither affect the beam dynamics; nor should it interfere when the cavity is tuned. (HFSS v12 simulations; complex magnitude of the total electric field is plotted.) 


\section{Omega-P, Inc.}

Phase II proposal submitted following Phase I DoE SBIR grant DE-SC000 4497 in response to DoE SBIR/STTR 2010 Solicitation

Topic 65c: RADIO FREQUENCY POWER SOURCES AND COMPONENTS

$R F$ Sources for High-Gradient Accelerator Research

MODIFIED MAGNICON FOR HIGH-GRADIENT ACCELERATOR R\&D

\section{HOMs in the $6^{\text {th }}$ cavity: foreword}

In a magnicon cavity the HOMs with azimuthal indices $m$, dwelling in the vicinity of the frequencies $f_{\mathrm{m}}=m \times 11,424 \mathrm{MHz}$ may interact efficiently with the electron beam if they are not detuned sufficiently. An example below demonstrates this. Among other HOMs, there is a mode normally known as $\mathrm{E}_{322} / \mathrm{TM}_{322}$ with $m=3$, situated close to the frequency $f_{3}=34,272 \mathrm{MHz}$ which satisfies the rule $f_{3}=3 \times 11,424 \mathrm{MHz}$; of course, in the version as given in Table VII it is detuned sufficiently. However, in our thought experiment we can place it as close to $32,272 \mathrm{MHz}$ as desired to see what sort of effects on the beam dynamics and the performance of the deflection system the lack of detuning might induce. The simulations can be performed by using CLANS2/ MGC supported by Axif9/Decod addons. The $6^{\text {th }}$ cavity in this simulation is assumed to have two modes being excited, namely the working mode $\mathrm{E}_{110}$ and the parasitic mode $\mathrm{E}_{322}$. As usual there is also an output cavity attached behind to see what sort of efficiency one might get. Fig. 31 shows that a significant power by itself may go to a parasitic HOM if detuning is small [detuning is defined as the parasitic HOM frequency minus the value of the $m^{\text {th }}$ harmonic of $11,424 \mathrm{MHz}]$; combined with the fact that HOM have large $Q$-factors $(\sim 20,000)$ this alone may result in severe breakdown in the cavity [here it is the $6^{\text {th }}$ deflection cavity]. Even without taking the breakdowns into consideration one sees that the growing fields of the parasitic HOM strongly affects the e-beam dynamics leading to a significant drop in the deflection angle - see Fig. 32.

The drop in the efficiency can be drastic - it becomes zero at a certain value of detuning [see Fig. 33].

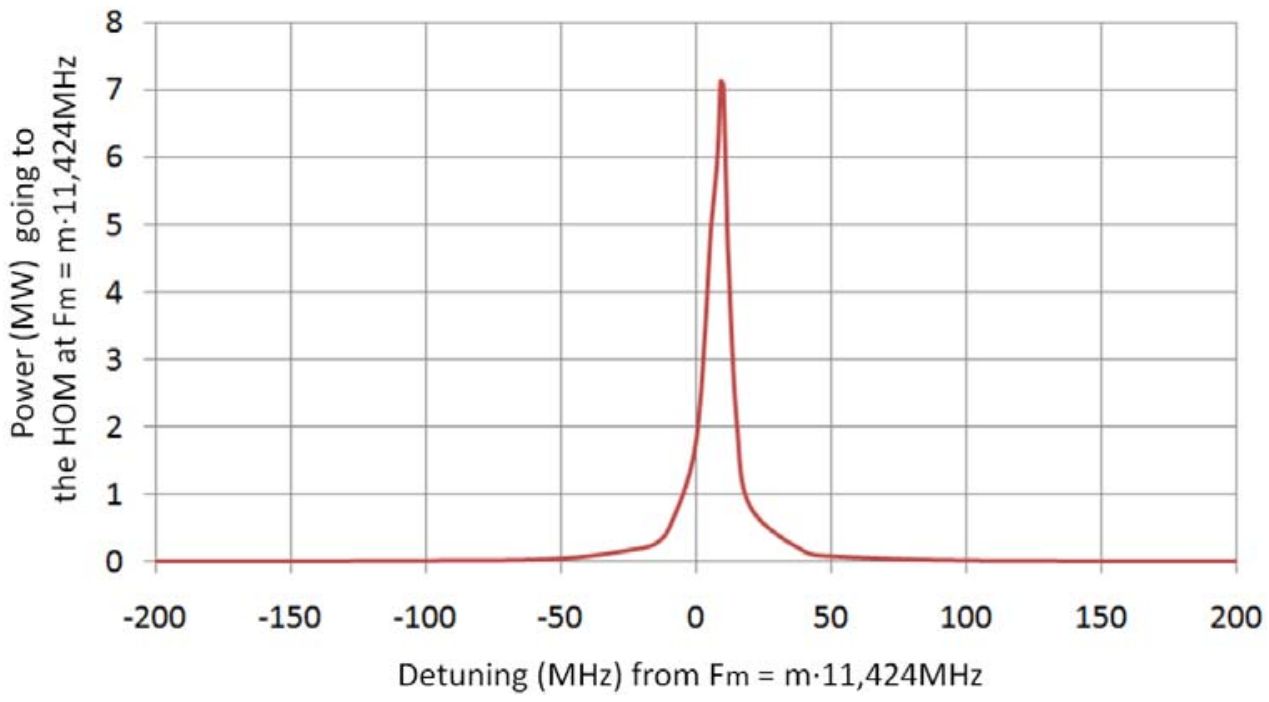

Fig. 31. Power (MW) going to HOM vs. the detuning (MHz) from $f_{\mathrm{m}}=\mathrm{m} \times 11,424 \mathrm{MHz}$. In this example, the parasitic HOM of the $6^{\text {th }}$ deflection cavity is near $3 \times 11,424 \mathrm{GHz}$, and can consume up to $7 \mathrm{MW}$ when the detuning is $9-10 \mathrm{MHz}$. 
Omega-P, Inc.

Phase II proposal submitted following Phase I DoE SBIR grant DE-SC000 4497

in response to DoE SBIR/STTR 2010 Solicitation

Topic 65c: RADIO FREQUENCY POWER SOURCES AND COMPONENTS

$R F$ Sources for High-Gradient Accelerator Research

MODIFIED MAGNICON FOR HIGH-GRADIENT ACCELERATOR R\&D

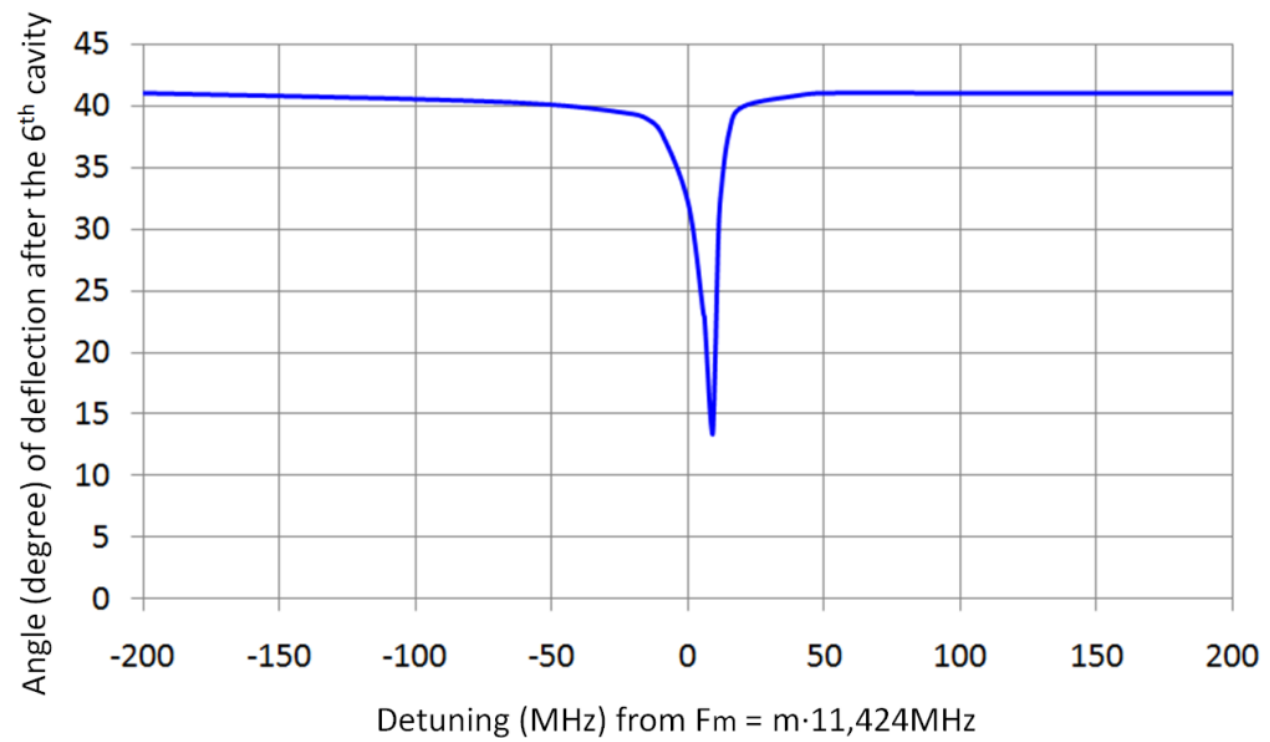

Fig. 32. Deflection angle (deg) after the $6^{\text {th }}$ cavity as affected by the presence of poorly detuned parasitic HOM. The angle may drop by 3-4 times when detuning (plotted here in MHz) is small.

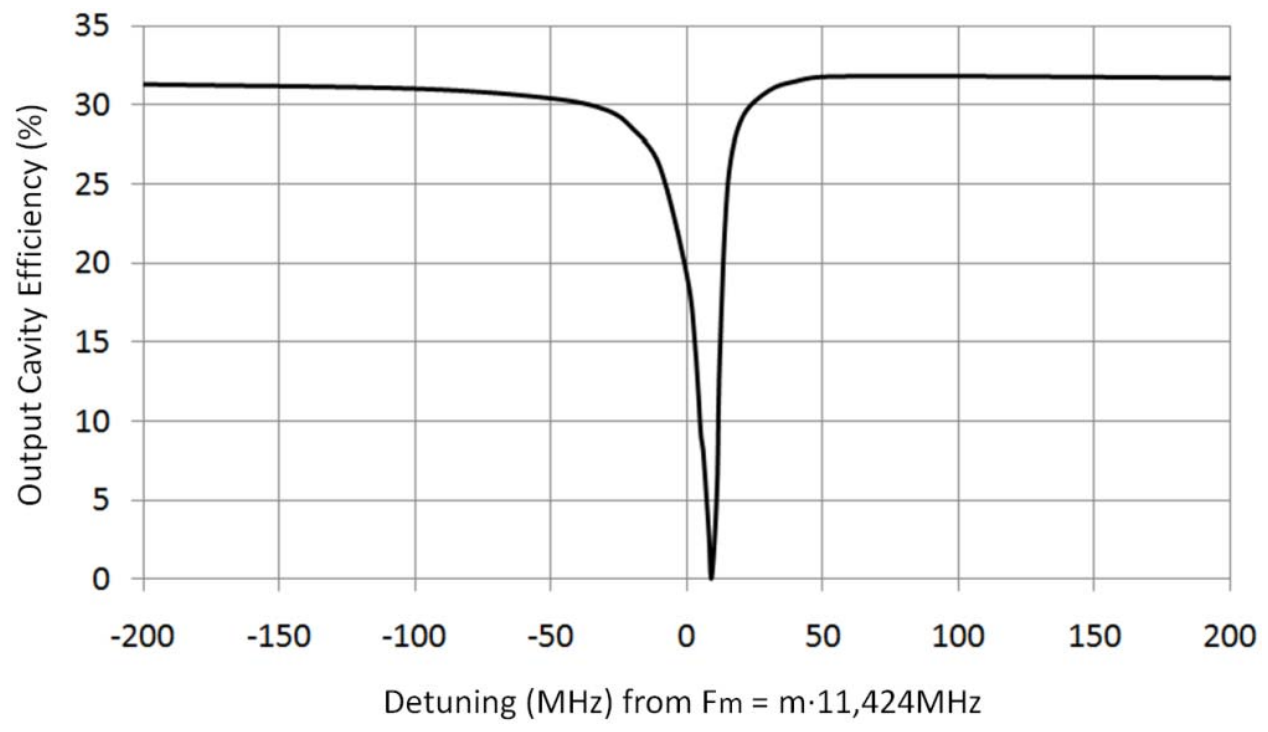

Fig. 33. Efficiency in the output cavity (here it has $Q \sim 200$ ) drops to zero, when the parasitic $\mathrm{HOM}$ in the $6^{\text {th }}$ deflection cavity is weakly detuned. Here the example is for $\mathrm{TM}_{322} / \mathrm{E}_{322} \mathrm{HOM}$, and the efficiency is zero when detuning is $9-10 \mathrm{MHz}$ from the value of the $3^{\text {rd }}$ harmonic frequency $3 \times 11,424 \mathrm{MHz}$.

It was found that, in general, in the $6^{\text {th }}$ deflection cavity, HOMs must be controlled so that they are detuned by $150 \mathrm{MHz}$, or better by $200 \mathrm{MHz}$. Of course, one may point out that in there can 
Omega-P, Inc.

Phase II proposal submitted following Phase I DoE SBIR grant DE-SC000 4497

in response to DoE SBIR/STTR 2010 Solicitation

Topic 65c: RADIO FREQUENCY POWER SOURCES AND COMPONENTS

RF Sources for High-Gradient Accelerator Research

MODIFIED MAGNICON FOR HIGH-GRADIENT ACCELERATOR R\&D

be infinite number of HOMs, since $m$ can be anything from 1 to infinity. Fortunately, the HOMs with $m=10$ and above have low to zero field strength in the region where the e-beam propagates when it procee $\leq$ s down the $6^{\text {th }}$ deflection cavity. Thus below limit our attention to HOMs with $m$ $=1,2, \ldots 9$.

\section{HOMs in the $6^{\text {th }}$ cavity with $2 \leq m \leq 9$}

There is no other mode with $m=1$ except for the working one (which is $\mathrm{E}_{110} / \mathrm{TM}_{110}$ ) in the proximity of $11,424 \mathrm{MHz}$ [and to remind the working mode is tuned to $11,449 \mathrm{MHz}$ to have a good bandwidth for the deflection system]. Thus, we will be looking at the modes with $m=2$, 3 and so on up to 9. The total number of these modes is an appreciable one, namely 22 . They were studied by HFSS, and the plots of their complex magnitude of total electrical field are shown in the series of pictures labeled Fig. 34. To have selectivity for a given $m$-number (number of azimuthal variations), not an entire cavity geometry was inputted in HFSS, but only a sector of it, with the angular dimensions of the sector being $90 \% \mathrm{~m}$ and the boundary condition on the faces of the sector being that of perfect electric wall on one face, and perfect magnetic wall on the other face.
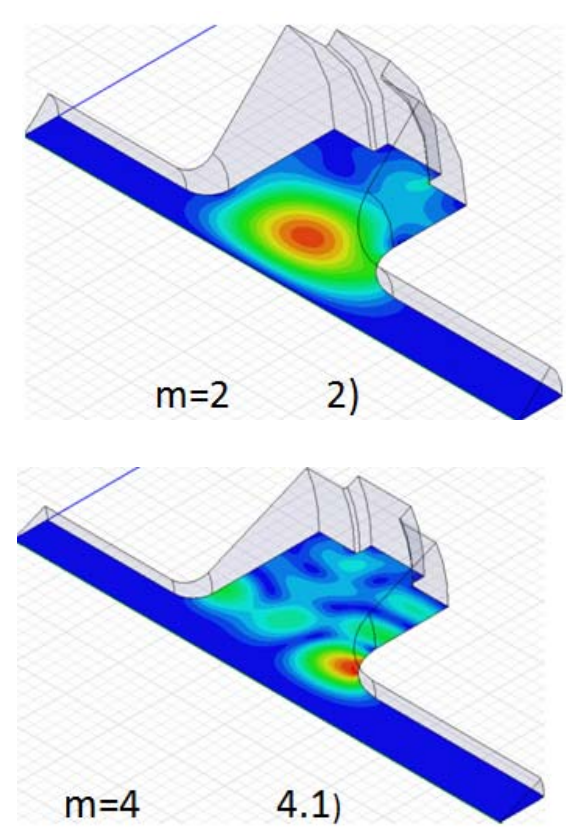
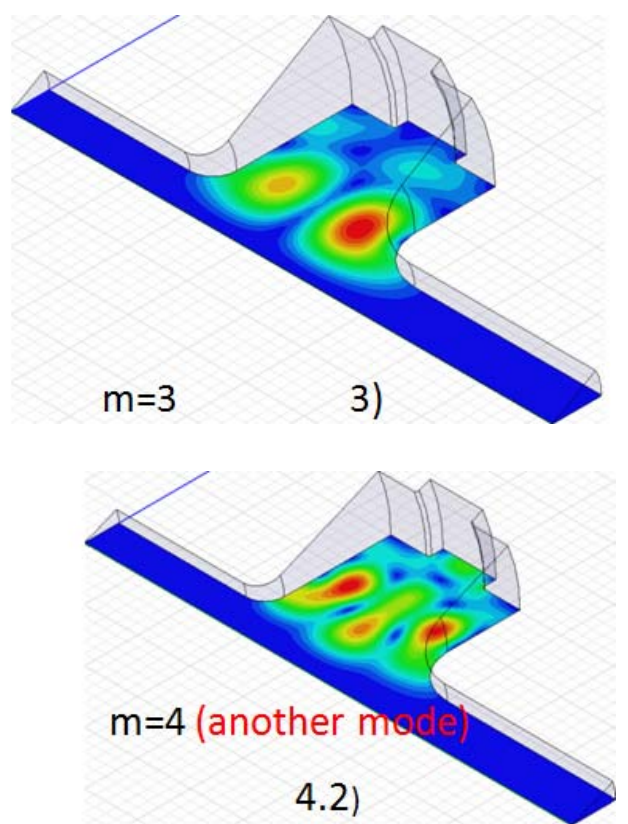

4.2)

Fig. 34. HOMs as simulated by HFSS with $2 \leq \mathrm{m} \leq 9$

. For clarity we start the plot enumeration with 2 , corresponding to the azimuthal index $m$. (continued on next page) 


\section{Omega-P, Inc.}

Phase II proposal submitted following Phase I DoE SBIR grant DE-SC000 4497 in response to DoE SBIR/STTR 2010 Solicitation

Topic 65c: RADIO FREQUENCY POWER SOURCES AND COMPONENTS

RF Sources for High-Gradient Accelerator Research

MODIFIED MAGNICON FOR HIGH-GRADIENT ACCELERATOR R\&D

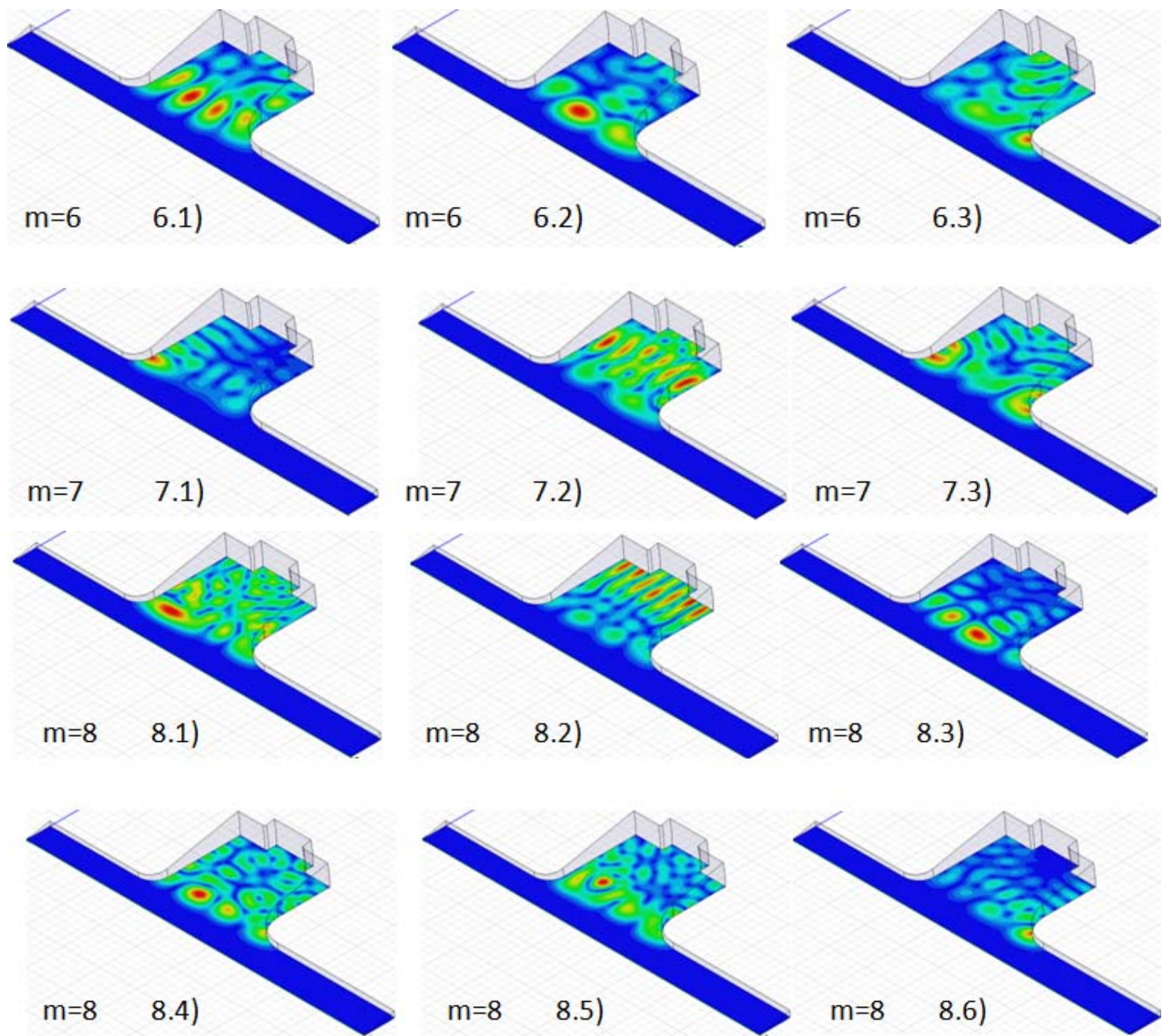

Fig. 34 (continued from previous page). HOMs as simulated by HFSS with $2 \leq \mathrm{m} \leq 9$

. For clarity we start the plot enumeration with 2 , corresponding to the azimuthal index $m$.

(continued on next page) 
Omega-P, Inc.

Phase II proposal submitted following Phase I DoE SBIR grant DE-SC000 4497

in response to DoE SBIR/STTR 2010 Solicitation

Topic 65c: RADIO FREQUENCY POWER SOURCES AND COMPONENTS

RF Sources for High-Gradient Accelerator Research

MODIFIED MAGNICON FOR HIGH-GRADIENT ACCELERATOR R\&D
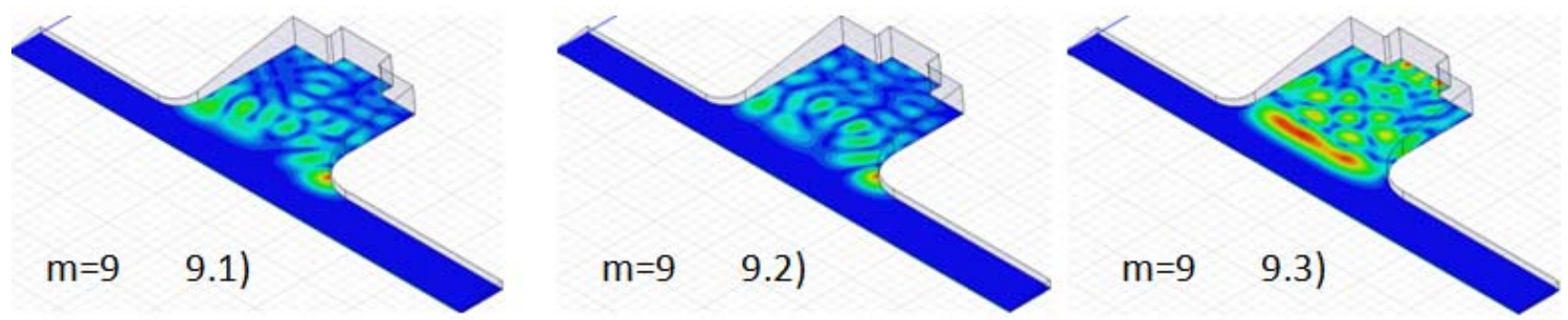

Fig. 34 (continued from previous page). HOMs as simulated by HFSS with $2 \leq \mathrm{m} \leq 9$

. For clarity we start the plot enumeration with 2 , corresponding to the azimuthal index $m$.

To find the geometry of the $6^{\text {th }}$ cavity where the HOMs shown in Fig. 34 are de-tuned sufficiently, a seed geometry was chosen with $\mathrm{R}=15.125 \mathrm{~mm}, \mathrm{~L}=12.2 \mathrm{~mm}, \mathrm{dR}=1.3366 \mathrm{~mm}$, $\mathrm{R} 1=2 \mathrm{~mm}, \mathrm{R} 4=4 \mathrm{~mm}$, and the rest of dimensions as in Table VII. The initial frequencies of the listed modes were found, along with the frequency derivatives $\partial / \partial \mathrm{R}, \partial / \partial \mathrm{L}, \partial / \partial(\mathrm{dR}), \partial / \partial \mathrm{R} 1$, and $\partial / \partial \mathrm{R} 4$. Five sizes were varied, namely the cavity radius $(\mathrm{R})$, length $(\mathrm{L})$, the size of the groove (dR), and R1 and R4 [see Fig 3.4) to achieve the de-tuning for all the HOMs near or above $\sim 200 \mathrm{MHz}$, while getting the working frequency to be 11,449 MHz. The results of this exercise suggest to have $\mathrm{R}=15.2 \mathrm{~mm}, \mathrm{~L}=12.275 \mathrm{~mm}, \mathrm{dR}=1.1656 \mathrm{~mm}, \mathrm{R} 1=2 \mathrm{~mm}$, and $\mathrm{R} 4=4$ $\mathrm{mm}$, which have been already listed in Table VII. The tolerances are obviously strict, and also have been listed in Table VII. The HOM frequencies and de-tunings are listed in Table VIII. The smallest de-tuning is $193 \mathrm{MHz}$ for the HOM shown in Fig 35; the de-tuning for $\mathrm{TM}_{322} / \mathrm{E}_{322}$ is $+246 \mathrm{MHz}$.

To summarize, the sizes of the version with the exit pipe radius $4.25 \mathrm{~mm}$ can be adjusted so that there is sufficient de-tuning for all HOMs (see Table VIII above) not to get excited. The cavity sizes (for the $6^{\text {th }}$ deflection cavity) have been presented already in Table VII.

\section{Modified $6^{\text {th }}$ cavity of the deflection system: summary}

It is desired to increase the exit drift tube radius of the $6^{\text {th }}$ deflection cavity to $4.25 \mathrm{~mm}$ to be able to use the deflection system at the drive power levels above 125 Watts. To remind, the present version of the deflection system has its $6^{\text {th }}$ cavity with the exit pipe radius $3.75 \mathrm{~mm}$. This present version, when operated at its optimum with the e-beam at $450 \mathrm{kV}$ produces a deflection angle that may force the e-beam to hit the pipe walls when the drive power exceeds 125-130 Watts. This imposes a limitation on the drive power level, and with it ultimately limits the efficiency of the magnicon. 
Omega-P, Inc.

Phase II proposal submitted following Phase I DoE SBIR grant DE-SC000 4497

in response to DoE SBIR/STTR 2010 Solicitation

Topic 65c: RADIO FREQUENCY POWER SOURCES AND COMPONENTS

$R F$ Sources for High-Gradient Accelerator Research

\section{MODIFIED MAGNICON FOR HIGH-GRADIENT ACCELERATOR R\&D}

Table VIII. The HOM frequencies $\boldsymbol{f}$ and their detuning $\mathrm{d} f$, in MHz.

\begin{tabular}{|c|c|c|c|c|}
\hline$m$ & $f(\mathrm{MHz})$ & $\mathrm{d} f(\mathrm{MHz})$ & $m \times 11,424(\mathrm{MHz})$ & Fig. 33. \\
\hline 2 & 24125 & 612 & 22848 & $2)$ \\
\hline 3 & 34928 & 246 & 34272 & $3)$ \\
\hline 4 & 44864 & -488 & 45696 & $4.1)$ \\
\hline 4 & 46884 & 534 & --- & $4.2)$ \\
\hline 5 & 56128 & -476 & 57120 & 5.1 \\
\hline 5 & 56321 & -534 & --- & 5.2 \\
\hline 5 & 58131 & 481 & --- & 5.3 \\
\hline 6 & 66769 & -997 & 68544 & 6.1 \\
\hline 6 & 69506 & 371 & --- & 6.2 \\
\hline 6 & 70794 & 1040 & --- & 6.3 \\
\hline 7 & 79346 & -413 & 79968 & 7.1 \\
\hline 7 & 81361 & 698 & --- & 7.3 \\
\hline 8 & 90569 & -437 & 91392 & 8.1 \\
\hline 8 & 90689 & -615 & --- & 8.2 \\
\hline 8 & 91281 & -209 & --- & 8.3 \\
\hline 8 & 91902 & 236 & --- & 8.4 \\
\hline 8 & 92237 & 341 & --- & 8.5 \\
\hline 8 & 93136 & 667 & --- & 8.6 \\
\hline 9 & 102608 & -193 & 102816 & 9.1 \\
\hline 9 & 104412 & 670 & -- & 9.2 \\
\hline 9 & 105463 & 1374 & --- & 9.3 \\
\hline
\end{tabular}

Fortunately, the $6^{\text {th }}$ cavity may be modified so that its exit pipe can be increased. The issues of having an impact on the e-beam dynamics, and/or of having a new spectrum of HOMs have been addressed and received a satisfactory solution. With the pipe radius increased to 4.25 $\mathrm{mm}$, it is possible now to operate at more than $125 \mathrm{~W}$ of drive power, gaining a good increment in device efficiency when operated at $200 \mathrm{~W}$ of the drive power. As a result, the produced power is boosted from $\sim 21 \mathrm{MW}$ to $\sim 27 \mathrm{MW}$. 


\section{Omega-P, Inc.}

Phase II proposal submitted following Phase I DoE SBIR grant DE-SC000 4497

in response to DoE SBIR/STTR 2010 Solicitation

Topic 65c: RADIO FREQUENCY POWER SOURCES AND COMPONENTS

$R F$ Sources for High-Gradient Accelerator Research

MODIFIED MAGNICON FOR HIGH-GRADIENT ACCELERATOR R\&D

\section{IId. Modifications to the magnetic system}

The Phase I work plan had among its tasks one dedicated to possible changes in the magnetic system to boost the performances of the newly proposed tube. More specifically Task 3 was formulated as follows: “...the possibility of an iron ring-like screen (e.g. made out of permendure) between the deflection cavity chain and the output cavity to prevent the magnetic flux exchange, and thus, guarantee the independence of electron beam dynamics in the deflection cavity chain from the magnetic fields created by the coils around the output cavity and vice versa will be investigated. That should insure more flexibility in optimization. The steps of Tasks \#1 and 2 will be repeated, and a decision made whether the [ring-like] screen is worth implementing..." In this sub-section we are addressing this topic.

The deflection cavity chain followed by the output cavity is shown in Fig. 35 (left-hand side). There is enough room (see the right-hand side) to insert a permeable ring between the last deflection cavity (\#6) and the output cavity. A possible way to position such a ring accurately is to somewhat re-design the brazed joints and have a groove-like opening in the copper body for the ring to be inserted. However, before going to that extent, benefits of having the ring must be investigated. The function of the ring is to separate the magnetic fluxes upstream of it and downstream of it, and thus to be able to fine-tune the profiles of the guiding magnetic field in the deflection system and the output cavity independently. The downstream flux is presently created by the coil triplet, which is situated around the output cavity and collector, and one bucking coil. Having the ring separating this flux from other contributors can be advocated, if the current in the aforementioned coils can be fine-tuned to boost the efficiency of interaction between the ebeam and the RF-fields in the output cavity. However, it was shown in Phase I Proposal [see p. 12, Fig. 10] that the efficiency of interaction is largely insensitive to variations in the current of the bucking coil as well as to variations in the current of the triplet formed by the output coils [wired in series]. In this sub-section, we shall present this argument again. The simulations to prove the fact cited above were performed in the configuration which has the last deflection cavity (\#6) as per the design presented already in Sub-Section IIc, and the output cavity as per the design presented already in Sub-Section IIb. No ring was introduced so far.

Fig. 36 demonstrates that, indeed, the current in the coils [of interest] may vary a lot, yet the efficiency still remains reasonably high. This, of course, should be expected because the overall idea here is to build a device which is robust, and in particular is not much sensitive to the magnetic field magnitude and profile in the output cavity. Thus, having a ring to separate

the downstream flux from other contributors is not necessary because the efficiency of interaction is largely insensitive to the magnitude and the details of the profile of guiding magnetic field in the output cavity. 
Omega-P, Inc.

Phase II proposal submitted following Phase I DoE SBIR grant DE-SC000 4497

in response to DoE SBIR/STTR 2010 Solicitation

Topic 65c: RADIO FREQUENCY POWER SOURCES AND COMPONENTS

$R F$ Sources for High-Gradient Accelerator Research

MODIFIED MAGNICON FOR HIGH-GRADIENT ACCELERATOR R\&D

The presence of a ring might still be advocated, if it can be shown that the magnetic flux upstream of it (in the deflection cavity chain) might be fine-tuned to force the deflection system to provide larger deflection angles (after the sixth cavity) as compared to the situation when no ring is present. Experience with the former magnicons suggests that typically nearly best performances are achieved when the field profile across the deflection cavities is nearly uniform. It is also typical then that the amount of drive power required to have large deflection angles at the end of the deflection system is reduced.

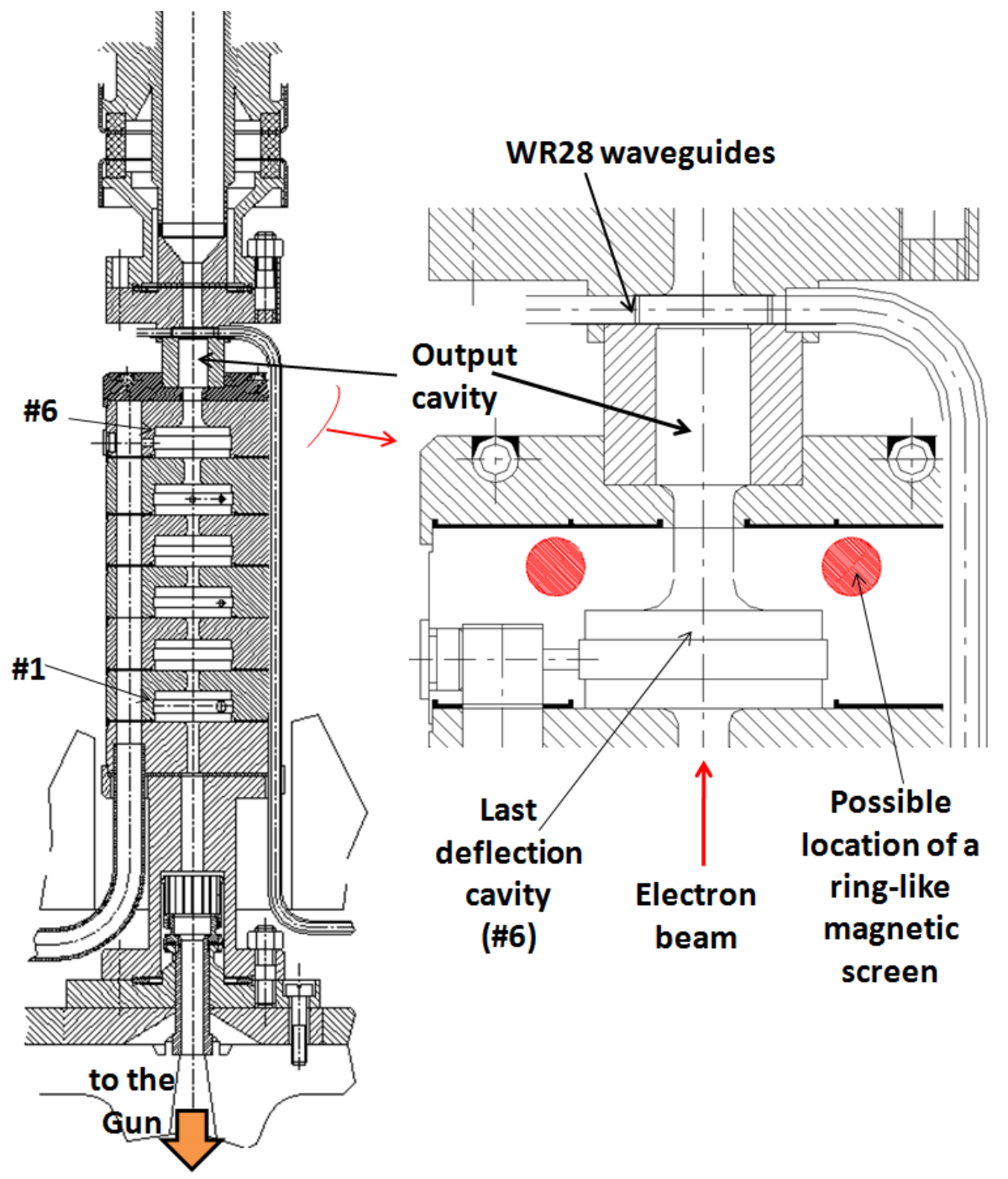

Fig. 35. (left) Deflection cavity chain (\#1 - \#6) followed by the output cavity and collector [which will be modified as described in Sub-Section IIe], and (right) the possible place to insert a ring-like magnetic screen. 


\section{Omega-P, Inc.}

Phase II proposal submitted following Phase I DoE SBIR grant DE-SC000 4497

in response to DoE SBIR/STTR 2010 Solicitation

Topic 65c: RADIO FREQUENCY POWER SOURCES AND COMPONENTS

RF Sources for High-Gradient Accelerator Research

MODIFIED MAGNICON FOR HIGH-GRADIENT ACCELERATOR R\&D

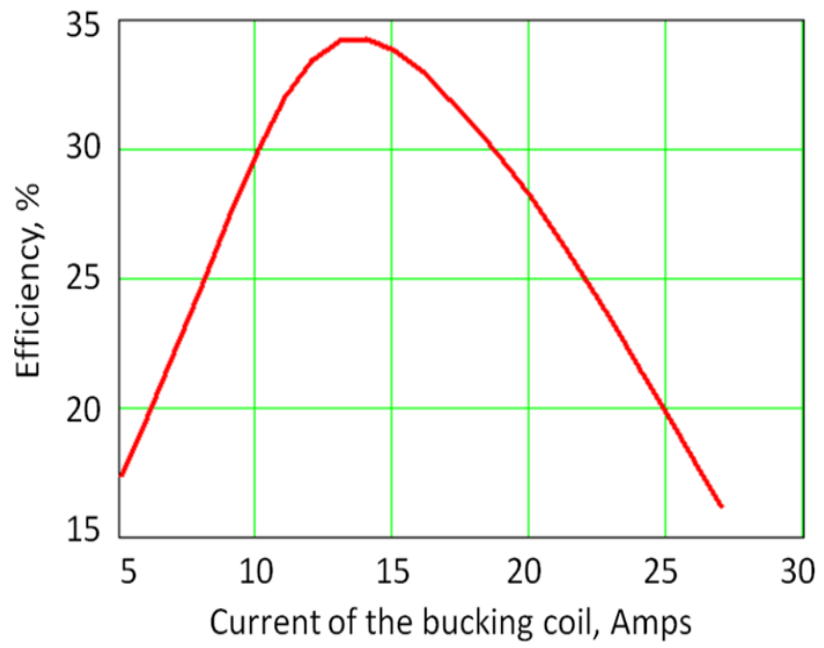

a)

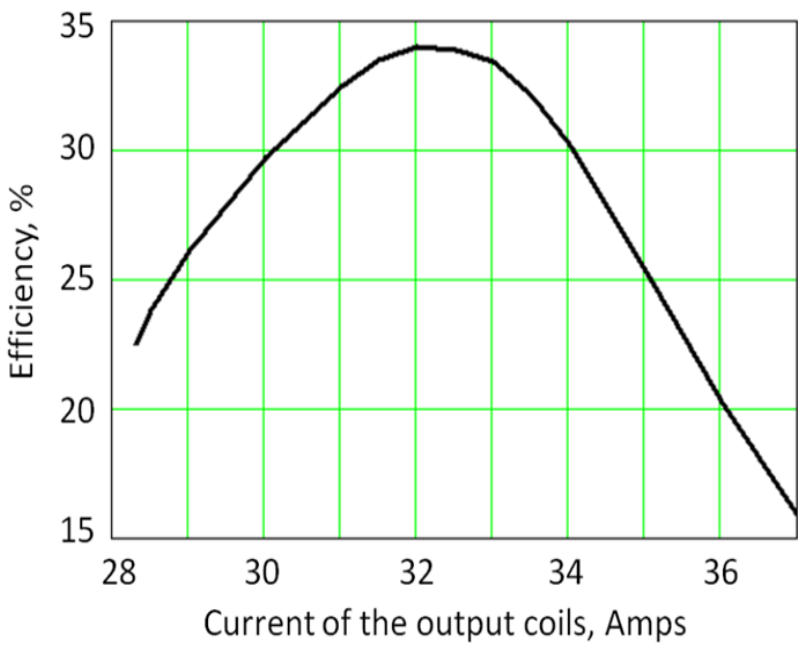

b)

Fig. 36. (a) Efficiency $v s$ the current of the bucking coil, and (b) efficiency $v s$ the current of the output coils wired together in series (totally there are 3 coils). One observes that the bucking coil' current may vary from 8 to $22 \mathrm{~A}$, but the efficiency still will be above $25 \%$.

Also, one sees that the current of the output coils may vary from 29 to $35 \mathrm{~A}$ and the efficiency still be above $25 \%$. (The current set-points in this simulation were: $86 \mathrm{~A}$ in all the coils around the deflection cavities [in actuality, these coils are wired in series], $12 \mathrm{~A}$ in the bucking coil, and $33 \mathrm{~A}$ in all the output coils [in actuality, these coils are wired in series].)

Indeed, Fig. 37a shows that with a nearly uniform field profile, which might be achieved if the ring is placed between the last deflection cavity (\#6) and the output cavity to separate the fluxes upstream and downstream of it, optimization may be performed to have the deflection angle 38-39 deg with a drive power of $\sim 70 \mathrm{~W}$. However, one can achieve even slightly better performance [see Fig. 37b] when the magnetic field profile is not uniform (as provided by the magnetic system as already is). An angle of $40.5 \mathrm{deg}$ is possible, with only the penalty that the drive power must be $200 \mathrm{~W}$. This drive power is readily available. Given these facts, one concluses that the deflection system performs well without the ring-like screen.

[For a reader unfamiliar with the typical behavior of the magnicon deflection system, it can be useful to note that the deflection angle grows when the drive power is increased; this growth continues to a certain level of the drive power. (In the aforementioned examples this level is 70 and 200 Watts respectively.) Then the deflection system saturates and the deflection angle at its exit does not get larger even if the drive power is increased; this is valid up to a certain threshold level of power, after which the deflection system falls into a nonlinear regime and the performance typically deteriorate severely.] 


\section{Omega-P, Inc.}

Phase II proposal submitted following Phase I DoE SBIR grant DE-SC000 4497

in response to DoE SBIR/STTR 2010 Solicitation

Topic 65c: RADIO FREQUENCY POWER SOURCES AND COMPONENTS

$R F$ Sources for High-Gradient Accelerator Research

MODIFIED MAGNICON FOR HIGH-GRADIENT ACCELERATOR R\&D

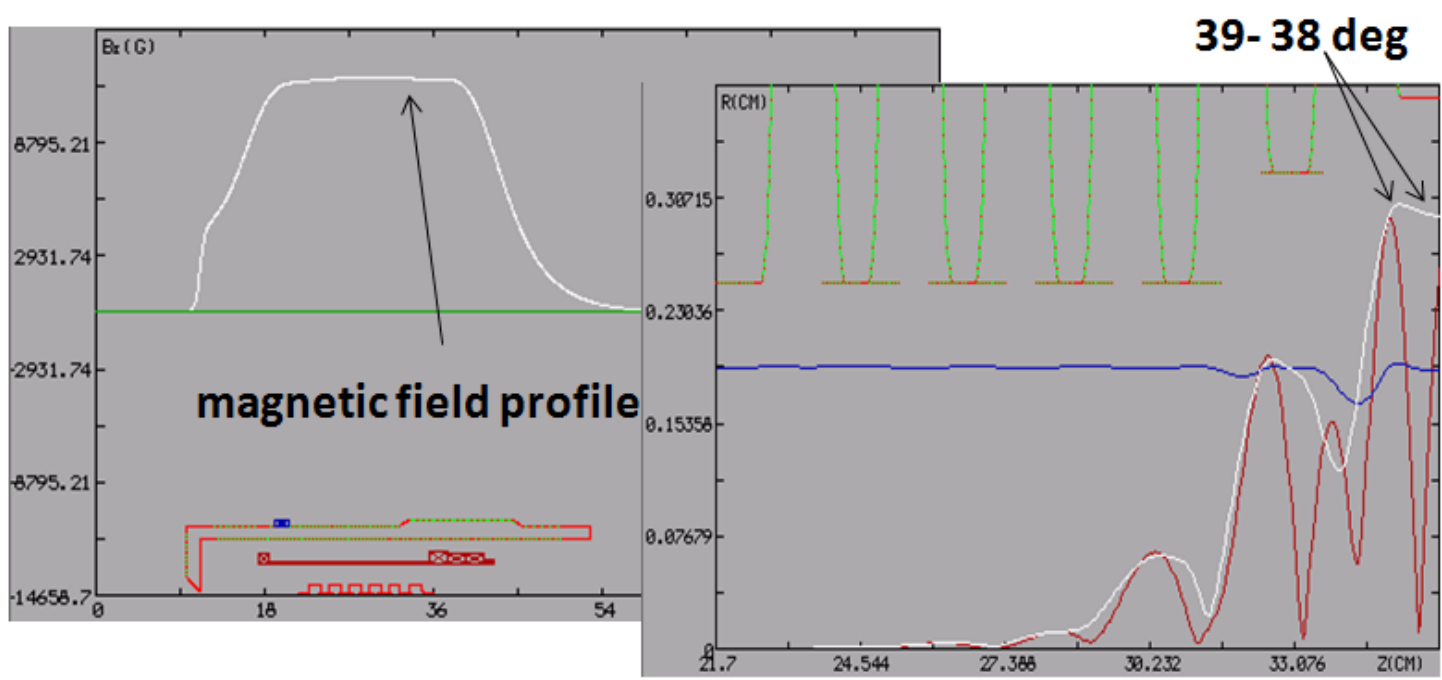

a)

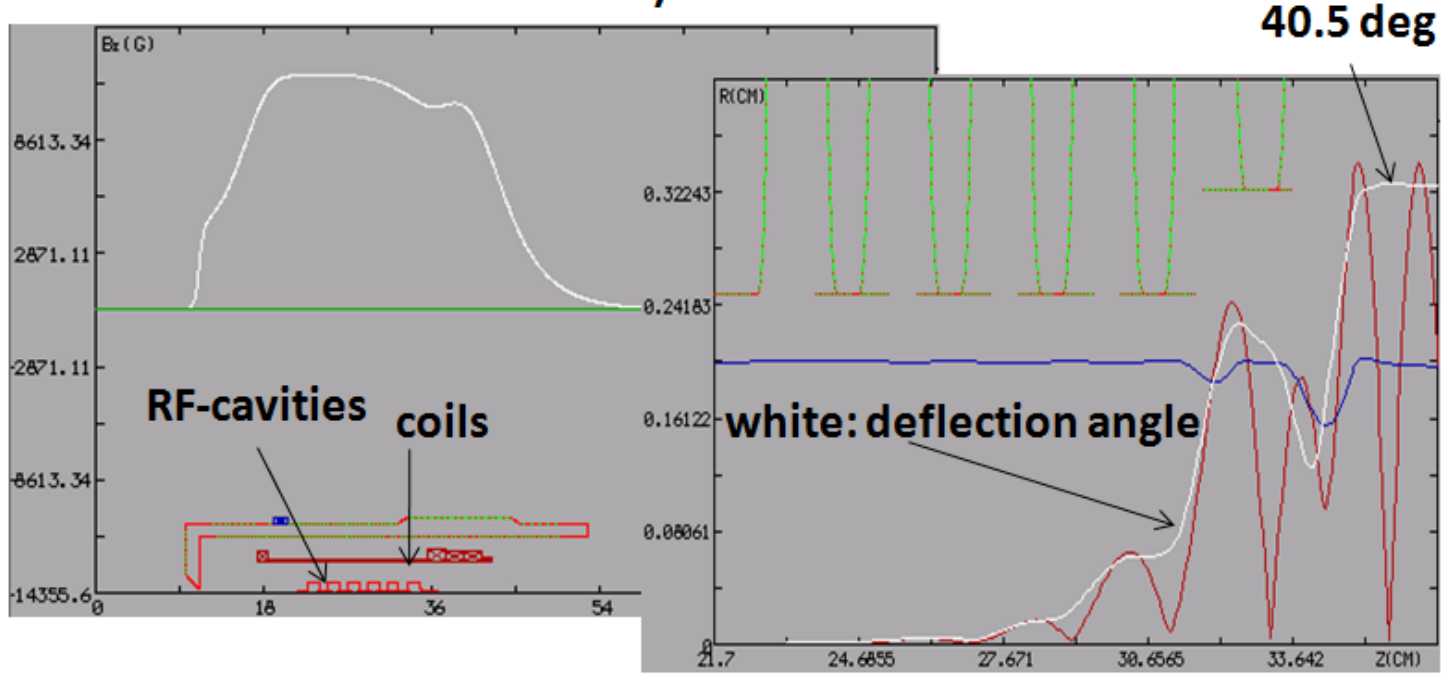

b)

Fig. 37. (a) With a uniform magnetic field profile (which could be achieved if a ring-like screen is placed after the last deflection cavity, but before the output cavity, to separate the flux upstream of the ring from the flux downstream of the ring), the deflection system can be optimized to have at its exit an average deflection angle of 38-39 deg at a drive power level of $70 \mathrm{~W}$; (b) However, with a non-uniform profile (which happens with the magnetic system as already is), the deflection system can be optimized even somewhat better and gives at its exit a deflection angle of $40.5 \mathrm{deg}$ [in average]. The penalty of having this angle is need for $200 \mathrm{~W}$ of drive power; however, this drive power is readily available, and the gain reduction of about 4.5 $\mathrm{dB}$ is inconsequential for the Test Facility application for which the tube is intended. 


\section{Omega-P, Inc.}

Phase II proposal submitted following Phase I DoE SBIR grant DE-SC000 4497 in response to DoE SBIR/STTR 2010 Solicitation Topic 65c: RADIO FREQUENCY POWER SOURCES AND COMPONENTS $R F$ Sources for High-Gradient Accelerator Research MODIFIED MAGNICON FOR HIGH-GRADIENT ACCELERATOR R\&D

To summarize, it has been found that, with the present magnetic coil configuration and already installed pole-pieces, the guiding magnetic field can be optimized so well that the deflection system provides large angles which are suitable to achieve an efficiency above $30 \%$. The output cavity generates power efficiently within a broad range of the magnetic field magnitude values; power generation is not sensitive much to details of the guiding magnetic field in the output cavity. Consequently, no additional rings, screens or pole-pieces need be used or necessary at all to boost the performance of the device; and thus, in the new modified magnicon, the magnetic system will be used in the very same configuration as is already.

\section{IIe. Modifications to the collector}

In the Phase I proposal, Task 6 states that "... a new collector will be designed, without ceramic insertions to have capability to braze it to the output cavity..." It was further stipulated that "...work is to be applied ... so as to effect its smooth connection to the cavity chain ..., but otherwise without major changes from its present design..." It was also noted that "...a new design will repeat (most likely) the design of the present unit; however, the conflat flange at the entrance will be discarded..." Because there will be no ceramic ring between the output cavity and collector, the electron beam current value will be determined on the basis of the knowledge of the gun perveance, that is well known, and has been proven not to change since the gun (which is being presently used) was already well conditioned. The collector brazed to the output cavity, that is, in turn, brazed to the deflection cavity-chain will result in having a hightemperature bake-able structure - not available with the present tube.

As shown in Fig 38a, the collector is presently connected to the output cavity via a conflate flange (pos.1), and there is a ceramic insert (pos. 2) that serves to insulate the main body of collector from the cavity chain (so that a resistor may be connected to main collector body to drain charges and thus measure the voltage [shape] to infer later the current profile). However, this ceramic insert limits the possibility to bake only to very modest temperatures $\left(150-200{ }^{0} \mathrm{C}\right)$. Moreover, the pocket in the structure near the ceramic is a space in which a spurious mode might dwell. One may simply discard both the conflate flange and the ceramic insert [see Fig.5.1.b], but otherwise retain the inner configuration of the collector so that there is no chance for spurious modes existing between collector and the output cavity to appear because they were suppressed in the earlier version. The insert in pos. 3 also goes away; a brazing or welding joint (pos.4) will be placed where the conflate flange was.

A possibility to split the collector body into three segments by a 304 stainless steel insert

in pos. 5 has been considered. The insert might be welded. This still should allow one to fully bake the structure at high temperatures. The insert might serve as a resistor via which the 


\section{Omega-P, Inc.}

Phase II proposal submitted following Phase I DoE SBIR grant DE-SC000 4497 in response to DoE SBIR/STTR 2010 Solicitation

Topic 65c: RADIO FREQUENCY POWER SOURCES AND COMPONENTS $R F$ Sources for High-Gradient Accelerator Research MODIFIED MAGNICON FOR HIGH-GRADIENT ACCELERATOR R\&D

charges are drained, allowing some voltage to develop, which one might measure to infer the current profile. However, given the sizes of the insert its total resistance amounts only to about $R=6 \times 10^{-7}$ Ohms. On the other hand the capacitance of the collector main body amounts to about $20 \mathrm{pF}$. All together, this means that the voltage drop is determined by the resistance of the insert, and this is $V_{\text {drop }}=R \times I=\sim 0.1 \mathrm{mV}$ for the expected e-beam current of $187 \mathrm{~A}$. The value is too small to be measured given the amount of parasitic noise permeating the laboratory environment when the tube is pulsed. Consequently, it is not planned to use any splitting steel inserts in the collector main body unless other merits may be found. In summary, the design as shown in Fig. 38b, is a working design for the moment.

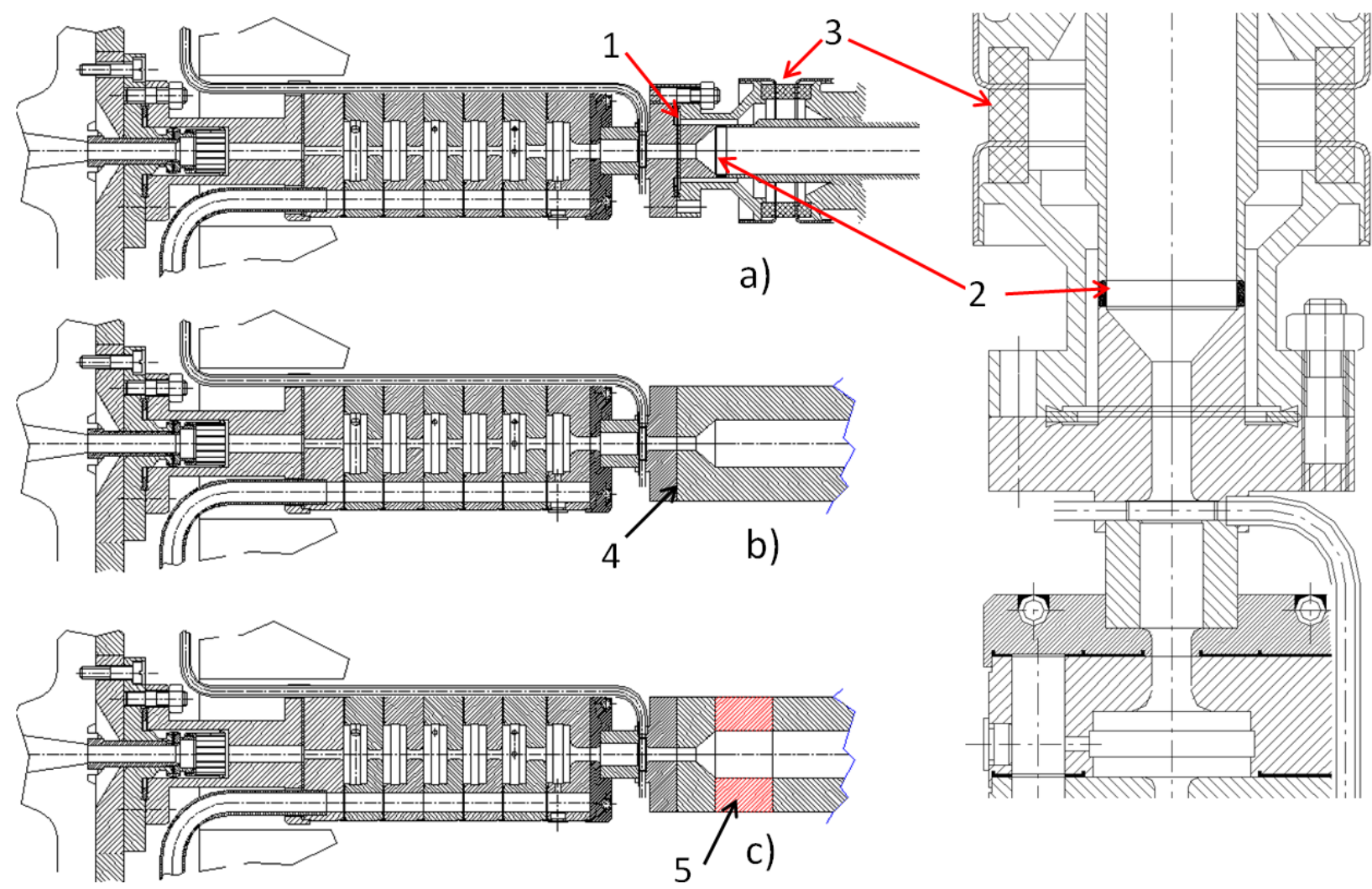

Fig. 38. Possible collector configurations. [See text for explanations] 
Omega-P, Inc.

Phase II proposal submitted following Phase I DoE SBIR grant DE-SC000 4497

in response to DoE SBIR/STTR 2010 Solicitation

Topic 65c: RADIO FREQUENCY POWER SOURCES AND COMPONENTS

$R F$ Sources for High-Gradient Accelerator Research

MODIFIED MAGNICON FOR HIGH-GRADIENT ACCELERATOR R\&D

\section{IIf. Decision making process when designing and optimizing the output cavity.}

In this sub-section, the decision-making process to design and optimize the output cavity is presented in details in its actual sequence.

\section{Choosing the Q-factor of output cavity}

Studying the beam dynamics [see Fig 39], one concludes that having a lower $Q$-factor delivers a lower field strength in the output cavity, while delivering almost the same efficiency. An example for a simple pillbox cavity (with length $2.15 \mathrm{~cm}$ ) that has two axial drift tubes (with radius of $4.75 \mathrm{~mm}$ ) to let the e-beam in and out is shown in Fig. 40. It should be noted that the same generic behavior is observed for more complex cavity geometries, even though the numbers for efficiency and the field strength are somewhat different. Practically for this cavity operating at $\mathrm{H}_{311} / \mathrm{TE}_{311}$-mode, it is, however, very challenging to design a coupling scheme that provides low $Q$-factors. A practical choice is to strive to achieve a $Q$-factor around 200. Several schemes were considered, and are reviewed below.

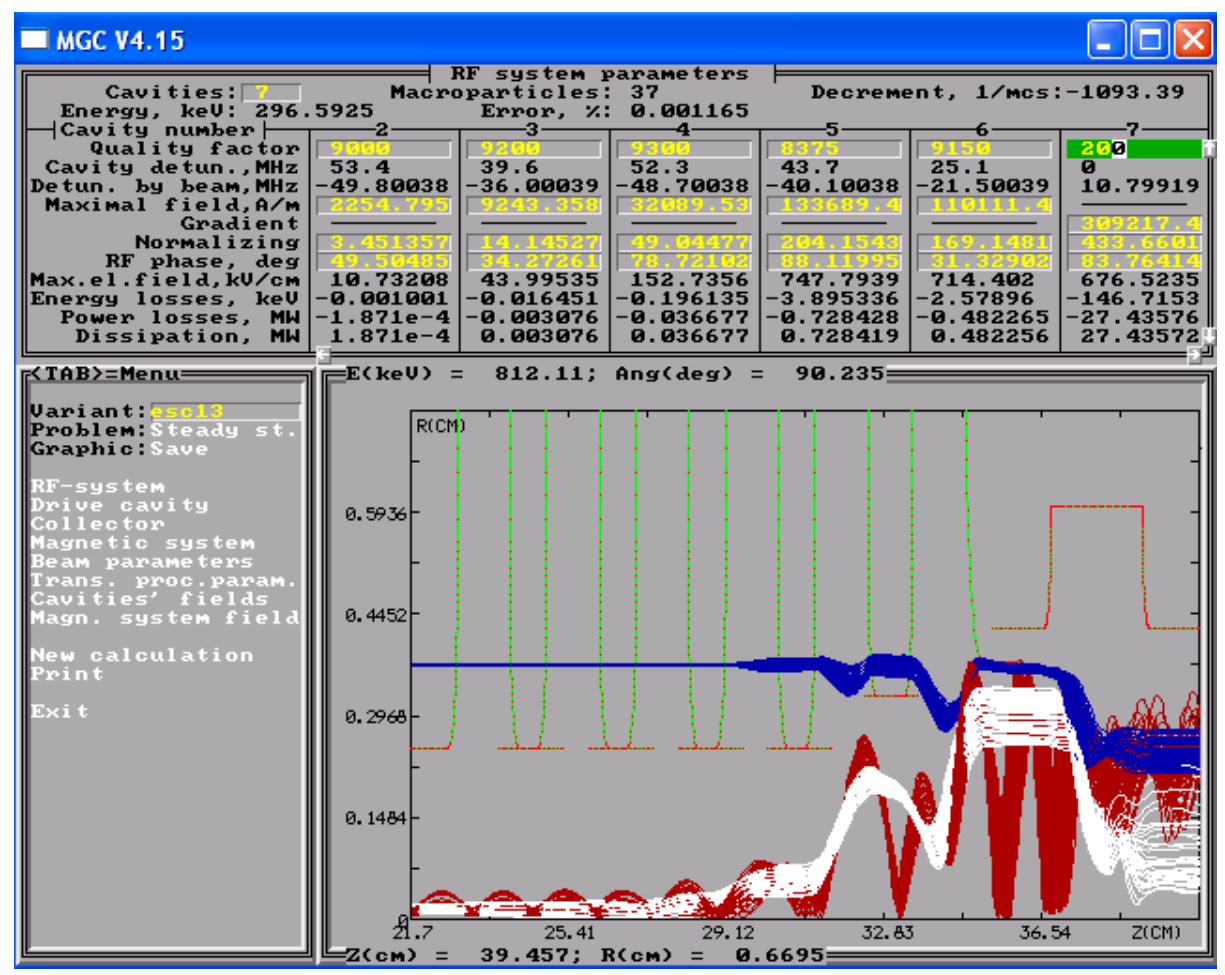

Fig. 39. Beam dynamics in the magnicon. Blue curves are particle energies, red curves are magnitudes of particle radii, and white curves are deflection angles. 
Omega-P, Inc.

Phase II proposal submitted following Phase I DoE SBIR grant DE-SC000 4497

in response to DoE SBIR/STTR 2010 Solicitation

Topic 65c: RADIO FREQUENCY POWER SOURCES AND COMPONENTS

$R F$ Sources for High-Gradient Accelerator Research

MODIFIED MAGNICON FOR HIGH-GRADIENT ACCELERATOR R\&D
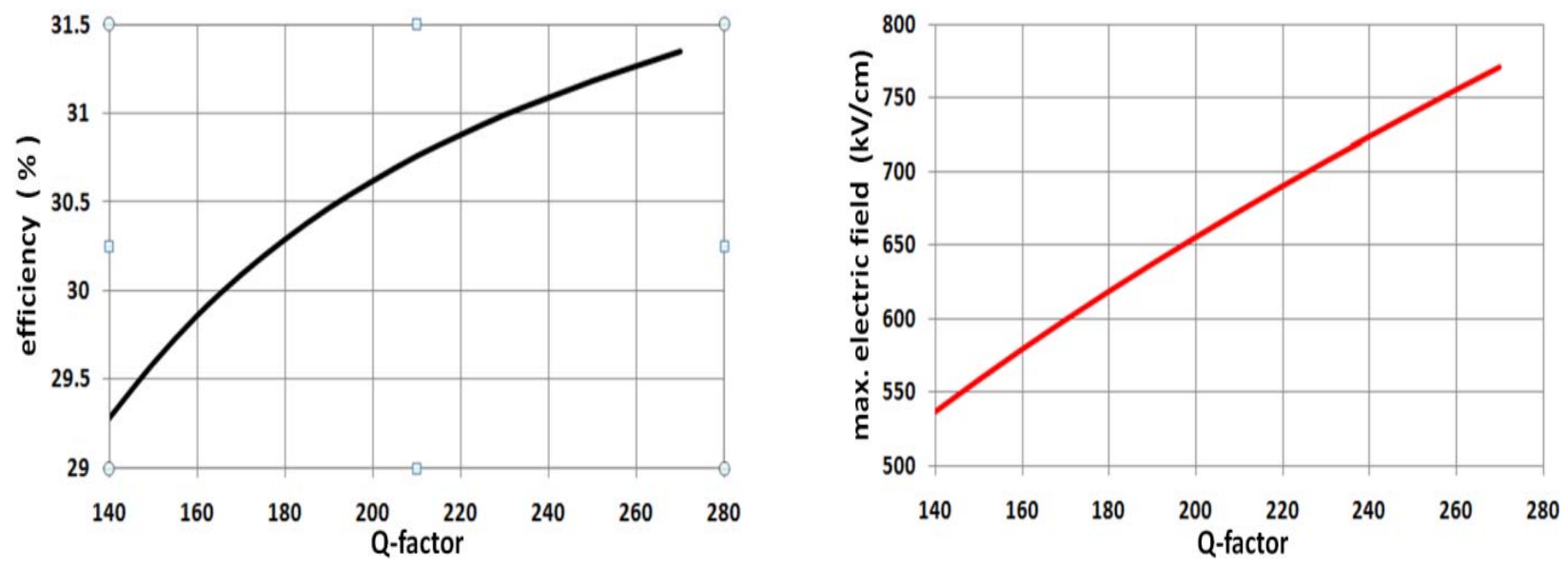

Fig. 40. Efficiency and maximum surface electric field $v s$ output cavity $Q$-factor. Note that the $Q$-factor is the total $Q$-factor arising from both the finite wall conductivity (a minor contribution) and the external loading (the major contribution).

\section{Coupling schemes to obtain the required Q-factor for the output cavity}

Since the new cavity is to be integrated into the old RF power distribution layout in the lab where user's experiments are situated, it is required to have four ports for each of already present four waveguide transmission lines. One of the schemes considered was to have four coupling slots in the middle of the cavity [see Fig. 41] with different waveguide position as viewed from the back. However, to achieve a low $Q$-factor ( 200), the slot dimensions (length) must be of that of a near resonance slot; given this, the electromagnetic field primarily concentrates in the slots themselves [see Fig. 42], which reduces the effective interaction length to no more than $1.5 \mathrm{~cm}$, and thus requires a high field strength (typically in excess of $770 \mathrm{kV} / \mathrm{cm}$ ) to maintain the desired efficiency ( $30 \%$ or more). This approach has thus been discarded.

An alternative, and somewhat better, design exits. The cavity comprises two chambers: the $1^{\text {st }}$ chamber has the field pattern to effectively interact with the e-beam, and the $2^{\text {nd }}$ chamber has a field pattern to properly load the entire cavity. This design has been proposed earlier in Phase I, and is described here. Two examples of a composite cavity are shown in Fig. 43; they differ by having different shapes for the $1^{\text {st }}$ chamber [that is, having different $R_{\text {wall }}(z)$-profiles, with $\mathrm{z}$ being the usual coordinate along the cavity axis, and $R_{\text {wall }}$ being the radial coordinate of the wall surface]. To remind, in the Phase I proposal, other possibilities were considered, but those presented in Fig. 43 were found to be more promising to provide field patterns that have low electric fields on the metal surface while still having a relatively large efficiency. 


\section{Omega-P, Inc.}

Phase II proposal submitted following Phase I DoE SBIR grant DE-SC000 4497

in response to DoE SBIR/STTR 2010 Solicitation

Topic 65c: RADIO FREQUENCY POWER SOURCES AND COMPONENTS

RF Sources for High-Gradient Accelerator Research

\section{MODIFIED MAGNICON FOR HIGH-GRADIENT ACCELERATOR R\&D}
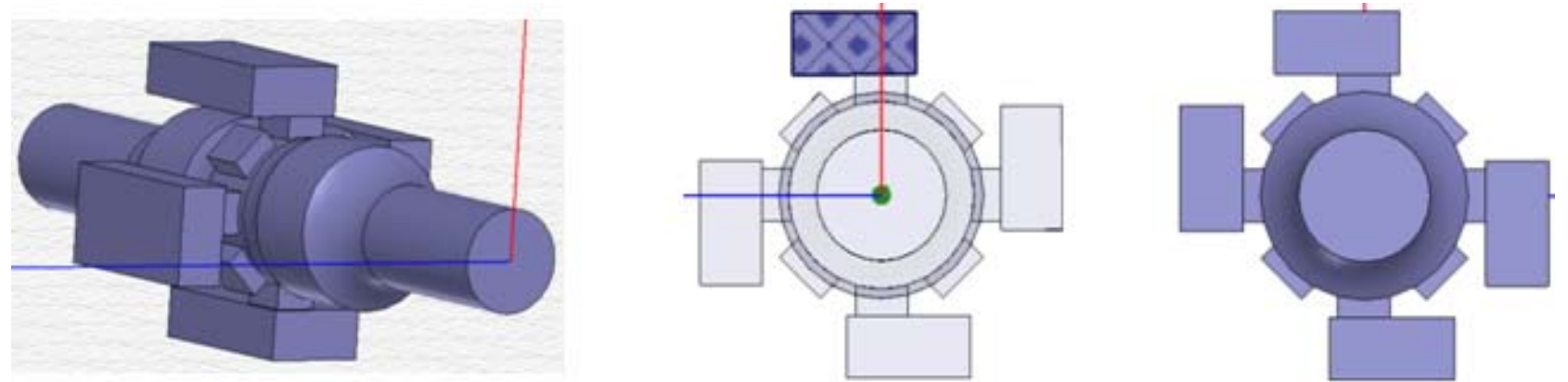

Fig. 41. Output waveguide coupling. Two pictures at the right are rear view, and show possible waveguide position relative to the coupling slots.
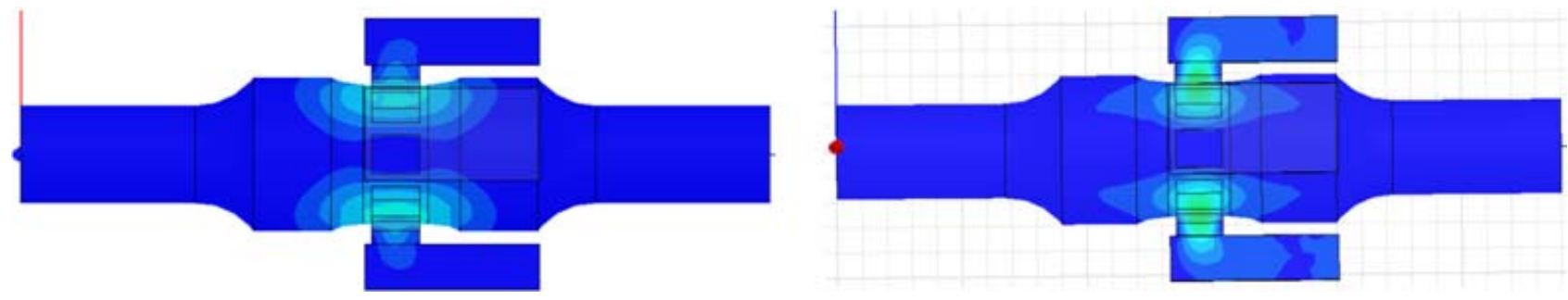

Fig. 42. HFSS simulations show the patterns of total complex magnitude of the electric field in the $z-x$ and $z-y$ planes (on the right), with $z$ being the axial coordinate of the cavity. The patterns look different because the presence of only four coupling slots breaks the azimuthal, sextuple symmetry of the operational $\mathrm{TE}_{311} / \mathrm{H}_{311}$-mode. Additional protrusions can be made [see Fig.6.3] but given the available spacing, these are not nearly enough to compensate the distortions.

Observe that the patterns show that the field concentrates near the slots. 
Omega-P, Inc.

Phase II proposal submitted following Phase I DoE SBIR grant DE-SC000 4497

in response to DoE SBIR/STTR 2010 Solicitation

Topic 65c: RADIO FREQUENCY POWER SOURCES AND COMPONENTS

$R F$ Sources for High-Gradient Accelerator Research

MODIFIED MAGNICON FOR HIGH-GRADIENT ACCELERATOR R\&D
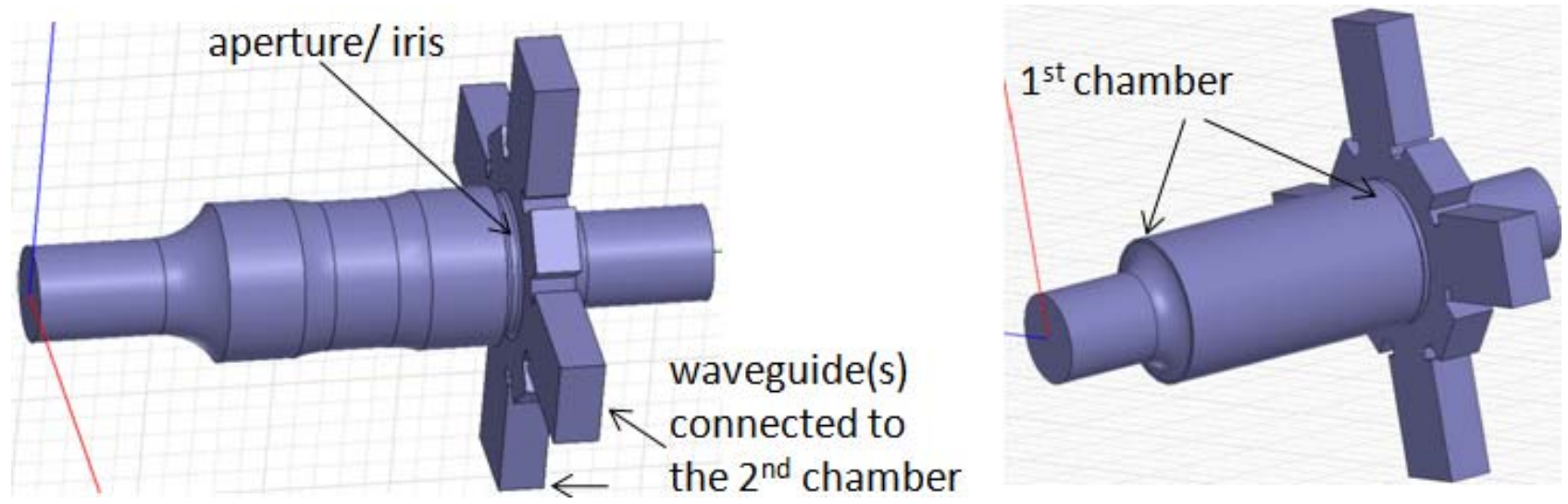

Fig. 43. Configuration of the composite cavity that has two chambers. The first one sustains the interaction between the e-beam and the electromagnetic fields, while the second one connects to the four waveguides to obtain the required loaded $Q$-factor $(\sim 200)$. Note that the shape of the $1^{\text {st }}$ chamber may vary to provide best field patterns to have low electric fields on the surface while having a still large efficiency - compare the pictures on the left (profiled chamber) and right (smooth-bore chamber).

For a simplified characterization, the $1^{\text {st }}$ chamber operates in that $\mathrm{TE}_{311} / \mathrm{H}_{311}$-mode as needed, while the $2^{\text {nd }}$ operates in the $\mathrm{TM}_{310} / \mathrm{E}_{310}$-mode, where both modes are tightly coupled via the aperture formed by an iris between two cavities. The aperture formed by the iris - even being a relatively shallow feature as compared to the $1^{\text {st }}$ cavity diameter - is essential to maintain the field distribution in the $1^{\text {st }}$ chamber. It is possible to find a geometry of the $2^{\text {nd }}$ chamber so that with four waveguides the field patterns in the $1^{\text {st }}$ chamber are those of sextuple type even if in the $2^{\text {nd }}$ chamber itself field distortions are substantial [see Fig. 44].
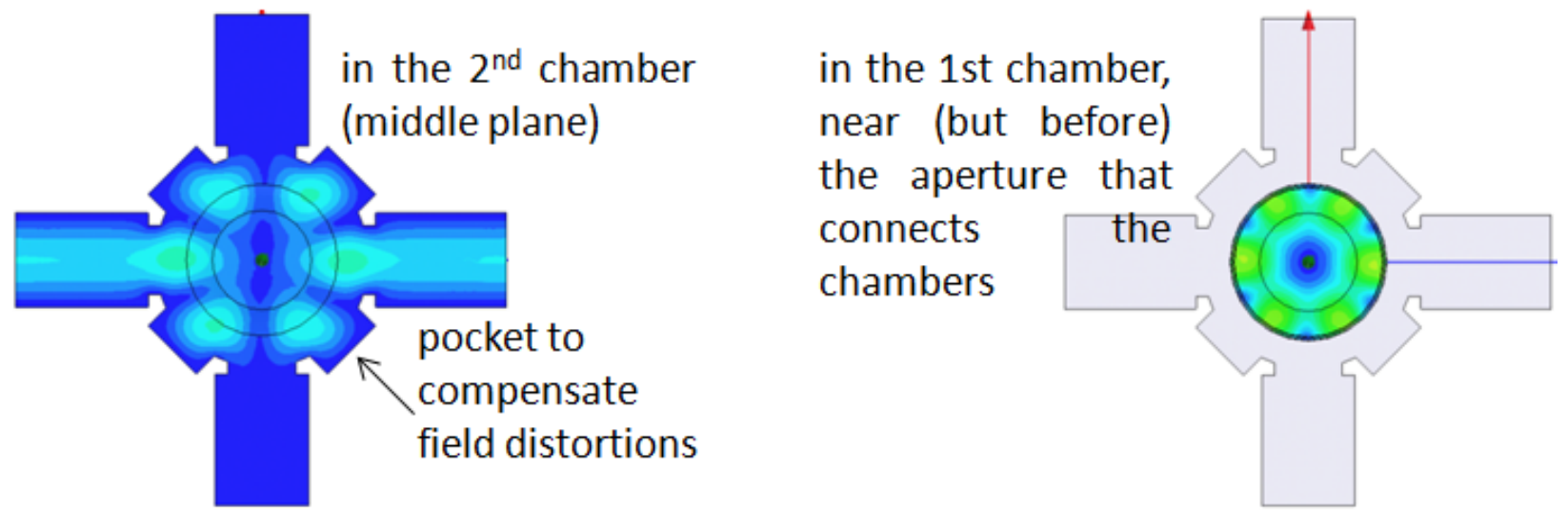

Fig. 44. Field maps in the two chambers of the output cavity. (See text for explanations.) 
Omega-P, Inc.

Phase II proposal submitted following Phase I DoE SBIR grant DE-SC000 4497

in response to DoE SBIR/STTR 2010 Solicitation

Topic 65c: RADIO FREQUENCY POWER SOURCES AND COMPONENTS

RF Sources for High-Gradient Accelerator Research

MODIFIED MAGNICON FOR HIGH-GRADIENT ACCELERATOR R\&D

\section{Cavity longitudinal shape to maximize efficiency}

To answer the question as to the longitudinal shape of the $1^{\text {st }}$ chamber taking into account the practically achievable $Q$-factor of $\sim 200$ (as it has been explained above), the length of the $1^{\text {st }}$ chamber must exceed $2.2 \mathrm{~cm}$ to have low field magnitudes (as it was demonstrated in the Phase I proposal), different shapes were checked again. The simulations were done with two wellknown program packages: CLANS2 (2003 version) and MGC v.4.15 supported for compatibility with each other by Axif9/Decod- family programs. Results of particle dynamics simulations for different longitudinal shapes of the $1^{\text {st }}$ chamber are presented graphically in Fig. 45, and summarized below in Table IX.

Table IX.

\begin{tabular}{|c|c|c|}
\hline shape narrative descriptor & max. electric field $(\mathrm{kV} / \mathrm{cm})$ & efficiency $(\%)$ \\
\hline smooth-bore & 655 & 30.6 \\
\hline "butterfly" & 892 & 31.85 \\
\hline "spindle" & 936 & 32.3 \\
\hline "inverse spindle" & 918 & 31.06 \\
\hline
\end{tabular}

One observes that given all the requirements, the smooth-bore configuration (which is essentially a simple pillbox cavity) involves a substantially lower electric field strength (measured on the wall surface) while having almost the same efficiency as the other versions. Thus, the smoothbore design becomes the design of choice. The data presented also suggest that the efficiency of interaction between the e-beam and the fields in the output cavity is very insensitive to the cavity shape profile in the longitudinal direction (that is, the interaction is insensitive to any minute details in the longitudinal field distribution which is dictated, of course, by the cavity shape); however, in most cases the field magnitudes will grow substantially if a shape, which is different from that of a simple pillbox cavity, is selected. With the longitudinal shape of the $1^{\text {st }}$ chamber selected, the chamber length becomes the next question. 


\section{Omega-P, Inc.}

Phase II proposal submitted following Phase I DoE SBIR grant DE-SC000 4497 in response to DoE SBIR/STTR 2010 Solicitation

Topic 65c: RADIO FREQUENCY POWER SOURCES AND COMPONENTS

$R F$ Sources for High-Gradient Accelerator Research

\section{MODIFIED MAGNICON FOR HIGH-GRADIENT ACCELERATOR R\&D}

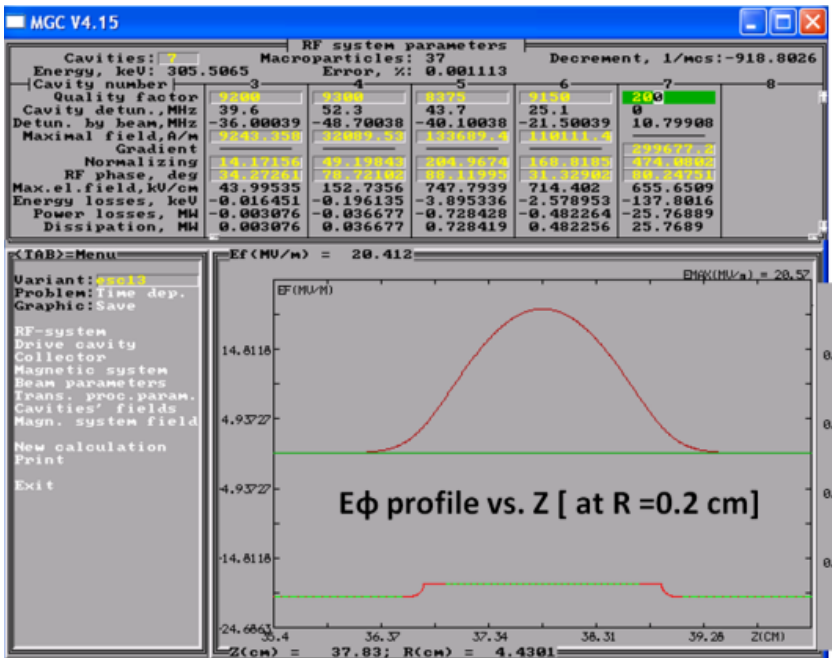

Smooth-bore version

Max. electric field $655 \mathrm{kV} / \mathrm{cm}$

Efficiency $30.6 \%$
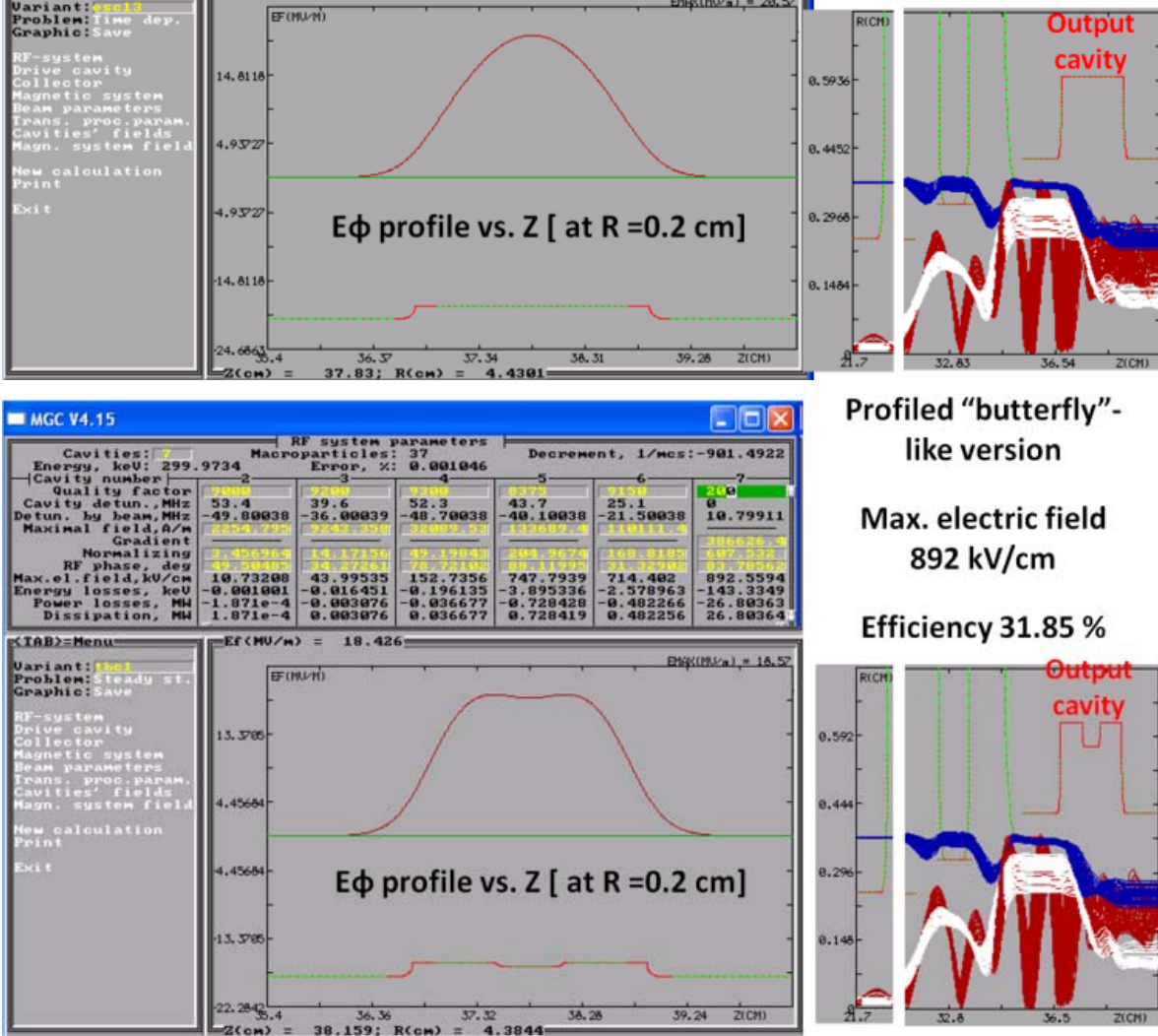

Profiled "butterfly"like version

Max. electric field 892 kV/cm

Efficiency $31.85 \%$

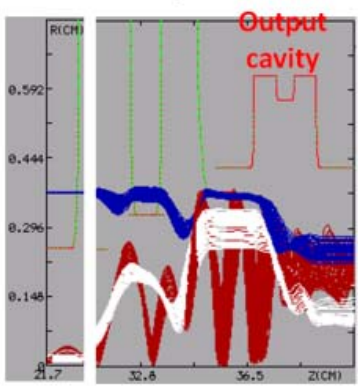

Fig. 45 (continued on the next plot) 


\section{Omega-P, Inc.}

Phase II proposal submitted following Phase I DoE SBIR grant DE-SC000 4497

in response to DoE SBIR/STTR 2010 Solicitation

Topic 65c: RADIO FREQUENCY POWER SOURCES AND COMPONENTS

$R F$ Sources for High-Gradient Accelerator Research

\section{MODIFIED MAGNICON FOR HIGH-GRADIENT ACCELERATOR R\&D}

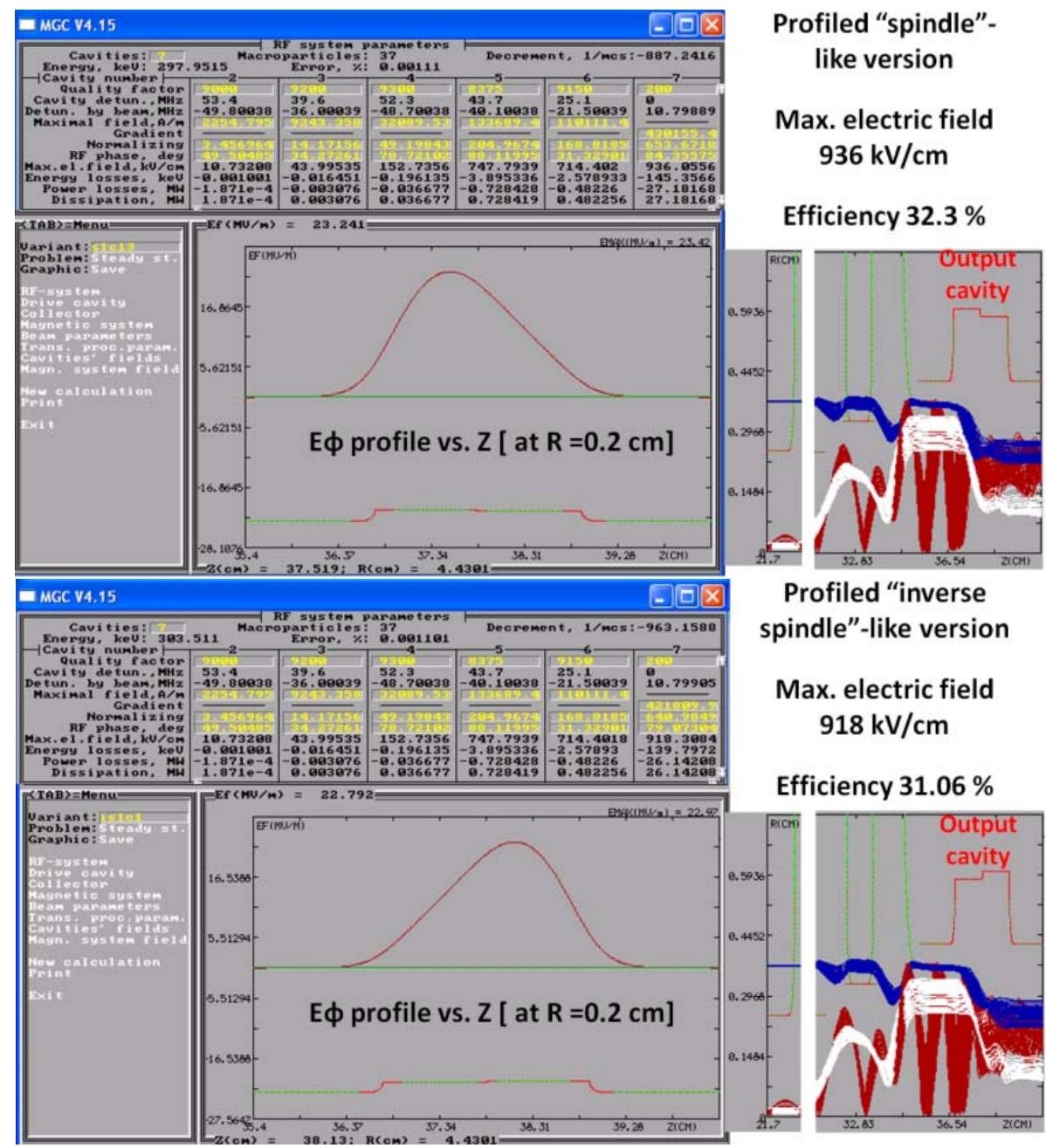

Fig. 45 (continued). Results of dynamics simulations for different longitudinal shapes of the $1^{\text {st }}$ chamber (with length $2.15 \mathrm{~cm}$, preceded and followed by drift tubes with radius $0.425 \mathrm{~cm}$ ), which provide different field profiles across the cavity - see e.g. the $E \varphi v z z$ (at $r=0.2 \mathrm{~cm}$ ) on every plot. On the left-hand side of each plot the behavior of the e-beam in the cavity is shown as a collection of curves: each of blue curves gives the evolution of energy of an individual macroparticle; red curves give the evolution of the macro-particle's radii as the e-beam progress down the cavity; and each of white curves shows the angle between the cavity axis and a given macroparticle. (Note that on the left-hand side subplot, the scales for $r$ and $z$ are different for the sake of presentation.) The total $Q$-factor is 200 in all cases. 


\section{Omega-P, Inc.}

Phase II proposal submitted following Phase I DoE SBIR grant DE-SC000 4497

in response to DoE SBIR/STTR 2010 Solicitation

Topic 65c: RADIO FREQUENCY POWER SOURCES AND COMPONENTS

$R F$ Sources for High-Gradient Accelerator Research

MODIFIED MAGNICON FOR HIGH-GRADIENT ACCELERATOR R\&D

\section{Cavity length}

If the cavity length changes, but some means to keep the same value of $Q$-factor is available, then it is advantageous to have a longer cavity. An example of this is given in Fig. 46. In the example, the $Q$-factor is assumed to be the same as the length increases; one observes little change in the efficiency; however, the field strength drops significantly, thus the probability of breakdown events drops as well. In reality, when the length increases, the amount of stored energy increases, and consequently the $Q$-factor goes up, which amounts to having larger fields. Practically, it is very challenging to maintain a $Q$-factor of 200 or less, when in the two-chamber configuration [see Fig. 44] the length of the $1^{\text {st }}$ chamber increases above $2.2 \mathrm{~cm}$. Thus, a practical choice is to strive to design a two-chamber configuration with the $1^{\text {st }}$ chamber having length $\sim 2.2 \mathrm{~cm}$; if a smaller length is needed [e.g. to fight against the spectrum of unwanted, parasitic modes], the $Q$-factor must be lowered below 200 .
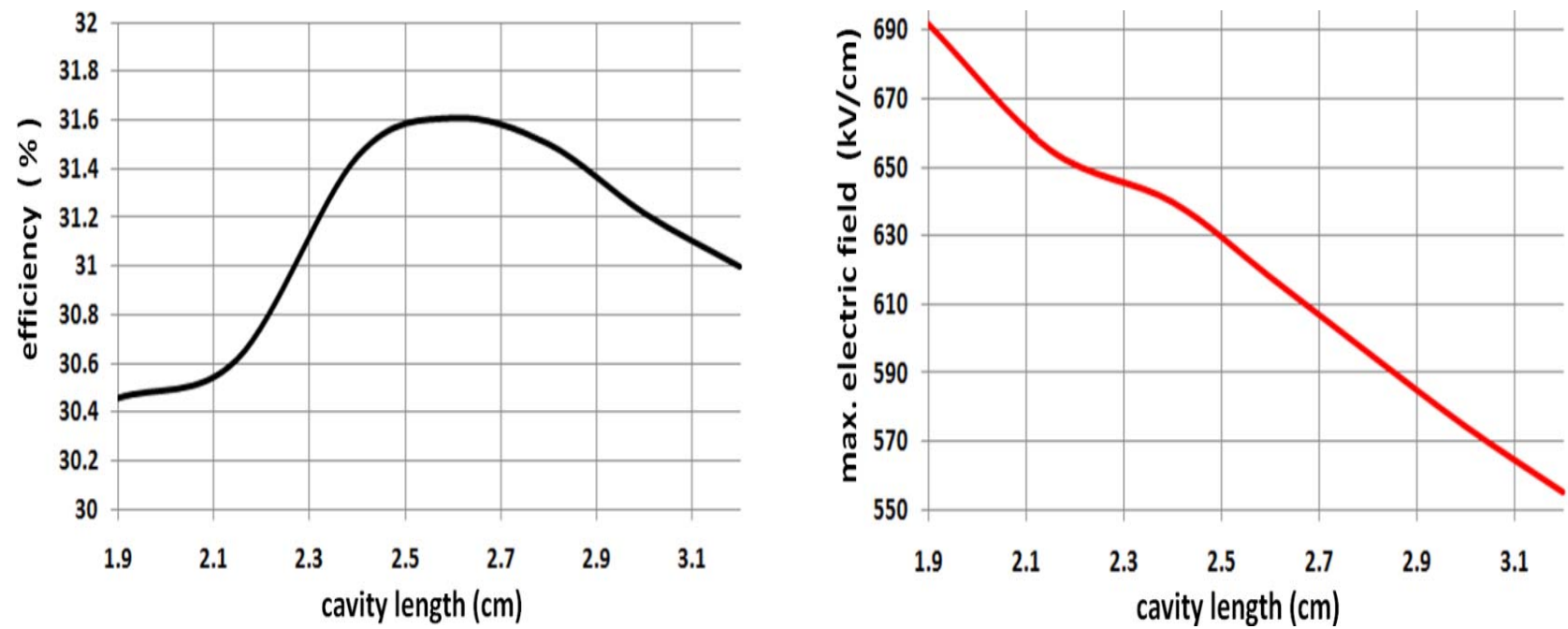

Fig. 46. Efficiency and maximum electric field $v s$ cavity length. This example is for a simple pillbox cavity with two drift tubes having radii of $4.25 \mathrm{~mm}$; the $Q$-factor is assumed to be the same (200) as the length varies. (Note that the curves may appear piece-wise because the simulation was done at discrete points, which are connected on these plots by lines for the sake of the narrative. 


\begin{abstract}
Omega-P, Inc.
Phase II proposal submitted following Phase I DoE SBIR grant DE-SC000 4497

in response to DoE SBIR/STTR 2010 Solicitation

Topic 65c: RADIO FREQUENCY POWER SOURCES AND COMPONENTS

$R F$ Sources for High-Gradient Accelerator Research

MODIFIED MAGNICON FOR HIGH-GRADIENT ACCELERATOR R\&D
\end{abstract}

\title{
III. CONCLUSIONS
}

All tasks of Phase I Project have been addressed and accomplished; the list follows:

Task 1: The first output cavity chamber will be optimized further. The second chamber will be designed and optimized, - is addressed in sub-section IIb.

Task 2: The possibility of using a new penultimate cavity (last deflection cavity) with the increased output aperture to allow large driver powers, and better efficiency will be investigated, taking into account that the spectrum of parasitic modes may change and destroy the beam dynamics, - is addressed in sub-section IIc.

Task 3: The possibility of an iron ring-like screen (made out of between the deflection cavity chain and the output cavity to prevent the magnetic flux exchange, and thus, guarantee the independence of electron beam dynamics in the deflection cavity chain from the magnetic fields created by the coils around the output cavity and vice versa will be investigated. That should insure more flexibility in optimization. The steps of task \#1 and 2 will be repeated, and a decision made whether the screen is worth implementing, - is addressed in sub-section IId.

Task 4: Simulations will be carried out to eliminate any parasitic modes in the entire output cavity near $34.3 \mathrm{GHz}$; that will require a periodic repetition of the steps from tasks \#1 and 2 to insure optimal cavity shapes for power production, extraction as well as for having the electric fields not exceeding desired limits, - is addressed in sub-section IIb.

Task 5: Simulations will be carried out to eliminate any modes with azimuthal number 3 whose frequencies are even as far from $34.3 \mathrm{GHz}$ as by $+/-1.5 \mathrm{GHz}$ in the output cavity; it is a well established fact, that such modes may couple themselves with the electron beam in the presence of the working mode - the so-called parametric excitation - and cause substantial deterioration of performances, - is addressed in sub-section IIb.

Task 6: A new collector will be designed, without ceramic insertions to have capability to braze it to the output cavity, - is addressed in sub-section IIe.

Task 7: Simulations will be carried out on the assembly consisting of the deflection cavity chain, two-chamber output cavity and the collector keeping in mind objectives stated in tasks \# 4 and $\# 5,-$ is addressed in sub-sections IIb and IIc.

Task 8: A mockup of the output cavity (from e.g. aluminum) will be manufactured, and its mode spectrum will be measured, as well as the Q-factor and frequency of the working mode - that is TE311-mode in the first chamber and TM310-mode in the second chamber, - is addressed in sub-section IIb. 


\section{Omega-P, Inc. \\ Phase II proposal submitted following Phase I DoE SBIR grant DE-SC000 4497 \\ in response to DoE SBIR/STTR 2010 Solicitation \\ Topic 65c: RADIO FREQUENCY POWER SOURCES AND COMPONENTS \\ RF Sources for High-Gradient Accelerator Research \\ MODIFIED MAGNICON FOR HIGH-GRADIENT ACCELERATOR R\&D}

\section{References}

1. V.P. Yakovlev, et.al, $100 \mathrm{MW}$ Electron Gun for a $34.3 \mathrm{GHz}$ Magnicon, Proceedings of the 2001 Particle Accelerator Conference (Chicago, IL 2001) p.1041.

2. O. A. Nezhevenko, et.al, $34 \mathrm{GHz}, 45 \mathrm{MW}$ Pulsed Magnicon, 10th Workshop on Advanced Accelerator Concepts-Mandalay Beach, CA 2002, AIP Conf. Proc. 647, 433 (2002).

3. O.A. Nezhevenko, et.al. High Power Millimeter- and Centimeter-Wave Magnicons for Particle Accelerator Applications, Proc. $7^{\text {th }}$ Workshop on High Energy Density and High Power RF, AIP Conf. Proc. 807 (2006) pp, 146-157.

4. O.A. Ivanov, et. al., Active Quasi-Optical Ka-band RF Pulse Compressor Switched by a Diffraction Grating, Phys. Rev. ST Accel. Beams 12093501 (2009).

5. M.A. LaPointe, et. al. First Experiments at the Yale University Ka-band Test Facility, Proc. $13^{\text {th }}$ Adv. Accel. Concepts Workshop, Santa Cruz, CA 27 July-2 August, 2008, AIP Conf. Proc. 1086 (2009) pp 470-476.

6. A.W. Fliflet and S.H. Gold, Mode Competition in Fourth-Harmonic Magnicon Amplifiers, IEEE Trans. on Plasma Science, 24 (1996) pp 957-963.

7. Y. Jiang, A. Vikharev, M. A. LaPointe, and J. L. Hirshfield, "CVD Diamond RF Breakdown Experiment", AIP Conf. Proc. 1299, 297 (2010)

8. P Slocum, et.al, "Search for 0.1 meV Axions and Hidden Photons Using Cu Resonant Cavities," Proceedings of Patras Workshop 2009, Patras, Greece (to be published) 